LBL- -25889

DE89 000273

\title{
The Production and Characterization of Mid-Gap States in trans-Polyacetylene
}

\author{
Carolyn Franklin Hoener \\ (Ph.D. Thesis) \\ Chemical Biodynamics Division \\ Lawrence Berkeley Laboratory \\ 1 Cyclotron Road \\ Berkeley, California 94720
}

August 1988

DISCLAIMER

\begin{abstract}
This report was prepared as an account of work sponsored by an agency of the United States Government. Neither the United States Governmeni nor any agency thereof, nor any of their employees, makes any warranty, express or implied, or assumes any legal liability or responsibility for the accuracy, completeness, or usefulness of any information, apparatus, product, or process disclosed, or represents that its use would not infringe privately owned rights. Reference herein to any specific commercial product, process, or service by trade name, trademark. manufacturer, or otherwise does not necessarily constitute or imply its endorsement, recommendation, or favoring by the United States Government or any agency thereof. The views and opinions of authors expressed herein do not necessarily state or reflect those of the United States Government or any agency thereof.
\end{abstract}

The United States Department of Energy has the right to use this thesis for any purpose whatsoever including the right to reproduce all or any part thereof. 
The Production and Characterization

of Mid-Gap States in trans-Polyacetylene

Copyright c 1988

Carolyn Franklin Hoener

The Goverment reserves for itself and others acting on its behalf a royalty free, nonexclusive, irrevocabie, world-wide license for govermental purposes to publish, distribute, translate, dupl icate, exhibit, and perform any such data copyrighted by the contractor. 


\author{
The Production and Characterization \\ of Mid-Gap States in traws-Polyacetylene
}

\title{
Carolyn Franklin Hoener
}

\begin{abstract}
1) Photoinduced IR absorptions with much longer lifetimes and lower excitation energies than previously reported were observed after the photolysis of polyacetylene with $v>5,000 \mathrm{~cm}^{-1}$. These absorptions are similar to those previously reported to have millisecond lifetimes, and are attributed to charged bond-alternation defects. The persistent photoinduced absorptions could be depleted by photoexcitation of a transition with an absorption maximum ne:ir $4,000 \mathrm{~cm}^{-1}$. Unpaired spins were observed to develop and be depleted at the same photolysis frequencies as the $\mathbb{R}$ activity. These spins may be due to the charged defects. This would mean that the defects are charged polarons. Two mechanisms, which use existing neutral bond-alternation defects as stepping stones, are proposed for the production of charged defects using mid-gap photons.
\end{abstract}

2) The temperature required to dope polyacetylene chemically was measured for various dopants. This was taken to be a measure of the activation energy for the doping reaction. The activation energies were found to be much lower than predicted by stepwise reaction mechanisms. Photolysis with above-band-gap light did not alter the ternperature required for reaction. The chemical stabilization of photoinduced defects, predicted to have a lower activation energy than the chemical doping reaction, does not occur at a perceptible rate at lower temperatures than the chemical doping reaction. This either implies that the activation energy for chemical doping is less than six kcal/mole, or supports the conclusion the conclusion that the photoinduced defects are actually polarons.

3) Attempts were made to photopolymerize acerylene, and various other molecules which could form polyacetylene and polyacetylene-like polymers, in the solid state. It was determined that light capable of photoexciting the monomers did penetrate the samples. Therefore it is believed that the monomer crystal structure inhibits solid-state polymerization. 


\section{Acknowledgments}

Too many peopie have contributed to my education and helped me through it for me to thank them all individually. My research director George C. Pimentel, Dr. Heinz Frei, and the members of their research groups have given me many helpful suggestions, criticisms and frequent assistance. The Department of Energy and various assorted loans have supported me financially, while society at large has been kind enough to tolerate my absence from the work force. My family, friends and teammates have been an essential source of moral support and momentary escape.

Sandra, however, needs to be thanked individually for almost always being helpful, almost always being right, never hesitating to point it out when I wasn't, and for all the cookies! 


\section{TABLE OF CONTENTS}

Page

\section{Acknowledgments}

Table of Contents

List of Figures

\section{Chapter I Introduction}

A Conducting Polymers 1

B.1 Preparation of Cuprene 1

B.2 Preparation of Linear Polyacttylene via Dehydrohalogenation 2

B.3.a Shirakawa Polymerization Method 2

B.3.b Alignment of Shirakawa Polyacetylene 2

B.4 Baker Polymerization Method 3

B.5 Precursors Routes 3

B.6 Narramann Polymerization Method 4

C.1 Chain Termination in Polyacetylene 4

C.2 Morphology of Polyacetylene 5

C.3 Average Polyacetylene Chain Length 5

C.4 Conformational Isomers of Polyacetylene 6

D.1 IR and Raman Spectra of Undoped Polyacetylene 9

D.2 Electronic Absorprions in Undoped Polyacetylene 10

D.3 Electrical Properties of Undoped Polyacetylene 13

D.4 Electron Paramagneric Resonance in Undoped Polyacetylene 13

D.5 Dynamic Nuclear Polarization in Undoped Polyacetylene 14

$\begin{array}{ll}\text { E.1 Doping Techniques } & 15\end{array}$

$\begin{array}{ll}\text { E.2 Compensation } & 15\end{array}$

E.3 IR and Raman Spectra of Doped Polyacetylene 15

E.4 Electronic Absorptions in Doped Polyacetylene 17

$\begin{array}{lll}\text { E.5 Electrical Properties of Doped Polyacetylene } & 17\end{array}$

E.6 Electron Paramagnetic Resonance in Doped Polyacetylene 17 
F. Exposure of Polyacetylene to Oxygen $\quad 18$

G.1 Photoinduced IR absorptions $\quad 18$

G.2 Photoinduced Electronic Absorptions 20

G.3 Electronic Properties of Photoexcited Polyacetylene 21

G.4 Electron Paramagnetic Resonance in Photoexcited Polyacetylene 21

H.1 Prototype molecules $\quad 22$

H.2 Conjugation and Delocalization in Prototype Molecules 24

I.1 Relationship Between Spin and Charge in Bond-Alternation Defects 27

I.2 History of Solitons 27

I.3 Mobility of Bond-Alternation Defects 28

I.4 Length of Bond-Altemation Defects 28

I.5 Electronic Structure of Bond-Altemation Defects 29

I.6 Vibronic Enhancement 31

I.7 The Pinning Mode 31

I.8 Formation of Bond-Alternation Defects 32

I.9 Photoproduction of Bond-Alternation Defects 33

I.10 Relationship Between Bond-Altemation Defects and Conductivity 34

J Science and technology 34

$\begin{array}{ll}\text { References } & 37\end{array}$

Chapter II Solid-State Acetylene 42

A. Solid-State Polymerization $\quad 42$

B. Acetylene Crystal Structures $4 \bar{Z}$

C. Excited States of Acetylene 44

D. Photochemistry of Acetylene 46

E.I Obtaining Crystalline Samples 46

E.2 Far-Uluraviolet Spectra of Acetylene 47

E.3 Attempts to Polymerize Solid Acetylene 50

E.4 Other Molecules 50

F. Search for Triplet Acetylene $\quad 52$

G. Insurmountable Difficulties 54

References $\quad 56$

Chapter III Experimental $\quad 58$

A Sample Preparation 58

$\begin{array}{lll}\text { A.1 Shirakawa Polymerization } & 58\end{array}$ 
A.2 Baker Polymerization 60

B.1 Sample Handling 61

$\begin{array}{lll}\text { B.2 Filters and Photolysis Setup } & 61\end{array}$

$\begin{array}{lll}\text { C.1 Conductivity Measurement } & 61\end{array}$

C.2 Photoconductive Response 63

$\begin{array}{lll}\text { D.1 } & \text { R setup } & 64\end{array}$

D.2 Doping at Low Temperature; IR and near-IR 65

D.3 Conductivity Measurements 65

D.4 Photoassisted Doping at Low Temperatures 66

E Long-Lived Photoinduced IR Activity 66

$\begin{array}{lll}\text { E.1 Isotope Shift } & 67\end{array}$

E.2.a Dependence of the Signal Intensity On Isomer Content 70

E.2.b Temperature Dependence of Signal Intensity 70

E.3 Dark Decay $\quad 70$

E.3.a Dependence of the Dark Decay Rate on Isomer Content 73

E.3.b Temperature Dependence of the Dark Decay Rate 73

E.4 Frequency Dependence $\quad 74$

E.4.a Depletion kinetics $\quad \cdot \quad 74$

E.4.b Growth Kinetics 82

E.5 Asymmetry $\quad 85$

E.6 Electronic Transitions 90

F.1 EPR Setup $\quad 90$

F.2 EPR Signal 91

$\begin{array}{ll}\text { References } & 94\end{array}$

Chapter IV Discussion 95

A Definitions 95

B.1 Chemically Induced Defects 96

B.2 Chemical Trapping of Photoinduced Defects 100

C.1 Observed Energy Levels in the tra.zs-Polyacetylene Band-Gap 105

C.2 Proposed Mechanisms for The Photoproduction of Transient Defects 108

D.1 Persistent Photoinduced Mid-Gap States 111

D.2 Defect Densities 111

D.3 Lifetime Discrepancies $\quad 112$

D.4 EPR Discrepancies $\quad 114$

$\begin{array}{lll}\text { D.5 Asymmetric Depletion } & 115\end{array}$ 
E.1 Fhotoproduction of Persistent Defects with Both Charge and Spin 117

E.2 Photodepletion of Persistent Defects 121

E.3 New Assignments For Mid-Gap States 121

F Persistent Photoinduced Mid-Gap Defects $\quad 122$

$\begin{array}{ll}\text { References } & 125\end{array}$

$\begin{array}{lll}\text { Appendix } 1 & \text { Thermodynamic Calculations } & 127\end{array}$

Appendix 2 Kinetic Calculations 131 
List of Figures

Figure

Page

Figure I-1 Configurational Isomers for Bond-Altemated Polyacetylene

Figure I-2 Catalyst Structure and Polymerization Process

Figure I.3 Prototype Molecules

Figure I-4 Absorption Maximum as a Function of Chain Length 25

Figure I-5 Predicted Energy Levels for Bond-Aisernation Defects 30

Figure II-1 The Low Temperature Crystal Structure of Acetylene

Figure II-2 Symmetry Considerations for Acetylene 45

Figure II-3 The Far-Ultraviolet Spectra of Solid $\mathrm{C}_{2} \mathrm{H}_{2}$ and $\mathrm{C}_{2} \mathrm{D}_{2}$

Figure II-4 Reaction Excitation Spectra 55

Figure IIl-1 Reaction Vessels 59

Figure III-2 Photcinduced IR Absorptions $\quad \cdot \quad 68$

Figure III-3 Decay of IR Activity in the Dark 41

Figure III-4 x rotodepletion of Photoinduced IR Activity 77

$\begin{array}{lll}\text { Figure III-5 Growth of Photoinduced IR Activity } & 78\end{array}$

Figure III-6 Populations from the Five-Parameter Fit 80

$\begin{array}{lll}\text { Figure III-7 Rates from the Five-Parameter Fit } & 81\end{array}$

Figure III-8 83

Growth and Depletion of IR activity with Photolysis at 5,810 cn-1

$\begin{array}{lll}\text { Figure III-9 } & \text { Poor Fit } & 86\end{array}$

Figure III-10 Location of the $1369 \mathrm{~cm}-1$ Absorption 88

$\begin{array}{lll}\text { Figure III-11 Typical EPR Spectrum } & 92\end{array}$

$\begin{array}{lll}\text { Figure IV-1 } & P \text {-Type Charge Transfer Doping } & 98\end{array}$

Figure IV-2 101

P-Type Chemical Stablization of Photoinduced Charged Solitons

Figure IV-3

102

$P$-Type Chemical Stablization of Photoinduced Charged Polarons 
Figure IV-4 Observed Transitions in trans-polyacetylene

Figure IV-5 Cycle A

Figure IV-6 Cycle B 
List of Tables

Table

Page

Tabie I-1

Assignments of the Infrared Spectra of Polyacetylene and Polyacetylene-d

Table I-2.
Assignments of the Raman Spectra of Polyacetylene and Polyacetylene-d

Table I-3. Defect Absorptions in the IR

$\begin{array}{lll}\text { Table II-1 IR Spectra of Acetylene } & 48\end{array}$

Table II-2 Far-Ultraviolet Spectra of Acetylene and Deuterated Acetylene 51

Table III-1 Photolysis Conditions 62

Table III-2 Sample (\#2) Transmitrance and Photoconductive Response 64

Table III-3 Persistent Photoinduced Absorptions 69

Table III-4 Signal Decay in the Dark 72

Table III-5 Depletion Kinetics

Table III-6 Growth Kinetics 76

Table III-7 Five Parameter Fit for Depletion Kinetics 79

Table III-8 Effective Rate Constants 87

Table III-9 $1369 \mathrm{~cm}^{-1}$ Peak Location $\quad 89$ 


\section{Chapter 1 Introduction}

\section{A. Conducting Polymers}

Normally the electrical properties of polymers are considered only for applications which require good insulators. Many polymers are used for electrical insulation: polyethylene, poly(vinyl chloride), and Teflon are commonly used as insulating coatings on wires and cable; Teflon and Kevlar are used in circuit boards; and polystyrene, polycarbonate, and polyester are used as dielectrics in capacitors. There are, however, some polymers with considerably more interesting electrical properties.

Electrically insulating in their pure forms, polymers such as polyacetylene, polythiophene, poly(p-phenylene sulfide) and several others, most with backbone structures similar to that of polyacetylene ${ }^{1}$, are subject to chemical interactions with electron donors and acceptors which can cause their conductivity to increase by more than twelve orders of magnitude ${ }^{2}$. Changes of this proportion in a single material are practically unheard of for any other physical property. For polyacetylene this means that a piece of plastic which is electrically insulating, can be changed into a material which is nearly as conductive as copper and several times less dense.

This material, however, does not conduct electricity in the same manner that copper does. When normal metals are cooled their conductivity increases, because the metal nuclei vibrate less and thus interfere less with the flow of electrons. When highly conductive doped pol;'acetylene (polyacetylene which has been exposed to chemical dopants, electron donors or acceptors) is cooled its conductivity decreases slightly3.

How, then, do doped polymers conduct electricity? What changes take place in these polymers when they are doped? Why do these changes take place? How do these changes cause the polymers to conduct? What are the charge carriers? A large amount of research has been devoted to these questions; the answers are not yet certain.

Because polyacetylene is the simplest polymer which can be made to conduct electricity, "the prototype corducting polymer", it has been the subject of most of the studies which address these questions. The studies herein were designed to provide new information about the nature and behavior of the charge carriers in polyacetylene. First, some of the earlier work on polyacetylene and its electrical properties is summarized.

\section{B.1 Preparation of Cuprene}

The first polymerization of acetylene was carried out under pressure over nickel acetlyacetone in tetrahydrofuran by $\operatorname{Reppe}^{4}$. This procedure makes a powdered polymer known as cuprene, because of its copper-like color. Unlike the polyacetylene of interest 
today, cuprene contains many aromatic, saturated and cross-linked regions, has low crystallinity, and cannot be doped to a conductive state 5 .

Other polymerization methods which produce saturated, cross-linked, and benzenoid polymers include the irradiation of solid or liquid acetylene with radon $\alpha$-rays ${ }^{6}$, cobalt $60 \gamma$-rays 7 , and mercury photosensitization of gas phase acetylene ${ }^{8}$. Similar material was also prepared by Hatano 5 using a triethylaluminum-titanium tetrachloride catalyst. This cataiyst is the archetype Ziegler-Natta catalyst, used for the production of stereospecific polymers from $\alpha$-olefins. Triethylaluminum-titanium tetrachloride is, however, not ideal for the polymerization of acetylene, because metal halides are capable of oxidizing polyacetylene 9 .

\section{B.2 Preparation of Linear Polyacetylene via Dehydrohalogenation}

Linear trans-polyacetylene was first produced by the dehydrohalogenation of poly(vinyl chloride) by Tsuchida and co-workers ${ }^{10}$. This process was carried out by dissolving poly(vinyl chloride) in tetrahydrofuran and adding sodium amide at room temperature. A black gel was produced, which dried to a powder with an electrical conductivity of $10^{-4} \Omega^{-1} \mathrm{~cm}^{-1}$.

\section{B.3.a Shirakawa Polymerization Method}

The first major breakthrough in polyacetylene research came in 1974 when Shirakawa and co-workers synthesized free standing films of polyacetylene ${ }^{11}$. A concentrated solution of triethylaluminum-titanium tetrabutoxide ( $3 \mathrm{mM}$ in Ti, $12 \mathrm{mM}$ in Al) in toluene is frozen to the walls of the reaction vessel at $195 \mathrm{~K}$. Acetylene is introduced to the reaction vessel, and polymerized as a thin film of cis-polyacetylene on the walls. The catalyst is washed away with pentane. The cis sample can be thermally isomerized to trans. The physical properties of polyacetylene prepared in this fashion, which will be described in detail in Chapter I.C - I.G, are a testament to the high quality of these films.

Undoped cis- and trans-Shirakawa polyacetylene have conductivities of $10^{-10}$ and $10^{-5} \Omega^{-1} \mathrm{~cm}^{-1}$, respectively. Doped samples can have conductivities $>10^{3} \Omega^{-1} \mathrm{~cm}^{-1}$.

\section{B.3.b Alignment of Shirakawa Polyacetylene}

A more ordered sample can be obtained by mechanically suretching the films obtained by the Shirakawa method. Cis- polyacetylene can be deformed by stretching it to more than three times its original length. This process partially aligns the fibrils along the stretching axis 12 . 
A greater fibril alignment can also be obtained by dissolving the catalyst in a nematic liquid crystal solution. The solution $i s$ allowed to flow down the reaction vessel walls causing maximum orientation of the liquid crystal. When acetylene is introduced it polymerizes into fibrils oriented along the axis of the liquid crystal, parallel to the direction of flow ${ }^{13}$.

Various other modifications have been made to the Shirakawa synthesis in attempts to improve the quality of the polymer with respect to one or another of its physical properties, typically at the expense of another property.

\section{B.4 Baker Polymerazation Method}

All linear polyacetylene samples contain some defect regions with unpaired spins. G. L. Baker's synthesis reduces the number of neutral defects (spins) in undoped transpolyacetylene from 1 per 3,000 carbon atoms, as in Shirakawa polyacetylene, to 1 per 47,000 carbon atoms. This is accomplished through the elimination of the steric constraints involved in solid-state cis-trans isomerization by allowing the isomerization to take place in solution, using a dilute catalyst solution, and carrying out the polymerization at room temperature. The catalyst solution, $0.05 \mathrm{M}$ in both triethylaluminum and ritanium tetrabutoxide, was mixed, with constant stirring, in the presence of acetylene. This resulted in a fine susper.sion of $-95 \%$ trans-polyacetylene. The suspension could be dried to a thin film of tiny fibrilous particles. Unfortunately these films are neither free-standing nor well suited to conductivity measurements ${ }^{14}$.

\section{B.5 Precursors Routes}

A completely different synthesis of polyacetylene was developed at Durham University. A soluble precursor polymer, poly-7,8-bis-(trifluoromethyl)-tricyclo[4.2.2.0]-deca-3,7,9-triene, was prepared by a catalyzed ring opening metathetical polymerization. Heat treament of this polymer results in the elimination of 1,2-bis(trifluoromethyl)benzene and isomerization of the remaining polymer to transpolyacetylene ${ }^{15}$. Because the precursor polymer is soluble it can be cast in various forms or drawn to single fibers ${ }^{16}$. Unidirecrional stress during heat treatment results in a highly crystalline polymer in which the polymer chains are believed to be oriented along the stretching axis ${ }^{17}$.

These polymers are very dense, $\rho=1.06 \mathrm{~g} \mathrm{~cm}^{-3}$, and do not have a fibril morphology. Undoped samples have 1 spin per $2,000 \mathrm{C}-\mathrm{H}$ units ${ }^{18}$. Despite average molecular weights of $200,000-400,000 \mathrm{amu}^{19}$, polyacetylene prepared in this manner has shorter conjugation lengths than that prepared by the Shirakawa method. High Raman 
frequencies and a larger band gap, 18,500 vs. $15,700 \mathrm{~cm}^{-1}$, indicate shorter conjugation lengths ${ }^{18}$. Unfortunately high conductivities have not been obtained in these samples, the doping process in these is significantly inhibited by the dense morphology and short conjugation lengths may also play a role.

More recently, a second precursor route, which does not involve the extrusion of molecular fragments, has been reported 20 . The ring opening metathesis polymerization of benzvalene produces polybenzvalene. This soluble polyacetylene precursor must be handled in solvents to minimize its tendency to cross-link. It can be converted to polyacetylene via thermal, photochemical, or must successfully, transition metal catalyzed isomerization. These polyacetylene samples are less desirable than samples prepared by the Shirakawa method because they have shorter conjugation lengths, lower crystallinity, and obtain lower conductivity on doping.

\section{B.6 Narramann Polymerization Method}

Narramann ${ }^{21}$ was able to reduce the number of $\mathrm{sp}^{3}$ defects in free standing polyacetylene films to a level below the detection limit of ${ }^{13} \mathrm{C}$ NMR with a modified Shirakawa polymerization. The catalyst solvent was replaced by silicon oil to allow for work at higher temperatures, allowing the catalyst to be prepared at $393 \mathrm{~K}$ and the polymerization to be performed at room temperature. The resultant polyacetylene film was $50 \%$ trans and could be suretch-deformed to six times its original length. Doped samples prepared in this manrer have the highest conductivities reported to date, as high as $1.5 \mathrm{x}$ $10^{5} \Omega^{-1} \mathrm{~cm}^{-1} .22$

\section{C.1 Chain Termination in Polyacetylene}

The polymerization of polyacetylene is almost always performed with the aid of a catalyst; in most cases the Ziegler-Natta catalyst triethylaluminum-titanium tertabutoxide is used. Whenever Ziegler-Natta catalysts are employed, the terminal end group of each polymer chain is either an $\mathrm{Al}$ or Ti organometallic group (-Al(Et) $)_{2}$ or $\left.-\mathrm{Ti}(\mathrm{OBu})_{3}\right)$. Elemental analysis shows trace amounts $(-0.1 \%)$ of $\mathrm{Al}$ and $\mathrm{Ti}$ in polyacetylene ${ }^{23}$. The terminal metal groups can be replaced with hydrogen atoms by alcoholysis or by treatment with methanolic $\mathrm{HCl}$. However, these procedures are seldom used on polyacetylene because of the risk of inadvertent doping 24 .

End groups are not believed to have much effect on the electrical properties of polyacerylene. The electrical behavior of interest is associated with conjugated trans segments. The Raman frequencies of conjugated polyenes depend on their length. This dependence has been used to determine ${ }^{25}$ that on average the trans segments in 
polyacetylene are 30 carbons long. A 400,000 amu molecular weight polymer would have 1,000 such segments only two of which cculd end with a polymer terminus. The other trans segments are separated by cis segments or $\mathrm{sp}^{3}$ defects, which allow the polymer to double back on itself. Since only $0.2 \%$ of the trans segments end with organometallic groups, these groups should have very little effect on the electrical properties of the bulk polymer.

\section{C.2 Morphology of Polyacetylene}

Polyacetylene samples must be formed in the shape in which they are to be used. Polyacetylene cannot be cast from solution because it cannot be dissolved in any known solvent which does not cause it to decompose. It cannot be molded from the melt either, since polyacetylene cross-links and decomposes at lower temperatures than it melts. Fortunately polyacetylene can be, and most often is, polymerized directly in the form of a free-standing film, suitable for spectroscopic measurements. These thin films are comprised of a loose, tangled mat of fibrils in which only $1 / 3$ of the sample volume is actually occupied. 26 The fibrils are $200-500 \AA$ in diameter and are mostly ( $\sim 86 \%)$ comprised of crystallites. 27

Sirice polyacetylene is insoluble it is not possible to obtain more ordered or homogenerus samples by recrystallization. Order within the sample can be increased to some extent by stretching the films. Films with a high cis content can be stretched to three times their original length, thus aligning the fibrils along the stretching axis. When these films are doped their electrical conductivity is anisotropic ${ }^{28}$. The conductivity along the stretching axis of a doped sample, which was stretched to three times its initial length before doping, is nearly 10 times that of a similar but unstretched sample (2800 vs. 300 $\left.\Omega^{-1} \mathrm{~cm}^{-1}\right)$. This gain in conductivity parallel to the stretching axis comes at the expense of the conductivity perpendicular to the stretching axis which is less than half the conductivity of the unstretched sample. The general conclusion from this result should be that conductivity is directed along the polymer fibrils, not that conductivity is directed along the polymer chain, as is sometimes implied. The polymer chains within the fibrils are not lined up in a bundle; they interweave and fold back on themselves in randomly oriented crystallites.

\section{C.3 Average Polyacetylene Chain Length}

Insolubility also means that light scattering, viscosity and various other techniques normally used to determine the average molecular weight and molecular weight distribution of polymers can not be used on polyacetylene. The average molecular weight cannot be 
determined by end-group analysis using ( $\left.{ }^{13} 1_{1}\right)$ iodine radioquenching because iodine can dope the polymer. It is, however, possible to determine the average moiecular weight by radioquenching techniques if tritiated methanol is used to replace the terminal $\mathrm{Al}$ and $\mathrm{Ti}$ groups on each chain with a tritium atom. 29 The conductivities observed for undoped samples after treatment with tritiated methanol are in the same range as those observed for untreated samples. 30 This suggests that the alcoholysis was carried out under conditions where the possibility of tritium doping was avoided. If the sample were doped with tritium, each chain would incorporate more than one ritium atom. Thus tritium radioquenching would give inaccurarely low molecular weights.

Tritium radioquenching has been used to determine the effects of polymerization temperature, catalyst aging time, monomer pressure, and $\mathrm{Al} / \mathrm{Ti}$ ratio on the average molecular weight. A maximum average molecular weight of $-900,000 \mathrm{amu}$ can be obtained with a polymerization temperature of $263 \mathrm{~K}$, monomer pressure of 760 torr, and a catalyst with a $4: 1$ Al : Ti ratio, $1 \mathrm{mM}$ in Ti, aged for $1 \mathrm{hr} .29,31$

Schen et $a l^{30}$ have looked at the conductivity of doped and undoped polyacetylene samples, and the thermopower of doped polyacetylene samples with average molecular weights ranging between 400 and $870,000 \mathrm{amu}$. They observed no significant or systematic dependence on molecular weight. From this they concluded that the motion of charge carriers is not confined to the chain and that intrachain movement is not the limiting source of resistance.

\section{C.4 Conformational Isomers of Polyacetylene}

Linear polyacetylene is made from acetylene by breaking one of the three bonds joining the carbon atoms in acetylene and to form single bonds between many such monomers. The resultant polymer is a long chain of carbon atoms alternately joined by double and single bonds, with one hydrogen attached to each carbon. This bonding scheme allows for four possible conformational isomers: trans-transoid, cis-transoid, transcisoid, and cis-cisoid (which is sterically unlikely) (Figure [-1). There are also non-bondalternating isomers, with the same geometries as the bond-aiternating isomers, in which all bonds are equivalent.

Crystallographic studies suggest that the cis isomer of polyacetylene observed is cis-transoid not trans-cisoid. X-ray scattering data from cis-polyacetylene matches patterns predicted for the cis-transoid structure more closely than those predicted for trans-cisoid. ${ }^{32}$ While this apparent agreement between theory and experiment may seem heartening it is worth noting that the proposed mechanism for the polymerization of acetylene by a ZieglerNatta catalyst, (Figure I-2) only allows for the production of the cis-transoid isomer. 33 

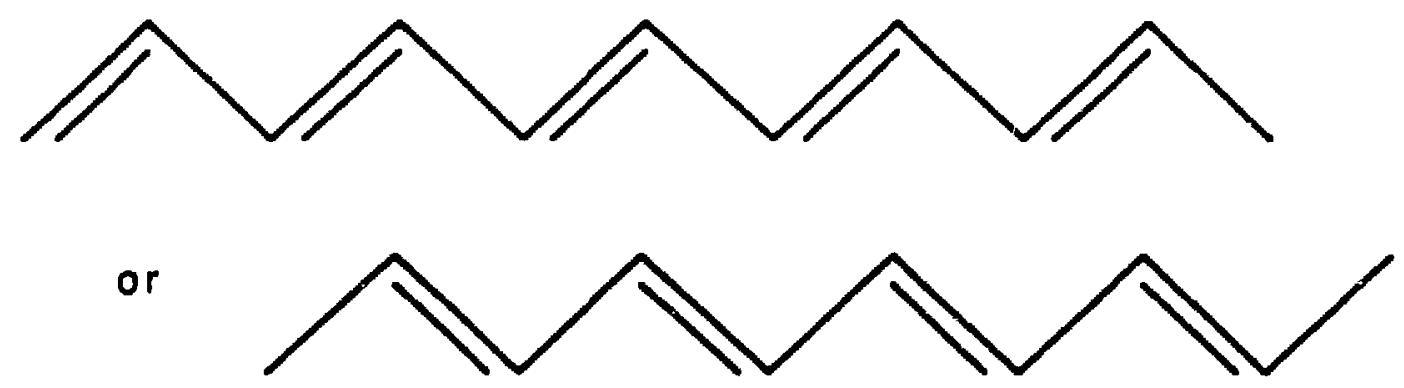

(A)

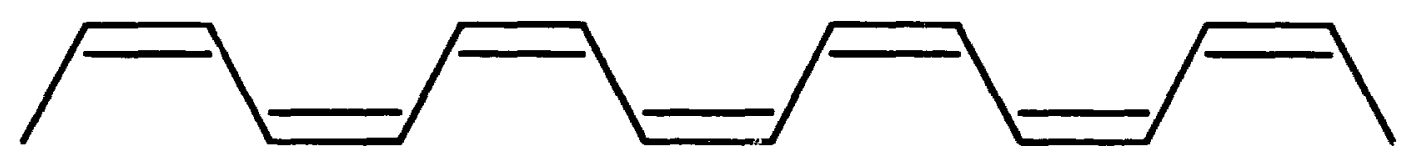

(B)

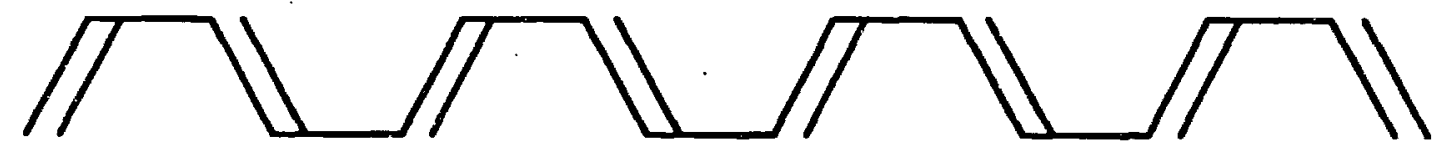

(C)

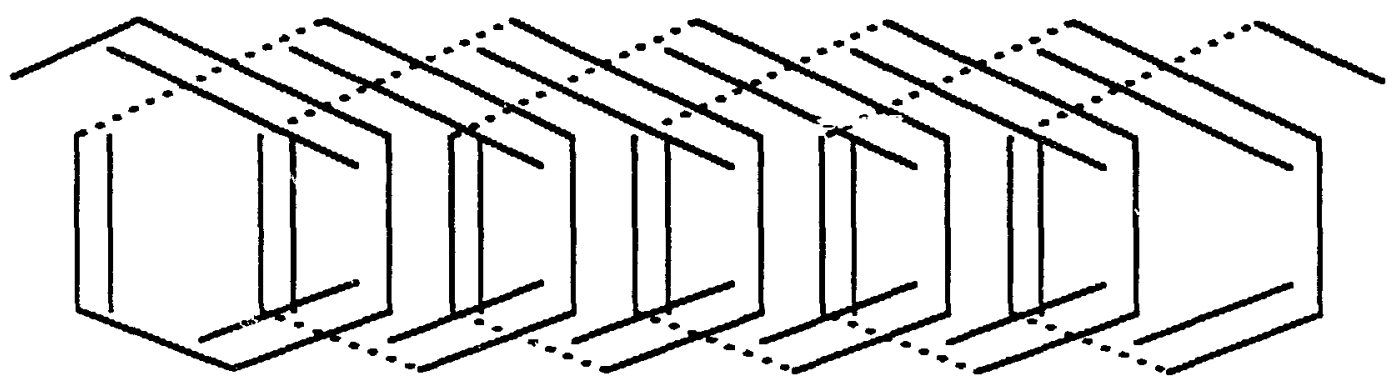

(D)

Figure I.1 Configurational Isomers for Bond-Altemated Polyacetylene

A) Two energetically degenerate forms of the trans-transoid isomer B) cis-transoid structure C) trans-cisoid structure D) cis-cisoid structure (sterically unlikely) 

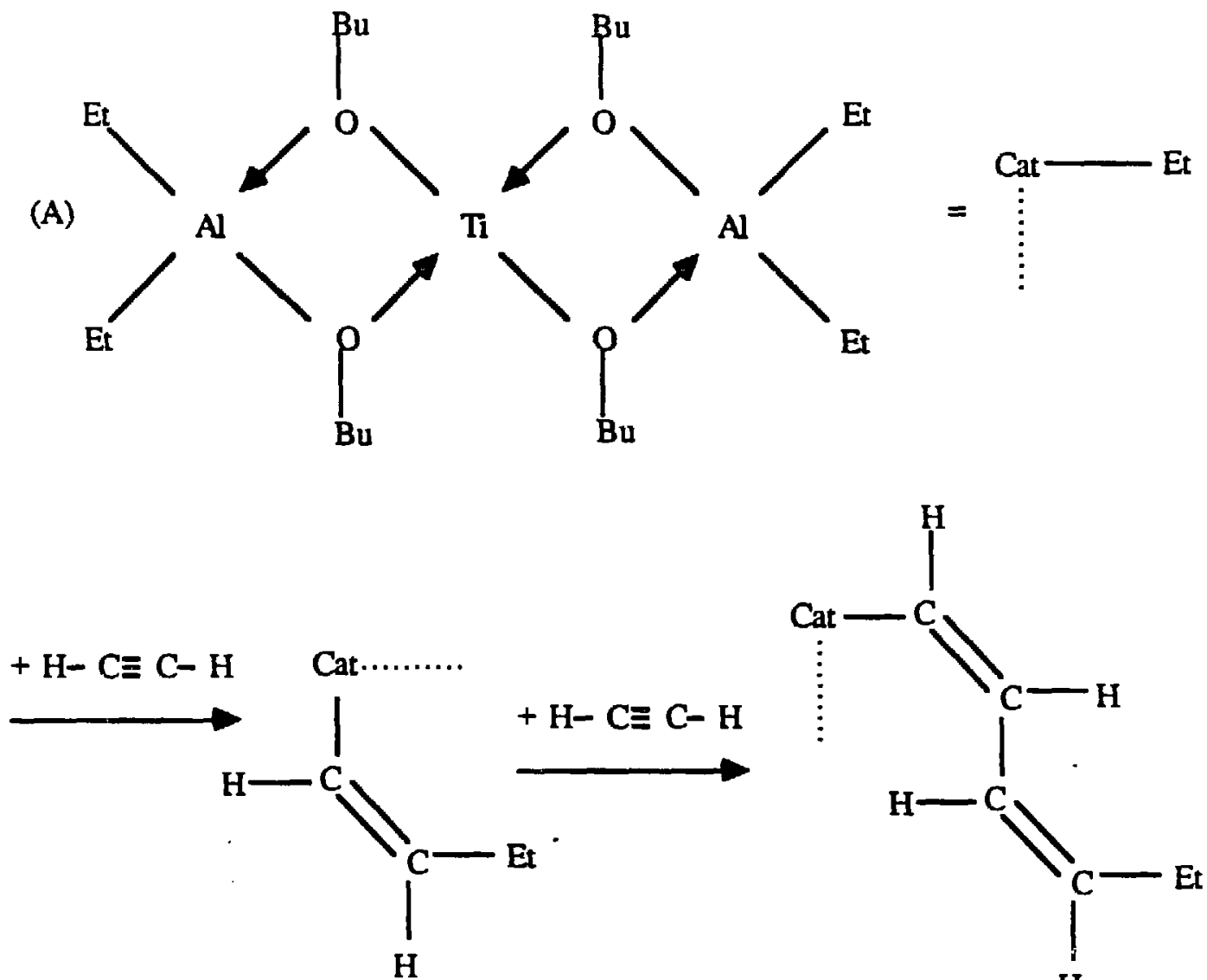

(B)
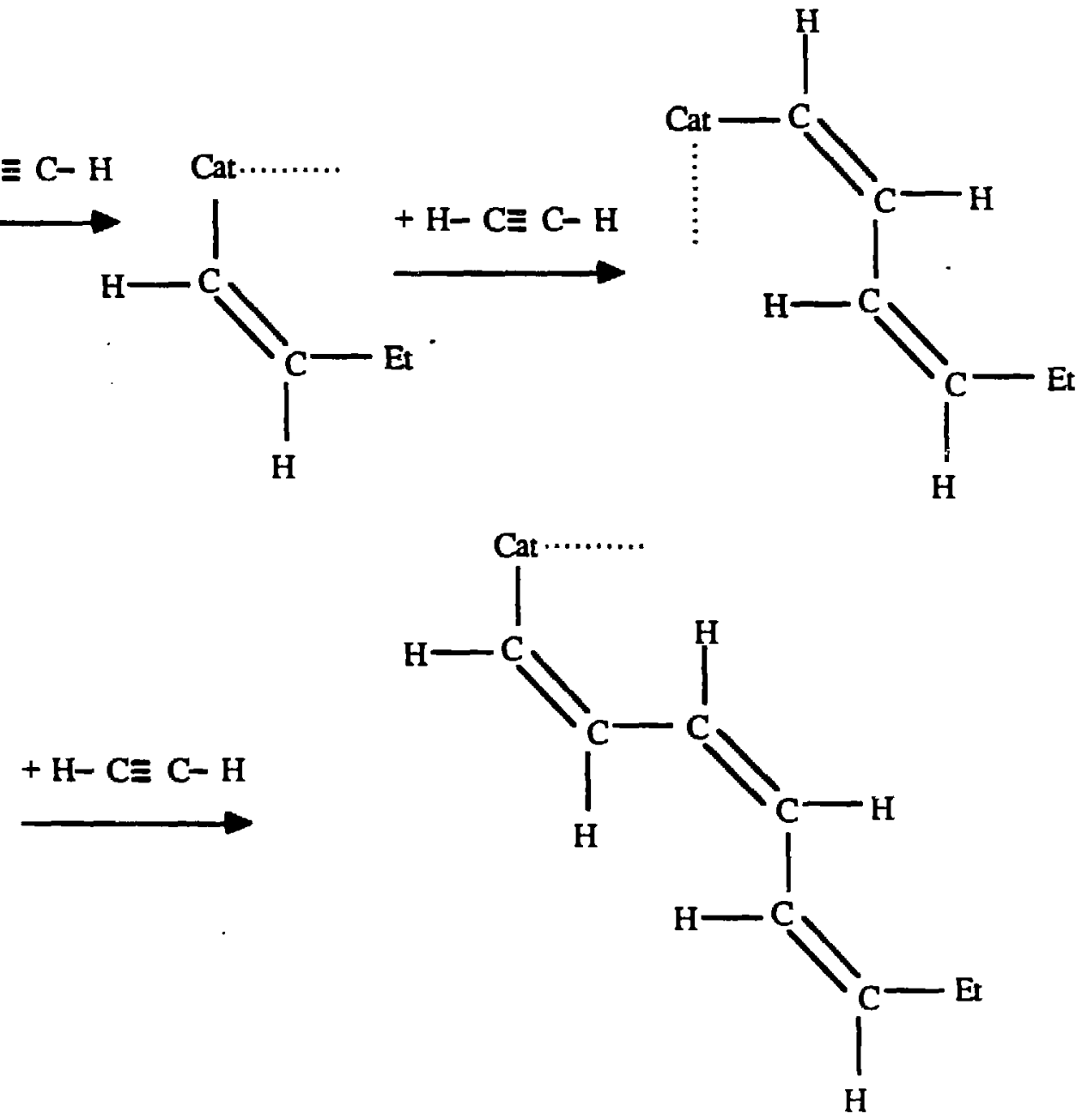

Figure I.2 Catalyst Structure and Polymerization Process
A) A proposed catalyst structure ${ }^{102}$
B) The proposed polymerization scheme 33 
The trans-transoid (trans for short) isomer has two forms which are mirror images of each other, and therefore energetically degenerate. The polymer can be found in either of these forms without preference. This degeneracy is central to the formation of soliton defects, discussed later.

Trans-polyacetylene is the most thermodynamically stable isomer. This is seen experimentally when the cis isomer is converted to trans upon thermal annealing. This is also in agreement with theoretical calculations. Similar calculations predict that the cistransoid isomer is more stable than the trans-cisoid isomer. Perego et al. ${ }^{32}$ fit their $\mathrm{X}$-ray scattering data on stretch-oriented polyacetylene to a model in which the symmetry breaking distortion parameter, $u_{0}$, was set to $0.04 \AA$, corresponding to bond lengths alternating between $1.435 \AA$ and $1.355 \AA$ in trans. This is a noticeably smaller difference in bond lengths than they observed for the cis isomer, $1.45 \AA$ and $1.34 \AA .32$ Fincher et al. ${ }^{34}$ have fit their data for trans polyacetylene with $\mu_{0}=0.03 \AA$. While these authors express little confidence in their value for $u_{0}$ because of difficulties related to the fibril and polycrystalline nature of the sample, they are confident that the observed scatrering partems are comparible with a bond-alternated chain, and not with a chain in which all carboncarbon bond lengths are equal.

\section{D.1 IR and Raman Spectra of Undoped Polyacetylene}

The difference in thermodynamic stability between the isomers has assisted in the analysis of the infrared spectra of polyacetylene. 26 Shirakawa and Ikeda compared the spectra of $(\mathrm{CH})_{x}$ and $(\mathrm{CD})_{x}$ samples prepared at various temperatures between 173 and 453 $\mathrm{K}$ to the predicted spectra for five model structures: trans structures with and without bond altemation, cis-transoid, trans-cisoid, and non-bond-aitemating cis structures. The spectra of samples prepared at temperatures below $173 \mathrm{~K}$ were most similar to the spectrum predic'ed for the cis-transoid structure. The spectra of samples prepared at $453 \mathrm{~K}$ was tentatively assigned to the non-bond-altemating trans structure.

The assignment for trans-polyacetylene was changed to the bond-alternating structure when normal coordinate calculations were performed to determine the commonly applicable force constants for in-plane vibrations for trons polyenes with 4,6, 8, and $n$ carbons ${ }^{35}$. These studies were extended to include out-of-plane vibrations and densities of states to complete the reassignment of the $\mathbb{R}$ and Raman spectra of trans-polyacetylene. ${ }^{36}$ This latest assignment attributes the absorption at $740 \mathrm{~cm}^{-1}$, previously assigned to $\mathrm{C}-\mathrm{H}$ out-of-plane bending motion in cis-polyacetylene, to a Raman allowed $\mathrm{B}_{\mathbf{g}} \mathrm{C}$-H out-ofplane bend in trans segments trapped between cis segments. This assignment is particularly disturbing since the standard method, which is used in this work, for the 
determination of isomer content is to compare the absorption of this band to that of the $1015 \mathrm{~cm}^{-1}$ band due to $\mathrm{C}-\mathrm{H}$ out-of-plane bending in trans. It remains a fact that the intensity of the $740 \mathrm{~cm}^{-1}$ absorption, now assigned to the out-of-plane $\mathrm{C} \cdot \mathrm{H}$ bending of trans segments trapped between cis segments, does decrease on thermal isomerization; therefore, comparison of this band to the $1015 \mathrm{~cm}^{-1}$ band gives at least qualitative information about isomer content.

The original and revised assignments for the IR spectra of polyacetylere are given in Table I-1. The assignments of the IR spectra of cis and trans-polyacetylene are in agreement with the findings from crystallographic data that the low temperature isomer of polyacetylene is cis-transoid and the high temperature form is bond-alternated transpolyacetylene.

Takeuchi's assignments for the Raman vibrations of trans-polyacetylene and Lichtman's ${ }^{37}$ for cis are compiled in Table I-2; values in parenthesis are for extremely weak bands. The Raman intensities are much greater ior trans-polyacetylene than for cis, and dominate the spectrum even when trans segments are present in only a few percent. This suggests that there is more vibronic coupling, which leads to resonant enhancement, in trans. The Raman frequencies vary with excitation wavelength. This variation is greatest for the 1450 and $1070 \mathrm{~cm}^{-1}$ bands ( 70 and $50 \mathrm{~cm}^{-1}$ respectively), the $C=C$ stretching, and $\mathrm{C}-\mathrm{H}$ in-plane bend plus $\mathrm{C}$ - $\mathrm{C}$ stretching modes in trans-polyacetylene. ${ }^{25}$ These modes are strongly coupled to the electronic excitation of the band gap. The local band gap and the Raman frequericies decrease with increasing conjugation length. 38 Thus higher excitation energies excite shorter trans segments, which have higher Raman frequencies. There are two strong Raman bands in trans-polyacetylene and three in trans $(C D)_{x}$. Cis-polyacetylene has three strong Raman bands while deuterated cis-polyacetylene has only two. Mixed isotopic substitution studies show that this reversal takes place continuously. The C-C stretching mode in cis loses intensity and the $\mathrm{C}$ - $\mathrm{C}$ stretching mode in trans gains intensity with increasing deuteration. ${ }^{100}$ Thus the vibronic coupling to the $\mathrm{C}-\mathrm{C}$ stretching mode increases on deuteration in trans and decreases in cis.

\section{D.2 Electronic Absorptions in Undoped Polyacetylene}

The cis and trans isomers of polyacetylene have distinct electronic absorption spectra. The onset of the interband ransition in trans-polyacerylene is at $11,300 \mathrm{~cm}^{-1}$, and the maximum is at $15,700 \mathrm{~cm}^{-1} .39$ In cis, where the bond-length altemation is greater, there is a larger band gap, with the absorption onset at $13,000 \mathrm{~cm}^{-1}$ and the absorption maximum at $18,500 \mathrm{~cm}^{-1} .40$ A very weak shoulder has been resolved on the band-edge 
Table I-1 Assignments of the Infrared Spectra of Polyacetylene and Polyacetylene-d

\begin{tabular}{|c|c|c|c|}
\hline $\begin{array}{l}(\mathrm{CH}) \mathrm{x} \\
\left(\mathrm{cm}^{-1}\right)\end{array}$ & $\begin{array}{l}(C D)_{x} \\
\left(\mathrm{~cm}^{-1}\right)\end{array}$ & Assignment 26 & Assignment ${ }^{36}$ trans \\
\hline $3057(v w)$ & $2275(\mathrm{vw})$ & B lu C-H stretch cis & \\
\hline $3044(w)$ & $2255(\mathrm{~m})$ & B3u C-H suretch cis & \\
\hline $3013(\mathrm{~m})$ & $2231(\mathrm{~m})$ & B3u C-H stretch trans & \\
\hline $1910-1720$ & $1550-1348$ & & $A u+B g u 7+u 8$ \\
\hline $1800(\mathrm{~m})$ & & $1329+446$ & catalyst \\
\hline $1630-1468$ & $1550-1348$ & & Ag viC $=$ C siretch \\
\hline $1690\left(w^{\prime}\right)$ & & $1249+446$ & \\
\hline $1448(s)$ & & $1050+402$ & \\
\hline $1360(v w)$ & & $947+402$ & \\
\hline $1329(s)$ & & Blu C-H i-p cis & \\
\hline & $1050(s)$ & Blu C-H i-p cists & unass. \\
\hline 1292 & 1201 & & Ag v2 C-C stretch \\
\hline $1292(\mathrm{vw})$ & $916(v w)$ & Biu C-H i-p trans & \\
\hline $1249(w)$ & $947(w)$ & B3u C-H i-p cis & Bu v4 \\
\hline $1170-1082$ & 861 & & $\begin{array}{l}\text { Ag v3 C-C stretch plus } \\
\text { C-H i-p }\end{array}$ \\
\hline $1118(v w)$ & & & B3v C-C stretch cis \\
\hline $1015(v s)$ & 752 (vs) & B2u C-H o-p trans & $\begin{array}{l}\text { Au v7 } \mathrm{CH}=\mathrm{CH} \text { C-H O-p, in } \\
\text { phase }\end{array}$ \\
\hline 890 & 797 & & $\begin{array}{l}\text { Bg u8 } \quad \mathrm{CH}=\mathrm{CH} \text { C-H o-p, in } \\
\text { phase }\end{array}$ \\
\hline 740 (vs) & $548(\mathrm{vs})$ & B2u C-H o-p cis & $\begin{array}{l}\text { Bg v8 CH=CH C-H o-p, out of } \\
\text { phase }\end{array}$ \\
\hline $446(v s)$ & 402 (vs) & Blu C-C-C def. cis & \\
\hline
\end{tabular}


Table I-2 Assignments of the Raman Spectra of Polyacetylene and Polyacetylene-d (values in parenthesis are for very weak absorptions)

$(\mathrm{CH})_{\mathbf{X}} \quad(\mathrm{CD})_{\mathbf{X}} \quad$ shift $\quad$ Assignment

$\left(\mathrm{cm}^{-1}\right) \quad\left(\mathrm{cm}^{-1}\right)$

$\operatorname{trans}^{36}$

$1464 \quad 1357 \quad 1.08 \quad \mathrm{C}=\mathrm{C}$ stretcch

(1294) $1200 \quad 1.08 \quad$ C-C stretch

$1072 \quad 855 \quad 1.25 \quad \mathrm{C}$-H in-plane bend plus $\mathrm{C}-\mathrm{C}$ stretch

cis $^{37}$

$\begin{array}{rrrl}1540 & 1470 & 1.05 & \text { C=C stretch } \\ 1250 & 976 & 1.29 & \text { C-il in-plane bend plus C-C stretch } \\ 910 & (765) & 1.19 & \text { C-C stretch }\end{array}$


absciption of trans-polyacetylene with photoacoustic spectroscopy ${ }^{41}$ and in direct absorption spectroscopy on compensated samples ${ }^{42}$ near $12,000 \mathrm{~cm}^{-1}$.

Additional absorptions, in the band gap of trans-polyacetylene, have been reported. A band at $7,300 \mathrm{~cm}^{-1}$ has been observed by direct absorption ${ }^{43}$ and by photothermal deflection spectroscopy. 14 Suzuki et al..$^{43}$ attribute this band to neutral soliton defects (defined in Chapter I-I). They reason that since the band edge is at $11,300 \mathrm{~cm}^{-1}$, mid-gap states should be $5,600 \mathrm{~cm}^{-1}$ above the band edge or $7,300 \mathrm{~cm}^{-1}$ above the maximum density of states. Baker et al. ${ }^{14}$ attribute this band to charged soliton defects caused by inadvertent oxidation. The fact that photoacoustic studies on both cis and transpolyacetylene have failed to detect the $7,300 \mathrm{~cm}^{-1}$ band supports this assignment since the defect concentration would be determined by the sample preparation. Instead, a relatively sharp peak at $5,950 \mathrm{~cm}^{-1}$ was seen for both isomers in the photoacoustic studies. ${ }^{41}$ This was assigned as an overtone of the $\mathrm{C}$-H stretching mode.

\section{D.3 Electrical Properties of Undoped Polyacetylene}

The electrical conductivity of undoped trans-polyacetylene is roughly $10^{-5} \Omega^{-1} \mathrm{~cm}^{-1}$ and $10^{-10} \Omega^{-1} \mathrm{~cm}^{-1}$ for cis .44 The d.c. conductivity decreases with decreasing temperature . for both isomers. ${ }^{45}$ This is typical in large-gap semiconductors because fewer charge carriers are thermally generated at low temperanures. A straight line fit was obtained from a plot of $\log \left(\sigma_{\mathrm{d} . c .}\right)$ vs. $\mathrm{T}^{-1 / 4}$ for trans-polyacetylene. The temperature dependence of the a.c. conductivity in trans is very strong; it was not fit to an analytical form. The frequency dependence is proportional to $\mathrm{f} .7$ at temperatures above $200 \mathrm{~K}$, proportional to 0.5 below $200 \mathrm{~K}$.

\section{D.4 Electron Paramagnetic Resonance in Undoped Polyacetylene}

Evidence of radical defects in polyacetylene is seen in electron paramag:etic resonance (EPR) experiments. When acetylene is polymerized at low temperatures. without warming up or exposing to oxygen, there is no $g=2$ EPR signal. 46 When the sample is warmed spin defects are irreversibly produced. At room temperature a predominantly cis sample, prepared in the standard fashion, has 1 spin per $3,000 \mathrm{C}-\mathrm{H}$ units and a linewidth of $\Delta \mathrm{H}_{\mathrm{p}-\mathrm{p}}=8.5 \mathrm{G}$. A predominantly trans sample has I spin per 30,000 $\mathrm{C}-\mathrm{H}$ units and a linewidth of $\Delta \mathrm{H}_{\mathrm{p} \sim \mathrm{p}}=1.2 \mathrm{G}$. The EPR signals of both isomers are near $\mathrm{g}$ $=2$ and they obey the Curie law, i.e. increase in intensity with decreasing temperatures. Such signals are typical of organic free radicals. Power saturarion curves suggest that the signal for cis-polyacetylene is inhomogeneously broadened while the signal for trans is homogeneously broadened. 
The activation energy for the creation of spin defects, $E_{a}=10 \mathrm{kcal} / \mathrm{mol}$, has been derived from the temperature dependence of the rate of formation, and is independent of isomer content. ${ }^{47}$ This is noticeably smaller than the activation energy for cis-trans isomerization, $\mathrm{E}_{\mathrm{a}}=17-18 \mathrm{kcal} / \mathrm{mol}$, which increases with trans content. The log of the spin concentration increases linearly with the trans content.

The EPR signal linewidth has been attributed to hyperfine coupling. 48 The linewidth for cis- $(\mathrm{CH})_{\mathrm{x}}$, but not for $(\mathrm{CD})_{\mathrm{x}}$, increases at low temperatures. This suggests that spin defects on cis-polyacetylene are delocalized and immobile. The defect width has been determined from the linewidth and the well known hyperfine coupling constants for electrons localized on single $C-H$ and $C-D$ units. The defects are believed to extend $o^{\circ}$ or a distance of 6-12 C-H units. The EPR signal linewidths for both trans-(CH) $)_{x}$ and $(\mathrm{CD})_{\mathbf{x}}$ increase at low temperatures, which is interpreted to mean that the defects in transpolyacetylene are mobile.

Thomann and Baker ${ }^{49}$ have raised questions about this interpretation. They prepared a powdered sample of $95 \%$ trans-polyacetylene with approximately the same spia concentration as the cis films discussed above. The EPR signal for this sample was five times as broad as the signals observed for trans films. The ENDOR spectrum of this sample was also very reminiscent of the cis films. This would suggest a concentration effect on the EPR lineshape.

\section{D.5 Dynamic Nuclear Polarization in Undoped Polyacetylene}

Dynamic nuclear polarization (DNP) studies ${ }^{50}$ also indicate mobile spin defects in trans-polyacetylene and fixed spin defects in cis. Fixed spins give DNP signals with odd symmetry, due to the solid-state effect. The Overhauser effect causes signals with even symmetry for mobile spins. In a series of samples with varying isomer coutent, the Overhauser effect played a greater role in samples with higher trans content, giving symmetric DNP signals. The solid-state effect dominated in samples with a high cis content, giving nearly anti-symmetric DNP signals.

Temperature dependent DNP studies ${ }^{51}$ on trans-polyacetylene indicate that the spin defects, which are mobile at room temperature, become rapped at lower temperatures, 10 $\mathrm{K}<\mathrm{T}<100 \mathrm{~K}$. Below $30 \mathrm{~K}$ the solid-state effect is dominant, indicating fixed spins, while above this temperature the diffusion coefficient for spin defects, calculated from the proton relaxation rate, is proportional to $\mathrm{T}^{-1 / 2}$. Spin defects diffuse their length in $17 \mathrm{Fs}$ at $300 \mathrm{~K}$. 


\section{E.1 Doping Techniques}

The properties of polyacetylene are drastically altered by chemical doping. Chemical doping, the interaction between polyacetylene and an electron acceptor or donor, to produce charged defects in the band gap can be effected in a number of ways. Polyacetylene can be doped by exposure to vapors of electron acceptors such as $\mathrm{I}_{2}, \mathrm{Br}_{2}$, AsF $_{5 .}{ }^{28}$ The homogeneity and extent of doping can be controlled by the vapor pressure and exposure time. Solutions containing compounds which can chemically dope polyacetylene, such as $I_{2}$ or sodium naphthalide, can be used to dope polyacetylene. Solutions which contain compounds which cannot chemically dope polyacetylene can be used to dope polyacetylene electrochemically. ${ }^{39} \mathrm{~A}$ cell containing $\mathrm{Li}\left(\mathrm{ClO}_{4}\right)$ in which transpolyacetylene is used for both electrodes, can be driven by a potential of 4 volts. The polyacetylene anode is $p$-doped as it loses electrons to the battery, and forms a salt with the $\mathrm{ClO}_{4}-$ ion. When electrons from the battery flow into the polyacetylene cathode negatively charged defects are developed, which form salts with the $\mathrm{Li}^{+}$ions. These processes are completely reversible, and the electrochemical cell will discharge,acting as a battery, if the applied potential is removed. If cis-polyacetylene is used for the electrodes, it is irreversibly isomerized to trans during the first doping cycle. 52

\section{E.2 Compensation}

Chemical doping can also be reversed, compensated for, except for the cis-trans isomerization. ${ }^{29}$ Electrically conducting sodium $n$-doped polyacetylene loses its conductivity when exposed to $I_{2}$ vapors until there are an equal number of iodine and sodium atoms. After this point the polyacetylene can be $p$-doped by the $I_{2}$ vapors and regain its conductivity. Polyacetylene samples as they are prepared are slightly $n$-doped, as seen by their positive thermopower coefficient .30 Occasionally, undoped samples are compensated to their minimum conductivity, and then considered rigorously "undoped" samples. 48 A weak shoulder on the onset of the band gap absorption has been detected in $\mathrm{NH}_{3}$-compensated samples with photothermal deflection spectroscopy, 42 and is therefore not attributed to charged defects.

\section{E.3 IR and Raman Spectra of Doped Polyacetylene}

Doping causes dramatic changes in the IR spectrum of polyacetylene. ${ }^{53}$ Strong new IR absorptions develop at 1385,1295 , and $832 \mathrm{~cm}^{-1}$, when either isomer is doped. The $\mathrm{fu}_{\mathrm{i}}$, width half maximum (FWHM) of these absorptions are 104, 30, and $-400 \mathrm{~cm}^{-1}$ respectively. Only two strong bands, at 1088 and $697 \mathrm{~cm}^{-1}$ (FWHMs 158 and -400 $\mathrm{cm}^{-1}$ ), develop when deuterated polyacetylene is doped. The frequencies of the 1385 and 
$1295 \mathrm{~cm}^{-1}\left(1088 \mathrm{~cm}^{-1}\right.$ for $\left.(C D)_{x}\right)$ absorptions are very sample-dependent and may vary as much as $30 \mathrm{~cm}^{-1}$ with the same dopant. The $832 \mathrm{~cm}^{-1}\left(697 \mathrm{~cm}^{-1}\right)$ absorption is several hundred wavenumbers wide; therefore the frequency reported for its maximum is rather imprecise. Within these accuracies the location of these bands is nearly independent of the choice of dopant, and slightly dependent on the dopant concentration. 54 There is also a broad absorption in the near-IR, $-4500 \mathrm{~cm}^{-1}$, which develops upon doping of either isotope. This band is believed to be due to an electronic transition because of its shape. location and the lack of an isctope shift. 55

The dopant-induced mid- $\mathbb{R}$ spectrum is reminiscent of the Raman spectrum of trans-polyacetylene, in that there are three strong bands for $(\mathrm{CH})_{\mathbf{x}}$, and two strong bands for $(C D)_{\mathbf{x}}$. This similarity led to the early assignment of these bands to the Raman-allowed molecular vibrati is of trans-polyacetylene. 56 The presence of the dopant was believed to cause the selectic.s rules to break down. The intensity of these features was attributed to vibronic enhancement by the charge imparted to the chain during the charge transfer reaction with the dopant. However, on mixed isotope substitution, these bands do not behave the same as the Raman bands in trans-polyacetylene. ${ }^{100}$ This observation called for an altemate assignment. Reassignment was also in order because the defect $\mathbb{R}$ and the trans Raman vibrations are at significantly different frequencies and experience different isotope shifts.

The dopant-induced infrared active modes of polyacetylene at $1370\left(1088\right.$ in $\left.(C D)_{x}\right)$ and $1290 \mathrm{~cm}^{-1}$ are now believed to be due to molecular vibrations of localized, charged defects on the polymer chain, in which the bond alternation is lost.57 They are assigned to motions similar to the undoped trans Raman modes, coupled to phonon modes. This still does not account for the different isotope shifts. Again, vibronic enhancement from the charge on the defect is used to explain the intensity of these feaiures. The 890 (697 in $\left.(C D)_{x}\right)_{\mathrm{cm}^{-1}}$ absorption is associated with the movement of the defect along the chain, brought back to its initial position by the electrostatic force between the charged defect and the dopant counter-ion. It is known as the pinning mode. 58

Raman studies on lightly doped polyacerylene support this interpretation. 59 As discussed earlier, the frequencies of Raman absorptions in trans-polyacetylene are indicative of the conjugation lengths. When polyacetylene is lightly doped its Raman absorptions are shifted to lower frequencies, indicating a shortening in the uninterrupted lengths of the bond-altemating structure. This is consistent with the formation of defects which interrupt the backbone structure.

Rapid dopant-induced cis-trans isomerization can be observed with Raman spectroscopy during light doping of polyacerylene. ${ }^{60}$ The condition of the dopant species 
after doping has also been investigated using Raman. 38 The reduction of $\mathrm{I}_{2}$ to $\mathrm{I}_{3^{-}}$or $\mathrm{I}_{5}^{-}$ has been determined in this manner, supporting the belief that chemical doping involves a charge transfer reaction between the polymer and the dopant.

\section{E.4 Electronic Absorptions in Doped Polyacetylene}

When polyacetylene is doped the optical absorption of its interband transition (onset $11,300 \mathrm{~cm}^{-1}$, peak $15,700 \mathrm{~cm}^{-1}$ for trans; onset $13,000 \mathrm{~cm}^{-1}$, peak $18,500 \mathrm{~cm}^{-1}$ for cis) is suppressed, and a new band grows in at lower energy. 54 The peak absorption of this new band is at $\sim 7,000 \mathrm{~cm}^{-1}$ in lightly doped polyacetylene, and gradually shifts to $\sim 4,000 \mathrm{~cm}^{-1}$ in heavily doped samples. 55 This peak extends from below 300 to approximately 25,000 $\mathrm{cm}^{-1}$. Cis-polyacetylene can be doped with $\mathrm{BF}_{3}$ or $\mathrm{AsF}_{5}$, but not $\mathrm{I}_{2}$, to the point where the interband transition is lost altogether. No data is presented in which trans is doped to this extreme. The extent to which the interband transition can be suppressed seems to be a function of the dopant used, and may be a result of inhomogeneous doping. Inhomogeneous doping can be caused by the hindered diffusion of large dopant molecules or surface doping by highly reactive dopants.

\section{E.5 Electrical Properties of Doped Polyacetylene}

Chemical doping can increase the electrical conductivity of polyacetylene more than twelve orders of magnitude. D.c. conductivity measurements made with a four point probe show a dramatic increase for dopant concentrations, $\left(\mathrm{CHZ}_{\mathbf{y}}\right)_{\mathrm{x}}, \mathrm{y}<0.1 .61$ In this range log $\sigma_{\text {d.c. }}$ increases linearly with $\log y$. The mictowave conductivity at low dopant concentrations (e.g. $y=0.005$ ), is somewhat greater than the d.c. conductivity. As the dopant concentration increases the temperature dependence of the d.c. conductivity (log $\sigma_{\text {d.c. }} \alpha \mathrm{T}^{-1 / 4}$ ) is lost. ${ }^{3}$ Above $\mathrm{y}=0.1$, the conductivity is nearly temperature independent. Lightly $p$-doped polyacetylene, $y=0.0001$, has an activation energy for conduction, $E_{a}=$ $0.3 \mathrm{eV}$, and a large positive thermopower coefficient, $S=800 \mathrm{mV} / \mathrm{K}$. These both begin to drop when the dopant concentration reaches $\mathrm{y}=0.001$, and approach zero at $\mathrm{y}=0.01$.

\section{E.6 Electron Paramagnetic Resonance in Doped Polyacetylene}

The EPR signal of undoped polyacetylene has a Gaussian line shape and obeys the Curie law (intensity increases with decreasing temperature). These features are characteristic of spins localized in mid-gap states in semiconductors. ${ }^{62}$ When polyacetylene is doped the number of Curie law spins begins to drop at dopant concentration, $\left(\mathrm{CHZ}_{y}\right)_{x}, y=0.002$, and reaches zero by $y=0.04 .63,45$ In the same concentration range, an EPR signal with Dysonian line shape which exhibits Pauli law 
susceptibility (intensity is temperature-independent) grows in. Dysonian line shape and Pauli law susceptibility are characteristic of normal metals.

\section{F Exposure of Polyacetylene to Oxygen}

Molecular oxygen is a weak electron acceptor, and does not dope polyacetylene to a highly conducting state. ${ }^{64}$ There is a slight increase in conductivity when polyacetylene is first exposed to $\mathrm{O}_{2}$, but this is followed by a decrease to well below the original value. The drop in conductivity and degradation of the polymer begin immediately at temperatures above $350 \mathrm{~K}$. Exposure to $\mathrm{O}_{2}$, at room temperature, also causes a loss in conductivity for doped polyacetylene.

EPR studies indicate that exposure to $\mathrm{O}_{2}$ initiates the formation of spin defects in cis-polyacetylene. 46 When trans-polyacetylene is exposed to $\mathrm{O}_{2}$ the EPR power saturation curve is altered to indicate inhomogeneous broadening; this effect is reversible. 65 Raman studies have shown that cis-trans isomerization is facilitated by the presence of $\mathrm{O}_{2} .66$ It has also been shown that exposure to $\mathrm{O}_{2}$ partially destroys a sample's ability to be doped. Oxygen impurities result in infrared absorptions at 1665, 1720,1140, 887, and 3450 $\mathrm{cm}^{-1} .26$ These are attributed to ketones $\left(1665\right.$ and $\left.1720 \mathrm{~cm}^{-1}\right)$, peroxides and epoxides (1140 and $\left.887 \mathrm{~cm}^{-1}\right)$, and hydroxy groups $\left(3450 \mathrm{~cm}^{-1}\right)$. This is clear evidence that there are reactions between $\mathrm{O}_{2}$ and polyacetylene other than doping.

\section{G.1 Photoinduced IR absorptions}

Transient IR absorptions, similar to those which develop on chemical doping, are observed during the photolysis of trans-polyacetylene at photon energies higher than 9,000 $\mathrm{cm}^{-1} .67$ The spectra of the transient absorptions were recorded by ratioing the sample's spectra before and during excitation. A lifetime of $1 \mathrm{~ms}$ for these absorptions was determined from the dependence of the photoinduced absorption intensity on the chopping frequency of the excitation beam. 68 The intensity of similar transient absorptions observed in cis-polyacetylene is consistent with the percentage of trans isomer in the sample. Two additional weak absorptions, not seen in doped polyacerylene, have been resolved in the photoinduced spectrum. The frequencies of the photoinduced absorptions in transpolyacetylene ${ }^{69}$ are given in Table I-3.

The intensity of the photoinduced absorptions increases as the square root of the excitation power, saturating at $50 \mathrm{~mW} / \mathrm{cm}^{2} .68$ Assuming similar extinction coefficients for photo- and dopant-induced defects, the maximum concentration of photoinduced defects is approximately $5 \times 10^{17} \mathrm{~cm}^{-3}(-1 / 37,000 \mathrm{C}-\mathrm{H}$ units). The intensity of the photoinduced absorptions decreases slightly as the temperature of the sample is raised from 10 to $150 \mathrm{~K}$. 
Table I-3. Defect Absorptions in the IR

Experimental

\begin{tabular}{|c|c|c|c|c|c|}
\hline & (C & & & & \\
\hline & $\left(\mathrm{cm}^{-1}\right)$ & rel.int. & $\left(\mathrm{cm}^{-1}\right)$ & rel.int. & shift \\
\hline Dopant Induced & 1385 & & 1088 & & 1.27 \\
\hline & 1295 & & 1223 & weak & 1.06 \\
\hline & 832 & & 670 & & 1.24 \\
\hline Photoinduced & 1438 & 0.16 & 1340 & 0.7 & 1.07 \\
\hline & 1365 & 4.2 & 1045 & 21.0 & 1.31 \\
\hline & 1288 & 1.0 & 1227 & 1.0 & 1.05 \\
\hline & 1034 & 0.2 & 858 & 0.3 & 1.20 \\
\hline$\cdot$ & 536 & 29.0 & 510 & 41.0 & 1.05 \\
\hline
\end{tabular}

Theoretical 57

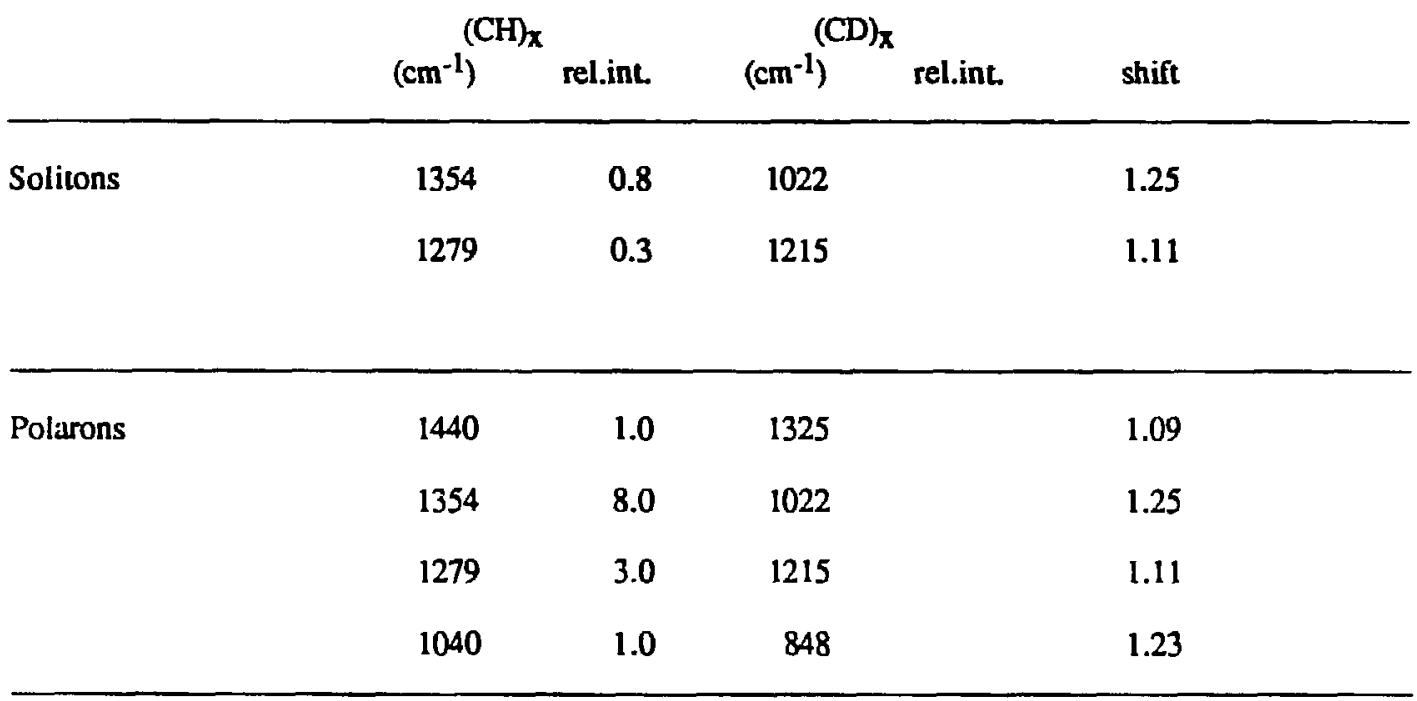


Above $150 \mathrm{~K}$ the intensity drops off dramatically. In this same temperature range ( $150 \mathrm{~K}$ and up) photoconductivity develops, and the gain increases with temperature. The excitation energy dependence for transient photoinduced IR absorptions and room temperature photoconductivity are the same, both increasing from $9,000 \mathrm{~cm}^{-1}$ to 12,000 $\mathrm{cm}^{-1} .70$

The photoinduced IR absorptions in lightly doped polyacetylene are slightly less intense than those in undoped polyacetylene. The intensity decreases with increasing dopant concentration. ${ }^{71}$ At a dopant level of $y=0.05$ the photoinduced absorption intensity suddenly decreases more rapidly. In lightly doped polyacetylene the transient absorption at $1360 \mathrm{~cm}^{-1}$ is accompanied by bleaching of the $1390 \mathrm{~cm}^{-1}$ dopant-induced defect absorption. ${ }^{72}$ The integrated absorption intensity for this region remains constant.

\section{G.2 Photoinduced Electronic Absorptions}

The transient effects observed in the electronic structure of trans-polyacetylene during photoexcitation above the band gap (above $12,900 \mathrm{~cm}^{-1}$ ) are the bleaching of the band-gap transition 73 and the formation of two mid-gap absorptions. 67 The high energy transient absorption at $10,900 \mathrm{~cm}^{-1}$ has a halfwidth of $1,600 \mathrm{~cm}^{-1}$, and the lower energy peak at $3,500 \mathrm{~cm}^{-1}$ has a halfwidth of $2,400 \mathrm{~cm}^{-1}$.

The two electronic absorptions are not believed to be associated with the same species because they have different dependences on the excitation wavelength. The excitation spectrum of the low energy peak increases sharply from just below $9,000 \mathrm{~cm}^{-1}$ to $12,000 \mathrm{~cm}^{-1}$, looking very much like the room temperature photoconductive response. 74 For excitation energies above $12,000 \mathrm{~cm}^{-1}$ the intensity of the photoinduced absorption at $3,500 \mathrm{~cm}^{-1}$ drops off, leveling off after $16,000 \mathrm{~cm}^{-1} .75$ The $10,900 \mathrm{~cm}^{-1}$ band is bleached at low excitation energies, begins to grow at $12,000 \mathrm{~cm}^{-1}$ and levels off around $24,000 \mathrm{~cm}^{-1}$. The low energy peak is believed to be due to the same species as the photoinduced IR active modes. Both absorptions have the same dependence on temperature, excitation chopping frequency, 68 and power. ${ }^{70}$

The rise time for photoproduction of the high energy peak has not been resolved, but is less than $150 \mathrm{fs} .73$ The time-resolved decay of the high energy absorption peak recorded at $11,700 \mathrm{~cm}^{-1}$ fits the form

$$
A(t)=\operatorname{erf} \sqrt{\tau_{\phi}}
$$

for the first 200 ps. The error function, 


$$
\operatorname{erf}(z)=2 / \sqrt{\pi} \int_{0}^{x} e^{-t} d t
$$

can be approximated as $t^{1 / 2}$ from 0.5 to $100 \mathrm{ps}$. The characteristic time, $t_{d}$, has a strong temperature dependence: $t_{d}=0.1 \mathrm{ps}$ at $20 \mathrm{~K}, \mathrm{t}_{d}=1.0 \mathrm{ps}$ at $300 \mathrm{~K}$. At lower probe energies, $10,000 \mathrm{~cm}^{-1}$ and $6,450 \mathrm{~cm}^{-1}$, the decay is faster, with an increasing fraction of the signal decaying at an exponential rate. At $6,450 \mathrm{~cm}^{-1}$ and $300 \mathrm{~K}$,

$$
\mathrm{A}(\mathrm{t})=0.74 \mathrm{e}^{-\mathrm{t} / 0.16 \mathrm{ps}}+0.26 \mathrm{erf} \sqrt{\tau_{\%}}
$$

The initial rise time for the low energy transient absorption peak is also unresolved. ${ }^{76}$ The signal decays almost completely within 15 ps at $300 \mathrm{~K}$. At $25 \mathrm{~K}$, the initial decay is similar, but followed by a slow second rise and decay centered at 40 ps. This second peak is much smaller for samples prepared by the Baker synthesis, which have fewer neutral defects.

\section{G.3 Electronic Properties of Photoexcited Polyacetylene}

As mentioned above, excitation above the band gap in room temperature transpolyacetylene results in a photoconductive response. The excitation spectrum for this response from $9,000 \mathrm{~cm}^{-1}$ to $12,000 \mathrm{~cm}^{-1}$ is the same as that for photoinduced absorptions at $3,500 \mathrm{~cm}^{-1}$ and in the mid-IR. The temperature dependence for photoconductivity is the reverse of that for photoinduced absorptions. The photoconductive response is strong at high temperatures and the photoinduced absorption is strong at low temperatures. There is also a low energy peak in the photoconductivity response curve near $9,000 \mathrm{~cm}^{-1} .77$ This peak does not have the same temperature dependence as the rest of the response curve. It is a minor feature at room temperature, but dominates the response curve at low temperatures.

There is no increase in conductivity in cis-polyacetylene on photoexcitation, but two strong, broad luminescence bands are observed at $14,900 \mathrm{~cm}^{-1}$ and $11,300 \mathrm{~cm}^{-1} .34$ The phenomena of photoconductivity and photoluminescence go hand in hand for normal semiconductor materials. They were believed to be mutually exclusive in the different polyacetylene isomers ${ }^{39}$ until recent reports of luminescence from the trans isomer at $18,900 \mathrm{~cm}^{-1} 78$ and $10,900 \mathrm{~cm}^{-1} .79$

\section{G.4 Electron Paramagnetic Resonance in Photoexcited Polyacetylene \\ No change has been observed in the intensity of the EPR signal during} photoexcitation above the band gap. .0 The sensitivity of the experiment was limited by the 
strong (Curie) dependence of the EPR signal intensity on temperature and the large dark signal. An excitation power known to saturate the photoinduced IR signal, and a chopping frequency observed to allow for complete decay of the photoinduced IR signal were used. The sample had a dark spin density of $10^{19} \mathrm{~cm}^{-3}$, compared to the photoinduced IR active defect density of $10^{17}-10^{18} \mathrm{~cm}^{-3}$. Within the sensitivity of the experiment it is possible to say that no more than $10^{16}$ new spins $\mathrm{cm}^{-3}$ were generated at $10 \mathrm{~K}$ and no more than $10^{15}$ $\mathrm{cm}^{-3}$ at room temperature.

\section{H.1 Prototype molecules}

To gain a better understanding of the origin and effect of bond altemation, or lack thereof, polyacetylene and doped polyacetylene it can be compared to well characterized prototype molecules. Polyacetylene when viewed as an infinite linear polyene chain can be compared to two types of prototype molecules. Infinite polyenes like cyclo-polyenes have no ends or end effects. Cyclooctatetraene and benzene are examples of cyclo-polyenes which do and do not exhibit bond altemation, respectively. Altematively, the properties of polyacetylene may be predicted from the limit of trends observed in a series of linear polyenes, increasing in chain length.

Benzene (Figure $1-3 \mathrm{~A}$ ) is planar, and has six $\pi$ electrons in three filled bonding orbitals, leaving three anti-bonding $\pi^{*}$ orbitals empty. All the bond angles are $120^{\circ}$ and all $\mathrm{C}$ - $\mathrm{C}$ bond lengths are equal, $\mathrm{r}=1.397 \AA$. For the $\mathrm{C}$ - $\mathrm{C}$ bonds to be equal the three $\pi$ bonding orbitals must be completely delocalized among all six bonds. If cyclooctaterraene were like benzene, it would be planar with eight $\pi$ electrons in three filled bonding orbitals and two half-filled non-bonding orbitals. The bond angles would be $135^{\circ}$, putting strain on the $\mathrm{sp}^{2}$ hybridized orbitals which prefer bond angles of $120^{\circ}$. Cyclooctatetraene is not planar but rather it found in a boat or tub form (Figure I-3B). This configuration has two distinct types of C-C bonds: those for which both adjacent bonds lie in the same plane, and those for which they do not. $\Pi$ bonding can only occur along bonds for which the adjacent bonds are in the same plane. Therefore the eight $\pi$ electrons form four $\pi$ bonds where they can, leading to bond altemation. It cannot be assumed that angle strain is the motivating force behind this distortion, since both the anion and the dianion (Figure $\mathrm{I}-3 \mathrm{C}$ ) of cyclooctaterraene are planar and do not exhibit bond alternation.

Cis-polyacetylene can be compared to cyclooctatetraene. In each there are two types of bonds in geometrically nonequivalent positions; therefore, bond alternation is expected. All the bonds in benzene, as in trans-polyacetylene, are in equivalent positions. Both have two strictly equivalent resonance forms and, in accordance with resonance 


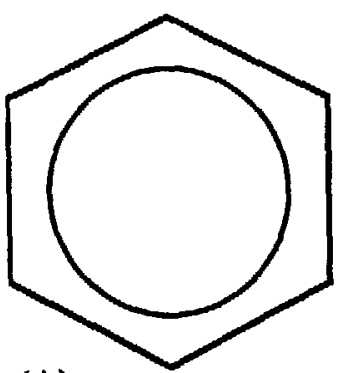

(A)

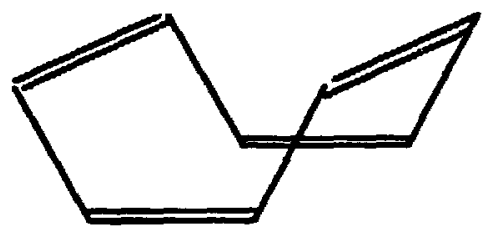

(B)
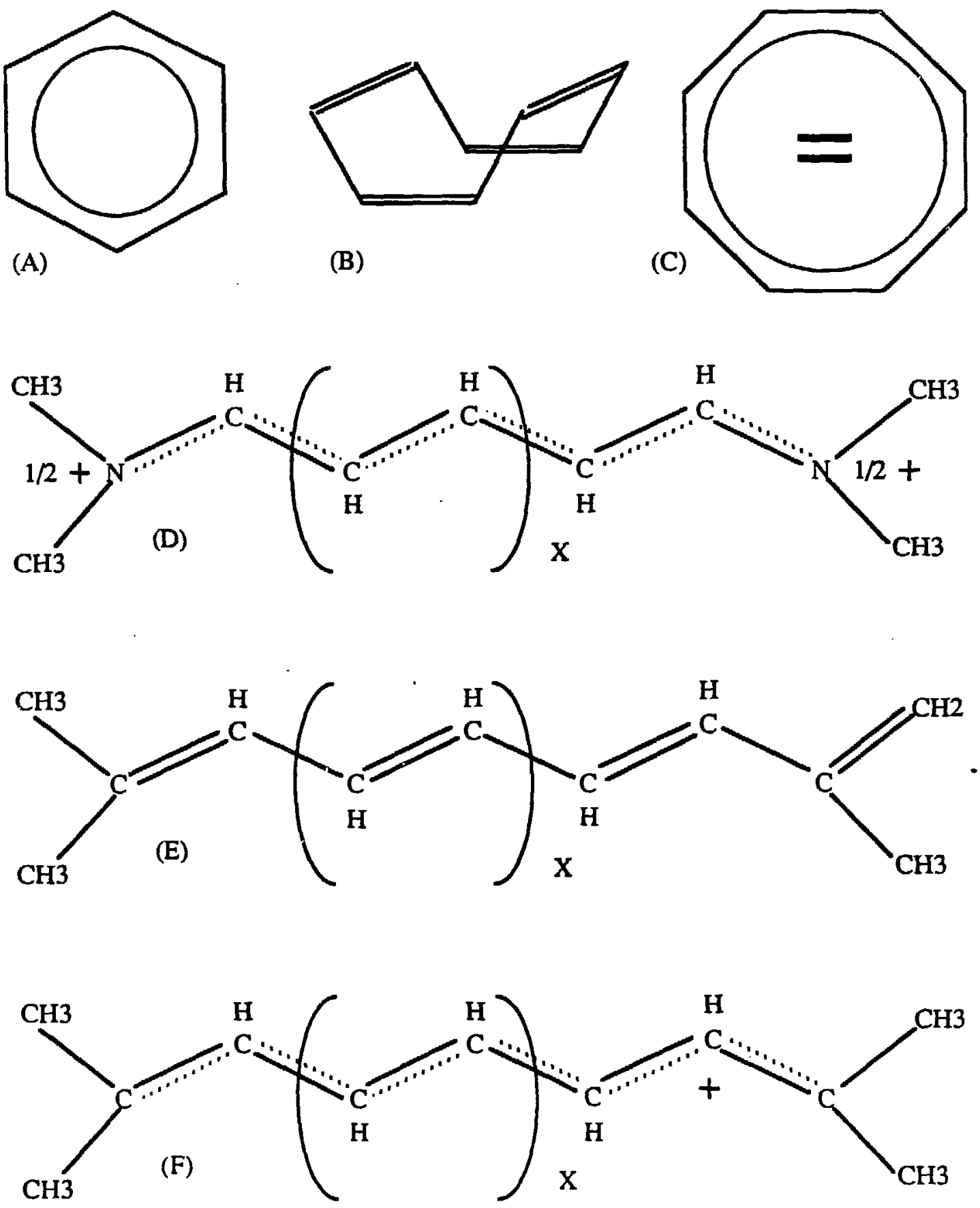

Figure I.3 Prototype Molecules

A) Benzene B) Cyclooctraterraene C) Dianionic form of cyclooctratetraene D) Cyanine dyes E) A series of linear polyenes F) The cationic forms of the same series of linear polyenes 
theory, are expected to adopt an intermediate form. Benzene behaves as expected; however, trans-polyacetylene does not.

It is necessary to look to the trends in linear polyenes to explain the bond alternation in trans-polyacetylene. Symmetric cyanine dyes (Figure I-3D) appear similar to trans. Both have two equivalent resonance forms. Unlike trans, cyanine dyes do not exhibit bond alternation. One distinguishing feature of cyanine dyes is that their wavelength for maximum absorption increases linearly with increasing chain length.

Sorensen ${ }^{81}$ prepared a series of linear polyenes (Figure I-3E). The absorption maxima for this series, shown in Figure I-4, are not linearly dependent on chain length. The change in the wavelength of the absorption maxima decreases as the chain length increases. This trend suggests a finite limit at $-450 \mathrm{~nm}$, where the absorption maxima stop changing with increasing chain length. The absorption maxima series of all trans- $\alpha-\omega$-dimethyl-polyenes approach a finite limit of $555 \mathrm{~nm}^{82}$ The absorption maximum observed in trans-polyacetylene is $653 \mathrm{~nm} .{ }^{39}$

Sorensen also examined the carbonium ions of this linear polyene series. The electronic transition energies for these carbonium ions were much lower than for the corresponding polyenes and were linearly related to the chain length, as were those of the cyanine dyes. A single but very broad NMR peak for hydrogens on the backbone of the carbonium ions suggest that all the $\mathrm{C}$-C bonds are nearly equivalent. The NMR spectra of the polyenes indicated a bond-alternated structure.

\section{H.2 Conjugation and Delocalization in Prototype Molecules}

Benzene has two equivalent resonance structures and, in accordance with resonance theory; adopts an intermediate form in which all $\mathrm{C}$ - $\mathrm{C}$ bonds are equal. The $\pi$ system in benzene can be modeled as a one-dimensional metal whose electrons are constrained to move on the circumference of a circle. With this model, the $\mathrm{C}-\mathrm{C}$ bond distance calculated from the electronic energy levels is $1.37 \AA, 83$ in reasonable agreement with the value, $r=$ $1.39 \AA$, obtained from rotational Raman studies. 84 Resonance theory also holds for the mono- and di-anionic forms of cyclooctatetraene, but not for cyclooctatetraene itself. The two half-filled non-bonding $\pi$ orbitals in cyclooctatetraene give it an electronically excited state nearly degenerate with the ground state, the difference being due to electron-electron repulsion. This state can mix with the ground state causing a pseudo Jahn-Teller distortion which lowers the energy of the ground state and raises the energy of the excited state. 85

Like benzene, symmetric cyanine dyes have two equivalent resonance structures and adopt an intermediate, non-bond altemating structure. These $\pi$ systems can also be modeled as one-dimensional metals with the electrons constrained to move along the chain. 


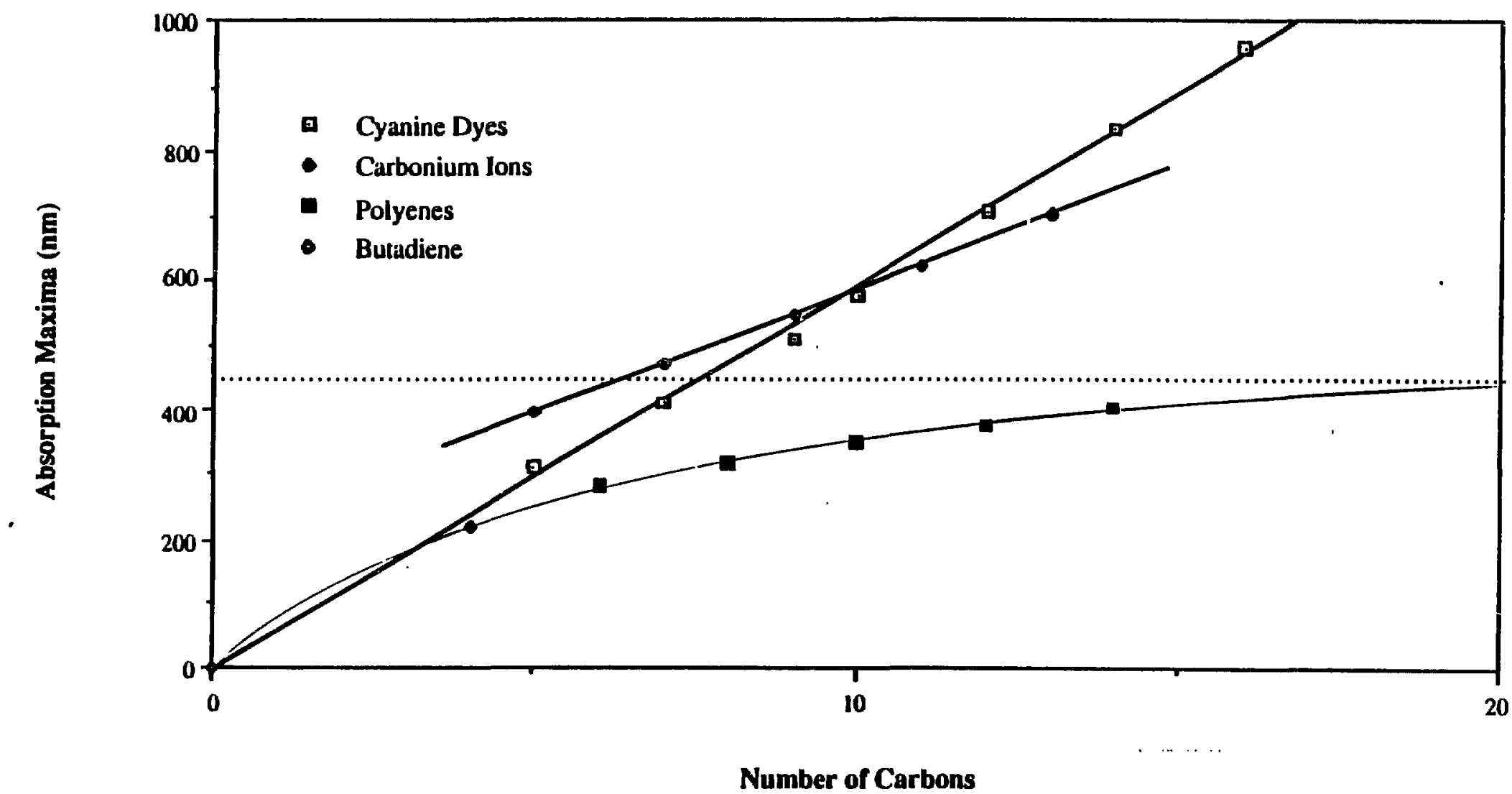

Figure I-4 Absorption Maxima as a Function of Chain Length

for cyanine dyes ${ }^{101}$ and a polyene series and the corresponding carbonions ${ }^{81}$ 
The electronic transition energies predicted with this model are in good agreement with those experimentally observed. The central portions of inear polyenes which are large enough for the end effects to be ignored do not behave like cyanine dyes although they have two equivalent resonance structures. In fact, as the polyenes get larger and the assumption that end effects can be ignored becomes more reasonable the deviation from metallic behavior increases.

Degenerate orbitals, such as the non-bonding orbitals in cyclooctatetraene, do not exist in linear systems; thus, the pseudo Jahn-Teller effect is responsible for bond alternation in linear polyenes. The energy of each orbital in a linear polyene is unique, but as the chain length increases the number of orbitals increases and the spacing between orbitals decreases. This results in an increasing number of excited states closer and closer to the ground state, as the molecular size increases. Bond alternation occurs when the energy gain from mixing electronic states provides a driving force strong enough to overcome the nuclear repulsive forces between adjacent carbon atoms. Thus, in longer molecules the mixing is more effective and a larger pseudo Jahn-Teller distortion is observed. The excited electronic states in cyanine dyes longer than 50 carbons are close enough that bond alternation is predicted .55 We are unaware of any work on dyes longer than 16 carbons, possibly because the absorptions of these dyes are at such low energy.

Solid-state physicists and most polyacetylene researchers refer to the pseudo JahnTeller effect as the Peierls effect. The carbonium ions made from Sorensen's polyenes are not subject to a Peierls distortion. The removal of an electron reduces the amount of energy which could be gained by mixing because the mixing lowers the energy of an unoccupied orbital. (Similarly, the addition of an electron reduces the amount of energy gained from mixing because the energy level of an occupied orbital is raised.) In these cases, the force created by the energy gain from mixing is too small to overcome the nuclear repulsive forces. In small molecules, bond alternation is lost throughout the chain. Larger molecules can contain both regions with bond alternation and charged, non-bond-alternated defects. The width of these defects is specifically determined by the balance between the relative strengths of the competing effects.

The $\mathrm{C}-\mathrm{H}$ units in bond-altemated polyenes are grouped in pairs by double bonds. Between regions where the double bonds are oriented differently there must be a segment containing an odd number of carbon atoms, no charge, and an odd number of electrons. The odd number of electrons means there must be one partially occupied orbital. This limits the amount of energy that can be gained by mixing because it costs energy to raise or lower a half-filled orbital. These misfit regions are expected to have similar structures to the non-bond-alternating, charged defects discussed above 


\section{I.1 Relationship Between Spin and Charge in Bond-Alternation Defects}

The non-bond-alternating defects discussed above have either a charge or an unpaired spin; this is a reversal of the normal relationship between spin and charge. The relationship between spin and charge in bond-altemating regions is more common; if the chain has an odd charge it has an unpaired spin, and if the chain is neutral it has no unpaired spin. The ability to support non-bond-alternating defects makes long bondalternating polyenes (e.g. trans-polyacetylene) behave as two-phase systems. The defect can be thought of as an excursion from the normal spin/charge relationship phase of the bond-altemated region into the reversed spin/charge relationship phase and back. This excursion is viewed as a solitary wave. The energy and size of this excursion are fixed quantities, giving the wave particle-like properties. Such a solitary wave is known as a soliton.

\section{I.2 History of Solitons}

The first recorded observation of a solitary wave was made by John Scott Russell in 1834.86 A lurching barge created a mound-like disturbance in the water of a narrow shipping channel. Russell observed the disturbance as it propagated along the channel for several miles without any change in form, size or velocity. Korteweg and deVries worked out the mathematical description of this phenomenon in 1895. Most of the mathematics for solitons in other systems were worked out in the mid-1960's, after the importance of solitons in non-linear effects was recognized.

The following general features of solitons are worthy of note. Their energy is constant. They are stable and do not dissipate. Solitons can propagate, without any change in size or form, at a velocity which is proportional to their amplitude. Solitons have non-linear interactions with other solitons yet emerge from these interactions in their original state, except for a small change in their time-distance phase.

In the general case, solitons in one-dimensional, two-phase systems are solutions to the Sin-Gordon equation. In a simple Huckel treatment of trans-polyacetylene, solitons are solutions to the Su-Schrieffer-Heeger Hamiltonian.87,88 Another solution to this Hamiltonian, which may play a role in the properties of polyacetyiene, is a polaron. Polarons can be visualized as two partially overlapping solitons, one neutral and one charged. This results in a wider non-bond-alternating defect, with a bimodal charge distribution. ${ }^{89}$ The direction of bond alternation on either side of a polaron is the same. Polarons do not involve any change in the spin/charge relationship. Neutral polarons in linear polyenes, with even numbers of equivalent carbon-carbon bonds, should be unstable 
because of the Peierls or pseudo Jahn-Teller effect. Considerably less theoretical work has been done on polarons than on solitons.

\subsection{Mobility of Bond-Alternation Defects}

When a soliton moves along the chain the direction of bond alternation is converted in its wake. The two bond-alternated forms of trans-polyacetylene, double bonds to the left or right, are mirror images of each other and energerically degenerate. Since there is no energy difference between the initial and final structures, the only barrier to soliton movement is a small activation energy associated with the transitional form of the soliton not centered on a carton atom. The activation energy calculated using the Su-SchriefferHeeger Hamiltonian is $16 \mathrm{~cm}^{-1} .90$ This low barrier is consistent with the dynamic nuclear polarization experimental result that defects are mobile at temperatures down to $30 \mathrm{~K}^{51}$

The two isomers of cis-polyacetylene are not energetically degenerate. An odd carbon non-bond-alternating defect in a stable position on a cis segment (at the end) would convert the part of the chain it passed over to a higher energy form as it moved. Defects in cis-polyacetylene are expected to be immobile because of the net change in energy associated with their movement.

There is no net energy change associated with the displacement of a polaren because the bond altemation is the same on both sides of it. An activation energy and mobility similar to that calculated for a soliton can be assumed for a polaron.

The effective mass of a moving soliton is calculated from the expression $\mathbf{M}_{\mathbf{s}}=$ $\mathrm{M}_{\mathrm{CH}}\left(\mathrm{u}_{\mathrm{O}}\right)^{2 / a l}$, where $\mathrm{M}_{\mathrm{CH}}$ is the mass of a $\mathrm{C}-\mathrm{H}$ unit; $u_{0}$ is the symmetry breaking parameter $-0.03 \AA ; a$ is the average $C-C$ bond length; and $l$ is the soliton halfwidth, $\sim 7$ bond lengths. ${ }^{87,88}$ This gives the surprising result that, despite the change in nuclear positions associated with the movement of a soliton, the calculated effective mass of a soliton is about the same as the mass of an electron. The effective mass derived from dynamic nuclear polarization experiments ${ }^{50}$ agrees with this finding.

\section{I.t Length of Bond-Alternation Defects}

The half-width of a soliton calculated using the Su-Schrieffer-Heeger Hamiltonian is $l=7$ carbons. ${ }^{90}$ Calculations performed with Pariser-Parr-Pople ${ }^{91}$ and modified neglect of differential overlap corrections ${ }^{89}$ for electron-electron repulsion predict narrower defects: $l=5$ carbons for neutral and cationic defects, $l=3$ carbons for anionic defects. Polarons are expected to have a larger half-width: $l=7$ carbons for the anion, $l=9$ carbons for the cation. 89 


\section{I.5 Electronic Structure of Bond-Alternation Defects}

The energy of a soliton, calculated using the Su-Schrieffer-Heeger Hamiltonian, is $E_{s}=2 \Delta \pi, 92$ where $\Delta$ is the energy required to add an electron from the Fermi level, or remove an electron to the Fermi level, or half the band-gap energy. At this level of theory, there is an electronic state associated with any of the three types of solitons $5,000 \mathrm{~cm}^{-1}$ above the valence band, for a band gap of $15,700 \mathrm{~cm}^{-1}$ (Figure I-5A). Corrections should be made to put the more oxidized species at higher chemical potential. The extra electron in an anionic defect is the second in the highest occupied orbital. The energy of an electron in a filled orbital is higher than that of a single electron in the same orbital because of electronelectron repulsion. The highest occupied orbital in a cationic defect is a lower energy orbital than the highest occupied orbital in a neutral or anionic defect. Furthermore, the electrons in the more oxidized species are less tightly bound because the electron to positive charge ratio is higher.

The defects themselves have several unoccupied orbitals which are responsible for a series of low-lying excited electronic states. Transitions to these states should be much less intense than those to the massive density of states available in the valence and conduction bands. Absorptions between $4,000 \mathrm{~cm}^{-1}$ and 7,000 $\mathrm{cm}^{-1}$ have been observed for both $p$ and $n$-doped polyacetylene. If the concentration of neutral defects in undoped polyacetylene is as high as EPR experiments suggest, the electronic absorptions of the neutral defects should also be observable. However, only a weak photoacoustic absorption at $5950 \mathrm{~cm}^{-1}$, assigned to a molecular vibration, has been observed.

When polarons are viewed as two overlapping solitons, the nearly degenerate electronic states associated with the neutral and charged solitons mix strongly. The final picture (Figure I-5B) has two states, one near the valence band and one near the conduction band. ${ }^{93}$ Strong near-IR absorptions would be expected between these states and the high density of states in the valence and conduction bands. At low defect concentrations the absorption between the two states of the defect should be weak.

Viewed as a single entity a polaron is associated with a single mid-gap electronic state. Using a band gap of $11,300 \mathrm{~cm}^{-1}$ the electronic states calculated for for positively and negatively charged polarons are $2,400 \mathrm{~cm}^{-1}$ above the valence band and below the conduction band, respectively $89\left(3,200 \mathrm{~cm}^{-1}\right.$ for $\left.E_{g}=15,700 \mathrm{~cm}^{-1}\right)$. Two near-IR absorptions, at 3,200 and $12,500 \mathrm{~cm}^{-1}$, should be observed for the transitions between these states and the valence and conduction bands.

Like-charged polarons on the same chain are believed to be unstable with respect to two charged and two neutral solitons. ${ }^{94}$ Thus, at high defect concentrations, soliton formation is favored over polaron formation. At low concentrations the energy of a 
(a)
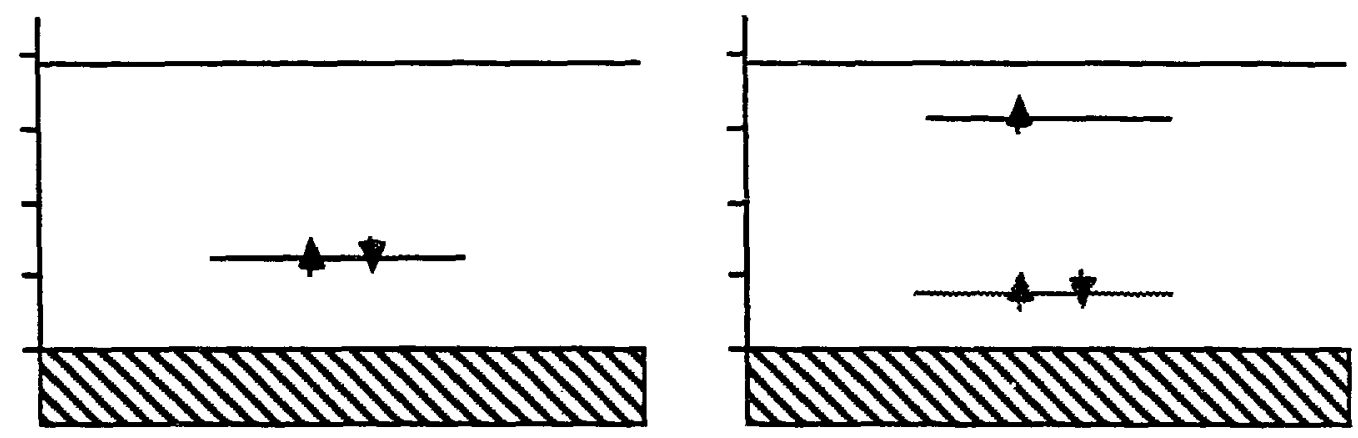

(b)

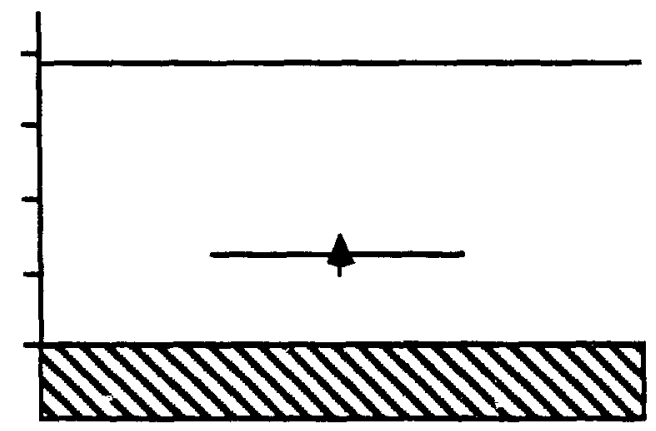

(c)
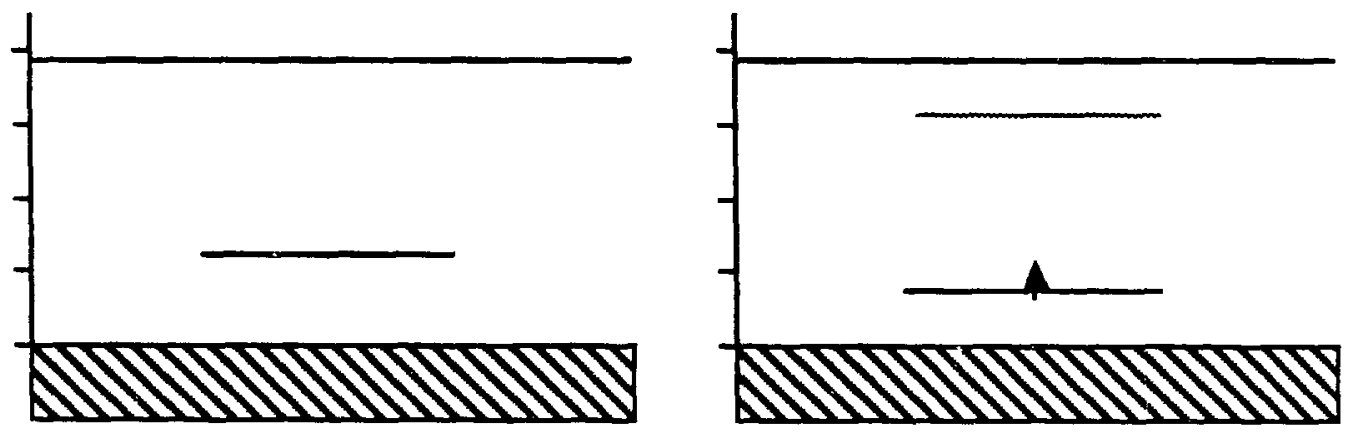

(A)

(B)

Figure I.5 Predicted Energy Levels for Bond-Alternation Defects

A) Solitons, located $5,000 \mathrm{~cm}^{-1}$ above the band-gap a) anionic b) neutral c) cationic B) Polarons a) anionic, with a half-filled state $2,400 \mathrm{~cm}^{-1}$ below the conduction band c) cationic, with a half-filled state $2,400 \mathrm{~cm}^{-1}$ above the conduction band. The filled and empty states shown in gray are artifacts of the two overlapping solitons picture of polarons. 
polaron is predicted to be lower than the energy of two solitons. Thus, at low dopani levels, polaron formation is favored over the formation of two solitons in circumstances where a change in the direction of bond alternation is not otherwise imposed. In spite of this prediction, the electronic absorptions observed in lightly doped polyacetylene are commonly attributed to charged solitons. The frequency shift from 7,000 to $4,000 \mathrm{~cm}^{-1}$ with increasing dopant concentrations may be due to a change in the relative populations of polarons and solitons.

\section{I.6 Vibronic Enhancement}

The mid-IR absorptions of dopant- and photoinduced defects are unusually intense. It is widely accepted that the charge on the defect is responsible for the large oscillator strength. The molecular vibrations shift the excess charge, causing an unusually large dipole derivative. Uncharged defects with the same structures are expected to have much weaker IR absorptions. The effect of the excess electronic charge on the intensity of the vibrational absorptions is known as vibronic enhancement. The results of recent calculations ${ }^{57}$ of the frequencies and relative intensities expected for charged, non-bondaltemated defects in trans-polyacetylene are given in Table I-3. The predicted frequencies for the C-C stretching "sliding" modes of solitons and polarons are identical. Additional absorptions with similar oscillator strengths are expected for polarons.

Photo- and dopant-induced defects have strong absorptions near $1390 \mathrm{~cm}^{-1}$ and $1290 \mathrm{~cm}^{-1}$ commonly attributed to vibrations of charged solitons. Very recently, additional weak absorptions at $1438 \mathrm{~cm}^{-1}\left(1340 \mathrm{~cm}^{-1}\right.$ in $\left.(C D)_{x}\right)$ and $1034 \mathrm{~cm}^{-1}\left(858 \mathrm{~cm}^{-1}\right.$ in $\left.(C D)_{x}\right)$ have been observed by high sensitivity FT-IR spectroscopy (signal averaging for 15-45 hrs.) $)^{69}$ of above-gap photoinduced defects. Because of their lack of intensity these absorptions are attributed to phonon coupling to the uniform translational mode of a charged soliton. The frequencies of these absorptions match those predicted for the additional IR active modes of polarons. The possibility that the oscillator strengths for these modes may have been overestimated or that above-gap photoexcitation produces a mixture of solitons and polarons should not be overlooked. The relative intensities of these new absorptions and those previously observed should be examined as a function of excitation wavelength, near the absorption onset and above the band gap, to determine if there are two types of photoinduced defects.

\subsection{The Pinning Mode}

The lowest energy $\mathbb{R}$ absorption of the dopant-induced defects in transpolyacetylene is at $832 \mathrm{~cm}^{-1}\left(670 \mathrm{~cm}^{-1}\right.$ in $\left.(C D)_{x}\right)$. This was originally attributed to a 
Raman mode of trans made IR allowed by the symmetry-breaking dopant ion. ${ }^{56}$ It was suggested that the large shift from the Raman absorption frequencies at $1072 \mathrm{~cm}^{-1},(765$ $\mathrm{cm}^{-1}$ for $(C D)_{\mathbf{x}}$ ) to lower frequency is due to the pinning potential of (Coulombic attraction to) the dopant ion. 95

This band is unusually wide for a molecular vibration, $\sim 400 \mathrm{~cm}^{-1}$. The absorption is now believed to be due to the vibration of the defect back and forth along the polymer chain about the dopant ion. The restoring force for this vibration is the Coulombic attraction between the charged defect and the counterion. ${ }^{58}$ It was suspected that in the absence of the dopant ion this vibration would have zero frequency; however, photoinduced defects have similar broad, low-energy absorptions at $536 \mathrm{~cm}^{-1}\left(510 \mathrm{~cm}^{-1}\right.$ in $\left.(\mathrm{CD})_{\mathbf{x}}\right)$.

A pinning mechanism, involving the Coulombic attraction between oppositely charged defects, has been proposed for photoinduced defects. With values for the effective mass of the defect varying from $1^{39}$ to $6^{87}$ times the mass of an electron, it is not surprising that separate calculations put the defects on nearest neighbor chains, $4 \AA$ away, 58 and next nearest neighbor chains, $7.3 \AA$ away.96 These calculations do not address the difference in the isotope shifts observed in dopant-induced and photoinduced defects, 1.25 and 1.05 , respectively. One calculation predicts a difference in the effective masses of defects on $(\mathrm{CH})_{x}$ and $(\mathrm{CD})_{x}, 2.7 \mathrm{~m}_{e}$ vs. $2.9 \mathrm{~m}_{e}$, respectively. 95

\section{I.8 Formation of Bond-Alternation Defects}

Thermal isomerization of polyacetylene or exposure to $\mathrm{O}_{2}$ results in the development of an EPR signal at $\mathbf{g}=2$. This signal is indicative of organic free radicals such as neutral solitons and charged polarons. No accompanying IR signal, which would indicate a charged defect, is developed. Thus thermal isomerization and exposure to $\mathrm{O}_{2}$ are believed to induce the formation of neutral solitons.

When polyacetylene reacts with either an electron donor or acceptor a non-bondalternating defect should be formed to accommodate the charge. The formation of these defects causes an increase in the sample's conductivity, suggesting that these defects are the charge carriers. Intense IR bands also develop at 1400,1290 and $900 \mathrm{~cm}^{-1}$, indicating a charged defect. The band-gap transition is suppressed and a new electronic transition develops near $5,000 \mathrm{~cm}^{-1}$, suggesting the formation of mid-gap defects.

Because the direction of bond alternation is changed by solitons, they must be formed in pairs. The formation of a charged soliton accompanied by the formation of a neutral soliton, seems more probable than the simultaneous formation of two charged solitons. Thus an increase in the EPR signal is expected on light doping of polyacetylene if 
soliton pairs are produced. If neutral solitons are the preferred sites for oxidation or reduction, a decrease in the EPR signal is expected. No change is observed in the EPR signal for $y<0.001$. At higher concentrations the EPR signal disappears. This is consistent with the formation of polarons at low dopant concentrations, followed by the formation of charged solitons, preferentially from neutral solitons.

\section{I.9 Photoproduction of Bond-Alternation Defects}

The energy of a single charged soliton is predicted to be below the center of the band gap. Thus the energy of a pair of charged solitons should be less than the energy of an electron-hole pair. Real-time integration of the equations of motion for transpolyacetylene using the Su-Schrieffer-Heeger Hamiltonian with electron-phonon coupling ${ }^{88}$ predict that electron-hole pairs relax to charged soliton pairs in $\sim 10^{-13}$ seconds. This treament predicts polaron formation for the injection of a single charge. Thus, if the electron and hole created by photoexcitation are not confined to the same chain, the formation of two charged polarons is expected. If the excitation were confined to a single chain the formation of two charged solitons would be expected. It has been suggested that even weak electron-electron interactions can break the charge-conjugation symmetry which requires that these solitons be charged.97 Thus it may be possible to produce charged solitons, neutral solitons, or charged polarons with photoexcitations above the band gap. It has also been suggested that the $10,900 \mathrm{~cm}^{-1}$ shoulder is due to electronic absorption of neutral defects and that these defects mediate the photoproduction of defects or are photoconverted to charged defects. ${ }^{75}$

Low band-edge luminescence yields indicate that photogenerated electron-hole pairs follow non-radiative decay pathways such as the formation of defects. Existing experimental evidence suggests a great deal about what mechanisms are and are not possible for the formation of photoinduced defects. The development of strong IR absorptions suggests that charged defects are created. Vibronic enhancement would account for the intensity of the $\mathrm{IR}$ absorptions. The similarities in the absoprtion spectra of photoinduced and dopant-induced defects and the constant integrated absorption intensity observed during photoexcitation of lightly doped polyacetylene suggest that similar defects are produced by doping and photolysis. Bleaching of the interband transition during above gap excitation suggests that bond-altemating regions are lost during defect formation. The temperature dependence for photoinduced absorption and photoconductivity at these excitation energies are opposite. Bleaching near $10,900 \mathrm{~cm}^{-1}$ suggests a different mechanism for the production of photoinduced defects with sub-gap excitation. With this production mechanism the photoconductive response is not traded for photoinduced 
absorption intensity. The time evolution of the intensity of the low energy electronic absorption indicates two mechanisms for the same (above gap) excitation energy. The fact that the EPR signal is constant to within one tenth of a percent, requires all mechanisms to maintain a balance of charged polarons and neutral solitons.

\section{I.10 Relationship Between Bond-Alternation Defects and Conductivity \\ Epstein et al. 45 have compared the d.c. and a.c. conductivity data for undoped} polyacetylerie to four model systems: thermal activation of extended states, thermal activation of hopping in band tails, polaron hopping in an ordered lattice, and hopping in a manifold of states at the Fermi level. The d.c. conductivity data fit the model for hopping in a manifold of states at the Fermi level. The observed temperature dependence of the a.c. conductivity is much stronger than that predicted with any model. At any given temperature, the frequency dependence fits all models except that for thermal activation of extended states. The model most consistent with the data is hopping in a manifold of states at the Fermi level. This suggests isolated defects, such as solitons, with electronic states near the center of the band gap which are capable of accommodating a charge.

There is a gradual transition from this type of hopping behavior to a more metallic behavior as the dopant level is increased. 98 Assuming non-bond-alternating defects approximately 10 carbons long, the localized states would overlap at dopant concentrations $y>0.1$. This would constitute a Mott insulator-metal transition among localized carriers. ${ }^{99}$ At intermediate dopant concentrations, $y=0.05-0.10$, some states overlap and some do not, causing mixed behavior. True metallic behavior, where the conductivity increases when the temperature decreases, has not been reported for doped polyacetylene. Chain imperfections and finite chain lengths may be the limiting factors. It is possible that a metallic temperature dependence will be observed in doped polyacetylene prepared by the Narrmann technique or some synthesis yet unknown.

\section{$\mathrm{J}$ Science and technology}

While quite a few polymers and crystalline organic solids are known to conduct electricity, polyacetylene has become the primary target for research because of its simplicity. Early polyacetylene research was further fueled by hopes of, and patents for, lightweight batteries, inexpensive solar paneis, stabilized electrodes, electromagnetic and electrostatic shielding, and semiconductor devices. ${ }^{39}$ High conductivity and mid-gap defects with three stable oxidation states make polyacetylene a prime choice for electrodes in lightweight batteries. The near perfect match between the solar spectrum and the 
absorption spectrum of trans-polyacetylene meet one important requirement for efficient photovoltaic cells.

Advances in polyacetylene synthesis have brought some of these hopes closer to reality. Stretch alignment can be used to increase the conductivity (in one direction), but does little to ease the difficulties in processing. Baker's synthesis deals with the processing problem, but to no technological advantage since the conductivity is effectively lost. However, the Baker synthesis does offer an opportunity to study the effect of the morphology and the number of neutral spins on the properties of polyacetylene by drastically altering these two factors. The precursor routes offer the greatest ease of processing, but again the electrical properties suffer, and until this is overcome the advantages for applications are lost. Again the different morphology offers the opportunity to study its effect on the polymer. The Narmann synthesis, on the other hand, has proven a technological victory. 22 When doped, samples produced ui this manner have twice the conductivity of copper by weight. This development has made batteries with polyacetylene electrodes a reality.

Along with scientific and technological advances have come new ideas for applications. ${ }^{22}$ The color change which accompanies the thermal cis-trans isomerization of polyacetylene is used as an indicator for heat sensitive drugs which must be kept at low temperatures during shipping. The high conductivity and chemical stability of polyacetylene make it a good candidate for synthetic nerves.

Some of the problems associated with using polyacetylene in various applications may be overcome by abandoning it for soluble polymers, with similar backbones, which can also be doped to conductive states. Such polymers are being developed and studied; however, for basic research polyacetylene is still the best system to study. The fact that polyacetylene is nothing more than a carbon chain with alternating double and single bonds, the bare essentials for a conducting polymer, make it the "prototype conducting polymer". There are no side groups to complicate the electronic properties or vibrational, electronic, and magnetic spectra. The effects of certain physical properties on the behavior of the polymer can be studied because the different syntheses produce somewhat varied samples.

In this thesis, spectroscopic methods are used to study the response of polyacetylene to photolysis in the band gap at low temperatures. These studies reveal the location and some physical and chemical characteristics of the mid-gap defect states in undoped polyacetylene. In addition, attempts to prepare polyacetylene and polyacetylenelike polymers via solid-state polymerization, as well as various spin-off experiments related to the electronic structure of the acetylene monomer, are described and discussed in Chapter 
II. Experimental work on the formation and characterization of defect states in transpolyacetylene are presented in Chapter II. The thermal barrier to the chemical doping reaction was characterized by the temperature required for the reaction to take place at an observable rate, for several dopant molecules. These temperatures were compared to the temperatures required to dope samples during photolysis with above-band-gap light. It was anticipated that the production of photoinduced charged defects would facilitate the doping process. The $\mathbb{R}$ absorptions of photoinduced defects with much longer lifetimes than those previously reported were observed. The electronic structure of these defects was characterized by the photolysis frequencies which could produce and destroy them. These defects were produced at lower photolysis energies than the transient photoinduced defects previously reported. The intensity of their IR absorptions and the rate at which these absorptions decayed was measured as a function of temperature and sample isomer content. These experiments and their results are discussed in Chapter IV. 


\section{References}

1 G. B. Street, T. C. Clark, IBM J. Res. Develop. 25(1), 51 (1981)

2 A. J. Heeger, A. G. MacDiarmid, Synth. Metals 1, 101 (1979-1980)

3 Y.-W. Park, A. J. Heeger, M. A. Druy, A. G. MacDiarmid, J. Phys. Chem. 73(2), 946 (1980)

4 W. Reppe, O. Schichting, K. Klager, T. Toepel, Ann. 560, 104 (1948)

5 H. Haberkorn, H. Naarmann, K. Penzien, J. Schlag, P. Simak, Synth. Metals 551 (1982)

6 S. C. Lind, D. C. J. Bardwell, J. Am. Chem. Soc. 48, 1575 (1926)

7 Y. Tabata, B. Saito, H. Shibano, H. Sobue, K. Oshima, Macromolek Chem. 76, 89 (1964)

$8 \quad$ S. Shida, Z. Kuri, T. Furaaoya, J.C.P., 28(1), 131 (1958)

9 H. Shirakawa, T. Kobayashi, J. Phys. Coll. C3, 443 (1983)

10 E. Tsuchida,C -N. Shih, I. Shinohara, S. Kambara, J. Poly Sci. A2, 3347 (1961)

11 T. Ito, H. Shirakawa, S. J. Ikeda, Polym. Sci., Polym. Chem. Ed. 12, 11, (1974)

12 M. A. Druy, C.-H. Tansg, N. Brown, A. J. Heeger, A. G. MacDiarmid, J. Polym. Sci. Polym. Phys. Ed. 18,429 (1980)

13 K. Araya, A. Mukoh, T. Narahara, H. Shirakawa, Chem. Lett. 1141 (1984)

14 G. L. Baker, J. A. Shelburne III, F. S. Bates, J. Am. Chem. Soc. 108, 7377 (1986)

15 J. H. Edwards, W. J. Feast, Polymer 21, 595 (1980)

16 G. Leising, Polym. Comm. 25, 201 (1984)

17 G. Leising, Polym. Bull. 11, 401 (b) (1984)

18 M. E. Horton, D. D. C. Bradley, R. H. Friend, C. K. Chai, D. C. Boot, Mol. Cryst. Liq. Cryst. 117, 51 (1985)

19 K. Harper, P. G. James, Mol. Cryst. Liq. Cryst. 117, 55 (1985)

20 T. M. Swager, D. A. Dougherty, R. H. Grubbs, J. Am. Chem. Soc. 110, 2973 (1988)

21 N. Basescu, Z.-X. Liu, D. Moses, A. J. Heeger, H. Naarmann, N. Theophilou, Nature (london) 327(4), 403 (1987)

22 R. B. Kaner, A. G. MacDiarmid, Sci. Am. 258(2), 106 (1988) 
23 J. C. W. Chien, M. A. Schen, J. Polym, Sci., Polym. Chem. Ed., 23, 2447 (1985)

24 J. C. W. Chien, Polyacetylene Chemistry, Physics, and Material Science, (Academic Press: Orlando,1984) p.49

25 F. B. Schugerl, H. Kuzmany, J. Chem. Phys. 74(2), 131 (1981)

26 H. Shirakawa, S. Ikeda, Polymer J. 2(2), 231 (1971)

27 T. Akaishi, K. Miyasaka, K. Ishikawa, J. Polym. Sci., Polym. Phys. Ed. 18, 745 (1980)

28 C. K. Chiang, A. J. Heeger, A. G. MacDiarmid, Ber. Bunsenges. Phys. Chem. 83, 407 (1979)

29 J. C. W. Chien, J. D. Capistran, F. E. Karasz, L. C. Dickinson, M. A. Schen, J. Polym. Sci., Polym. Lett. Ed. 21, 93 (1983)

30 M. A. Schen, F. E. Karasz, J. C. W. Chien, Makromol. Chem., Rapid Commun. 5, 217 (1984)

31 M. A. Schen, F. E. Karasz, J. C. W. Chien, J. Polym. Sci., Polym. Chem. Ed. 21, 2787 (1983)

32 G. Perego, G. Luugli, U. Pedretti, Mol. Cryst. Liq. Cryst. 117, 59 (1985)

33 J. C. W. Chier, Polyacetylene Chemistry, Physics, and Material Science, (Academic Press: Orlando,1984) p.46

34 C. R. Fincher Jr., C.-E. Chen, A. J. Heeger, A. G. MacDiarmid, J. B. Hastings, Phys. Rev. Lett. 48(2), 100 (1982)

35 H. Takeuchi, Y. Furakawa, I. Harada, H. J. Shirakawa, Chem. Phys. 84(5), 282 (1986)

36 H. Takeuchi, T. Arakawa, Y. Furakawa, I. Harada, H. Shirakawa, J. Mol. Structure 158, 179 (1987)

37 L. S. Lichtman, E. A. Imhoff, A. Sarhang, D. B. J. Fitchen, Chem. Phys. 81(1), 168 (1984)

38 I. Harada, M. Tasumi, H. Shirakawa, S. Ikeda, Chem. Letts. 1411 (1978)

39 S. Etemad, A. J. Heeger, A.G. MacDiarmid, Ann. Rev. Phy. Chem., 33, 443 (1982)

40 J. Tanaka, M. Tanaka, H. Fujimoto, K. Kamiya, Mol. Cryst. Liq. Cryst. 117, 259 (1985)

41 H. Eckhardt, R. R. Chance, Phys. Chem. 79(11), 5698 (1983) 
42 S. Etemad, G. L. Baker, C. B. Roxlo, B. R. Weinberger, J. Orenstein, Mol. Cryst. Liq. Cryst. 117, 275 (1985)

43 N. Suzuki, M. Ozaki, S. Etemad, A. J. Heeger, A. G. Macdiarmid, Phys. Rev. Lett. 45, 1209 (1980)

44 H. Shirakawa, T. Ito, S. Ikeda, Makromol. Chem. 79, 1565 (1978)

45 A. J. Epstein, H. Rommelmann, M. Abkowitz, H. W. Gibson, Mol. Cryst. Liq. Cryst. 77, 81.(1981) A. J. Epstein, H. Rommelmann, M. A. Dury, A.J. Heeger, A. G. MacDiarmid, S. S. Commun. 38, 683 (1981)

46 P. Bernier, M. Rolland, C. Linaya, M. Disi, Polymer 21, 7 (1980)

47 J. C. W. Chien, F.E. Karasz, G.E. Wnek Nature (london) 285, 390 (1980)

48 B. R. Weinberger, E. Ehrenfreund, A. Pron, A, J. Heeger, A. G. Macdiarmid, J. Chem. phys. 72, 4749 (1980)

49 H. Thomann, G. L. Baker, J. Am. Chem. Soc. 109, 1569 (1987)

50 M. Nechtschein, F. Devreux, R. L. Green, T. C. Clark, G. B. Street, Phys. Rev. Lett. 44(5), 356 (1980)

51 W. G. Clark, K. Glover, G. Mozurkewich, Mol. Cryst. Liq. Cryst. 117, 447 (1985)

52 J. C. W. Chien, Polyacetylene Chemistry, Physics, and Material Science, (Academic Press: Orlando,1984)

53 J. F. Rabolt, T. C. Clark, G. B. Street, J. Chem Phys. 71(11), 4614 (1979)

54 C. R. Fincher Jr., M. Ozaki, M. Tanaka, D. Peebles, L. Lauchlan, A.J. Heeger, A. G. MacDiarmid, Phys. Rev. B20, 1589 (1979)

55 H. Fujimoto, J. Tanaka, M. Tanaka, Bull. Chem. Soc. Jpn. 56, 671 (1983)

56 J. F. Rabolt, T. C. Clark, G. B. Street, J. Chem Phys. 76(12), 5781 (1982)

57 E. J. Mele, J. C. Hicks Phys. Rev. B32(4), 2703 (1985)

$58 \quad$ R. J. Cohen, A. J. Glick, Phys. Rev. B36(50), 2907 (1987)

59 H. Eckhardt, L. W. Schacklette, J.S. Szobota, R. H. Baughman, Mol. Cryst. Liq. Cryst. 117, 401 (1985)

60 H. Shirakawa, T. Sasaki, S. Ikeda, Chem. Lett. 1113 (1978)

61 G. Mihaly, G. Vancso, S. Pekkev, A. Janossy, Synth. Metals 1, 357 (1979/1980)

62 N. F. Mott, E. A. Davis,Electronic Processes in Non-crystalline Mnterials, (Clarendon Press: Oxford, 1971) p.264 
63 S. Ikehata, J. Kaufer, T. Woerner, A. Pron, M. A. Dury, A. J. Heeger, A. G. MacDiarmid, Phys. Rev. Lett. 45(13), 1123 (1980)

64 J. M. Pochan, D. F. Pochan, H. Rommelmann, H. W. Gibson, Macromol. 14, $110(1981)$

65 J. C. W. Chien, Polyacetylene Chemistry, Physics, and Material Science, (Academic Press: Orlando,1984) p.178

66 S. Lefrant, E. Rzepka, P. Brenier, M. Rolland, M. Aldissi, Polymer 21, 1235 (1980)

67 J. Orenstein, G. L. Baker, Phys. Rev. B49(14), 1043 (1982)

68 Z. Vardeny, J. Orenstein, G. L. Baker, Phys. Rev. Lett. 50(25), 2032 (1983)

69 H. E. Schaffer, R. H. Friend, A. J. Heeger, Phys. Rev. B36(14), 7537 (1987)

70 G. B. Blanchet, C. R. Fincher, T. C. Chung, A. J. Heeger, Phys. Rev. Lett. 50(24), 1938 (1983)

71 E. Ehrenfreund, Z. Vardeny, O. Brafman, R. Weagley, A. J. Epstein, Phys. Rev. Lett. 57(16), 2081 (1986)

72 J. Orenstein, Z. Vardeny, G. L. Baker, G. Eagle, S. Etemad, Phys Rev. B30(2), 786 (1984)

73 C. V. Shank, R. Yen, R. L. Fork, J. Oreistein, G. L. Baker, Phys. Rev. Letts. 49, 1660 (1982)

74 G. B. Blanchet, C. R. Fincher, A. J. Heeger, Phys. Rev. Lett. 51(23), 2132 (1983)b

75 J. Orenstein, Chapter 36, T. Skotheim Ed.,The Handbook of Conducting Polymers;; (M. Dekker, New York 1986)

76 L. Rothberg, T. M. Jedju, S. Etemad, G. L. Baker, Phys. Rev. Lett. 57(25), 3229 (1986)

77 B. R. Weinberger, Phys. Rev. Lett., 50(21) 1693, (1983)

78 K. Iwahana, P. Knoll, H. Knzmany, Mol. Cryst. Liq. Cryst. 117, 255 (1985)

79 P. W. Carter, private communication

80 J. D. Flood, A. J. Heeger, Phys. Rev. B28(5), 2356 (1983)

81 T. S. Sorensen, J. Am. Chem. Soc. 87(22), 5076 (1965)

82 L. Salem, The Molecular Orbital Theory of Conjugated Systems, (W. A. Benjamin, Inc., New York,1966) p. 366

83 T. W. Simpson, J. Chem. Phys. 16(12), 1124 (1948) 
84 B. P. Stoicheff, Can. J. Phys. 32, 339 (1954)

85 L. Salem, The Molecular Orbital Theory of Conjugated Systems, (W. A. Benjamin, Inc., New York,1966) p.466

86 R. K. Dodd, J. C. Eilbeck, J. D. Gibbon, H. C. Morris, Solitons and Nonlinear Wave Equations, (Academic Press, London, 1982) p.1

87 W. P. Su, J. R. Schrieffer, A. J. Heeger, J. Phys. Rev. Lett. 42(25), 1698 (1979)

88 W. P. Su, J. R. Schrieffer, Proc. Natl. Acad. Sci. 77(10), 5626 (1980)

89 D. S. Boudreaux, R. R. Chance, J. L. Bredas, R. Silbey, Phys. Rev. B28(12), 6927 (1983)

90 W. P. Su, J. R. Schrieffer, A. J. Heeger, J. Phys. Rev. B22(4), 2099 (1980)b

91 W. Forner, C. L. Wang, F. Martino, J. Ladik, Phys. Rev. B37(9), 4567(1988)

92 H. Takayama, Y. R. Lin-Liu, K. Kaki, Phys. Rev. B21, 2388 (1980)

93 J. C. W. Chien, Polyacetylene Chemistry, Physics, and Material Science, (Academic Press: Orlando, 1984) p.472

94 D. K. Campbell, A. R. Bishop, K. Fesser, Phys. Rev. B26(12), 6862 (1982)

95 B. Horowitz, Solid State Commun. 41,729 (1982)

96 W. P. Su, Phys. Rev. B35(17), 2907 (1987)

97 S. Kivelson, W.-K. Wu, Phys. Rev. B34(8), 5423 (1986)

98 M. Tanaka, H. Fujimoto, K. Kamiya, J. Tanaka, Chem. Lett. 393 (1983)

99 C. K. Chiang, C. R. Fincher Jr., Y. W. Park, A. J. Heeger, H. Shirakawa, E. J. Lewis, S. C. Gau, A. G. MacDiarmid, Phys. Rev. Lett. 39, 1098 (1977)

100 H. Takeuchi, Y. Furakawa, I. Harada, H. J. Shirakawa, J. Chem. Phys. 80(6), 2925 (1984)

101 H. Kuhn, J. Chem. Phys., 16, 840 (1948)

102 M. Aldissi, F. Schue, L. Giral, M. Rolland, Polymer, 23, 246 (1982) 


\section{Chapter II Solid-State Acetylene}

\section{A Solid-State Polymerization}

As discussed in Chapter I, polyacetylene samples are typically very disordered and intractable. More ordered samples possibly prepared by the solid-state polymerization of acetylene offer great promise for helping to answer some long-standing questions about the properties of polyacetylene. Two main advantages to solid-state polymerization are that the polymer usually retains the high degree of order in the monomer crystal and that there are no catalytic residues, when the polymerization is photoinitiated.

Solid-state polymerizations have been induced in a great variety of molecules thermally, and by photolysis, radiation treatment ${ }^{1}$ and the application of pressure. ${ }^{2}$ Radiation treatment is in general more effective than UV photolysis in initiating solid-state polymerizations. Many solids scatter UV light, preventing it from penetrating the sample and being absorbed. ${ }^{1}$ It has been observed that in the solid-state reactions occur via excited-state mechanisms more often than by radical mechanisms. ${ }^{1}$ In some cases, the alignment of the monomer molecules in their crystal structure has been observed to increase their reactivity. ${ }^{3}$ Similarly, unfavorable alignment can hamper or prevent polymerization. In many cases monomer reactivity is increased near solid-solid phase transitions where there is increased molecular mobility. 1

Phenyiacetylene, $\mathbf{l}$ and substituted diacetylenes ${ }^{4}$ are among the many molecules reported to have been polymerized both thermally and photolytically in the solid state. The fact that these unsaturated molecules are susceptible to solid-state polymerization suggests that the photopolymerization of solid acetylene may be possible.

\section{B Acetylene Crystal Structures}

Acetylene has two crystalline phases. The high temperature phase is stable between 190 and $133 \mathrm{~K}$. It is cubic with four molecules per unit cell. 5 A three-dimensional model of this crystal structure was constructed and examined. The molecular alignment in this crystal does not appear compatible with the formation of either polyacetylene isomer.

The low temperature acetylene crystal structure is orthorhombic with two molecules per unit cell. In this crystal structure all the molecules lie in layered planes. ${ }^{6}$ Within the layers each molecule is hydrogen bonded to the triple bonds of two other acetylene molecules (Figure II-1). The layers are $5.551 \AA$ apart and offset by one-half unit cell along the $b$ axis. Despite the large molecular separation there is reason to hope that $a$ trans acetylene polymer could be formed from this nionomer crystal. 



\section{Excited States of Acetylene}

In its ground ${ }^{1} \Sigma_{g}{ }^{-4}$ state, acetylene is a linear $D_{\infty h}$ molecule. The lowest possible $\pi^{*} \leftarrow \pi$ electronic transition in acetylene causes the molecule to distort into either a cis $C_{2 v}$ or trans $\mathrm{C}_{2 \mathrm{~h}}$ geometry. This distortion is driven by the difference in energy between the $\pi_{\mathrm{g}}$ orbital of the linear molecule occupied by the promoted electron and the $3 \mathrm{~b}_{2}$ and $4 \mathrm{ag}_{\mathrm{g}}$ orbitals to which this orbital is correlated in the cis and trans geometries, respectively (Figure II-2). The $\pi_{\mathrm{u}}$ orbital in the linear molecule from which an electron is promoted is correlated to either the $1 b_{1}$ or $4 a_{1}$ orbitals of the cis form, or the $1 b_{g}$ or $1 a_{d}$ orbitals of the trans form. Thus, the symmerries of acetylene's lowest available electronic states are $A_{2}$ and $\mathrm{B}_{2}$ bent cis, and $\mathrm{A}_{\mathrm{U}}$ and $\mathrm{B}_{\mathrm{u}}$ bent trans.

The lowest energy electronic transition observed in acetylene has a weak onset near $237 \mathrm{~nm}$, or $42,199 \mathrm{~cm}^{-1}$. This absorption has a wealth of vibrational and rotational structure which has been analyzed in detail.7,8 The transition has been assigned to excitation into the ${ }^{1} \mathrm{~A}_{\mathbf{u}}$ trans state. Foo and Innes ${ }^{9}$ were able to discem the $0-0$ peak of a second electronic transition amongst the absorptions of the ${ }^{1} \mathrm{~A}_{\mathrm{U}}$ state, by a discontinuity in the ${ }^{13} \mathrm{C}$ isotope shift at $185 \mathrm{~nm}$, or $54,116 \mathrm{~cm}^{-1}$. The transition is observed to have a nine band progression in a $720 \mathrm{~cm}^{-1}$ vibrational mode and has been assigned to the transition to the excited ${ }^{1} B_{u}$ trans state. These two singlet states, ${ }^{1} A_{u}$ and ${ }^{1} B_{u}$, are the only states of the four possible singlet and four corresponding triplet states expected for the $\pi^{*} \leftarrow \pi$ electronic transition which have been observed in direct absorption spectroscopy.

It is not surprising that the triplet states of acetylene have not been observed since the spin selection rules forbidding these transitions should be strictly adhered to in such a small molecule. The four triplet states of acetylene; ${ }^{3} \mathrm{~B}_{2}$ (cis) ${ }^{3} \mathrm{~B}_{\mathrm{u}}$ (trans), ${ }^{3} \mathrm{~A}_{\mathrm{u}}$ (trans), and ${ }^{3} \mathrm{~A}_{2}$ (cis), are predicted ${ }^{10}$ to be located $28,100 \mathrm{~cm}^{-1}(356 \mathrm{~nm}), 31,000 \mathrm{~cm}^{-1}(323 \mathrm{~nm})$, $33,700 \mathrm{~cm}^{-1}(297 \mathrm{~nm})$, and $38,500 \mathrm{~cm}^{-1}(260 \mathrm{~nm})$, respectively, above the ground state. The near-IR electronic absorption spectrum of the transition between the ${ }^{3} \mathrm{~B}_{2}$ (cis), prepared by mercury photosensitization, and the ${ }^{3} \mathrm{~A}_{2}$ (cis) states of acetylene has been observed. ${ }^{11}$ The rotational and vibrational structure in this spectrum allowed the determinat $=n$ of the geometries of both states, found to be in close agreement with the predicted geometries. The separation of the $0-0$ bands is $7,400 \mathrm{~cm}^{-1}$, somewhat less than the predicted separation, $10,400 \mathrm{~cm}^{-1}$. The fact that the lower state was prepared by mercury photosensitization purs its electronic energy below $39,400 \mathrm{~cm}^{-1}$.

The best experimental determination for the energy of an electronic transition to a triplet state of acetylene was obtained with low-energy electron-impact spectroscopy. 12,13 A peak at $5.2 \mathrm{eV},(238 \mathrm{~nm})$ with an onset at $4.5 \mathrm{eV},(276 \mathrm{~nm})$, was identified as a triplet absorption because it showed a weaker dependence on scattering angle than known singlets 


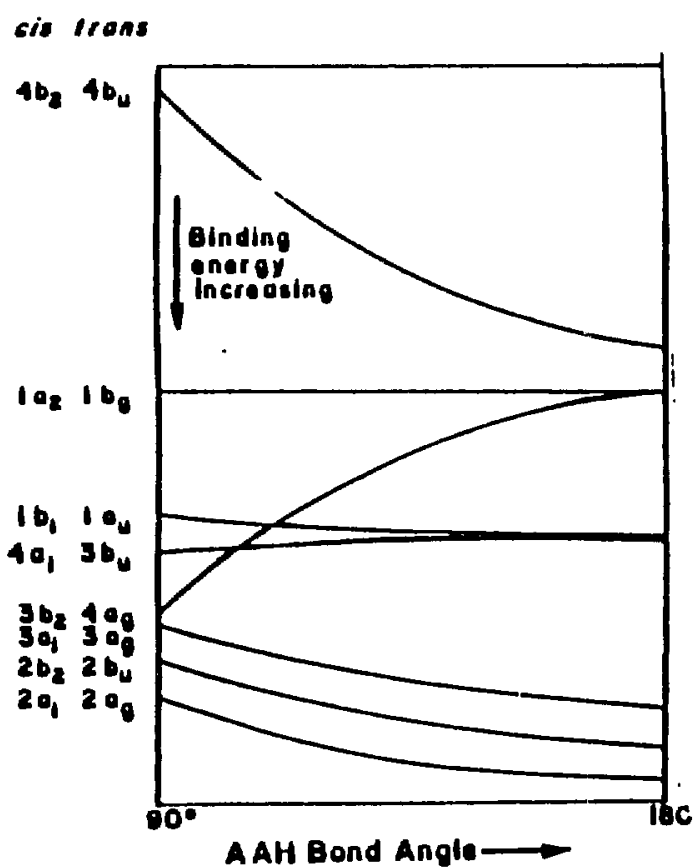

(A)

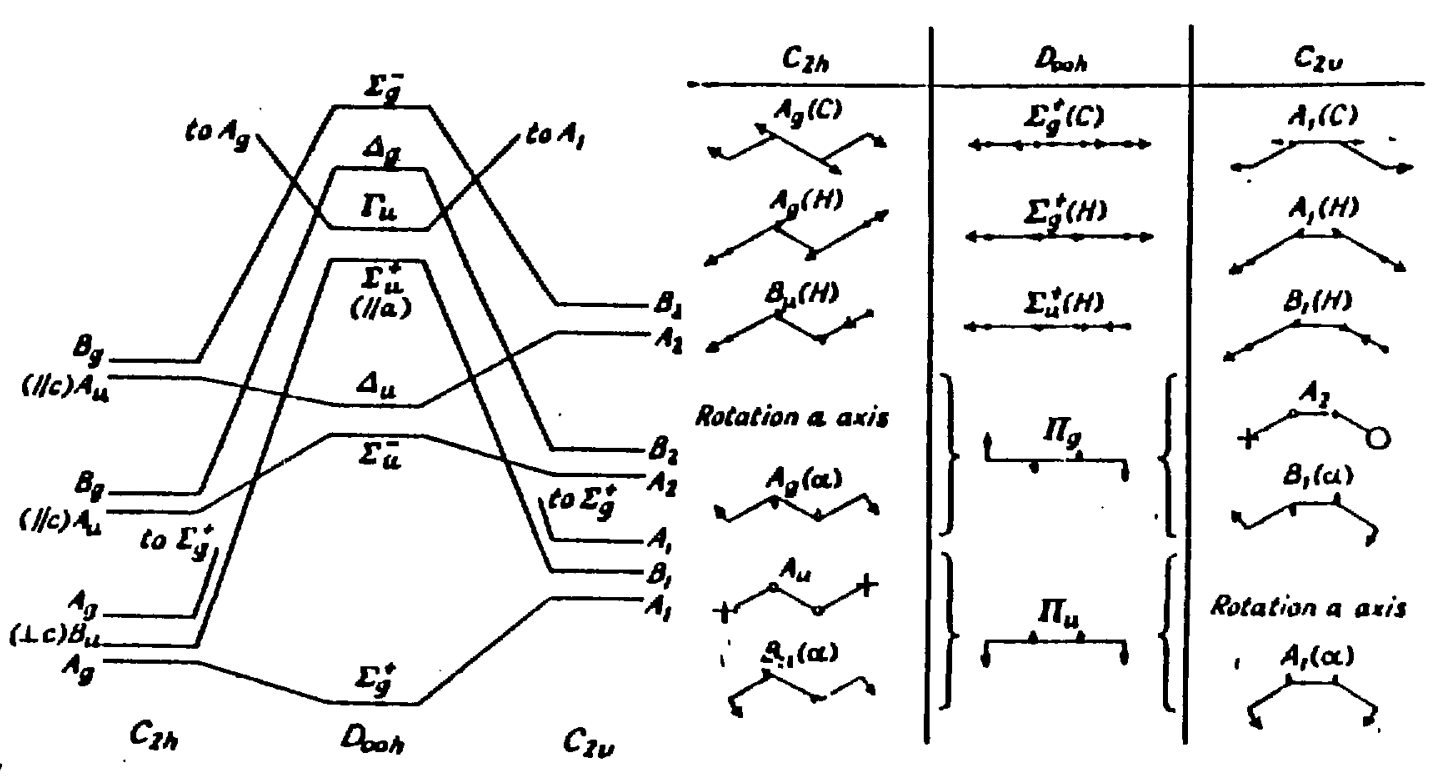

(B)

Figure II-2 Symmetry Considerations for Acetylene

A) The Walsh diagram for acetylene ${ }^{10}$

B) The correlation diagram for the lower electronic states of acetylene 7

C) The symmetries of the vibrations of acetylene in the trans, linear, and cis conformations 7 
and by its location. This absorption was observed with less than $800 \mathrm{~cm}^{-1}$ resolution, no vibrational structure was resolved.

\section{Photochemistry of Acetylene}

The irradiation of acetylene at 185 or $193 \mathrm{~nm}$ excites acetylene to a singlet state. The photoproducts observed for gas phase acetylene photoexcited to singlet states are hydrogen, $14,15,16$ ethylene, 17,15 benzene, ${ }^{18,15}$ diacetylene, ${ }^{18,15,16,17}$ vinylacetylene, 15 and cuprene. $18,15,17$ a crosslinked aromatic polymer of acetylene mentioned in Chapter I. Free radical mechanisms have been proposed for the formation of hydrogen, $14,15,16$ diacetylene, 18,15,16 and cuprene. ${ }^{18,15}$ Mechanisms involving excited-state molecular chemistry have been proposed for the formation of hydrogen, 17 ethylene, 17 benzene, 16,15 diacetylene, 17 vinylacetylene, ${ }^{15}$ and cuprene. ${ }^{18,15}$ The formation of ethylene, benzene, vinylacetylene, diacetylene, and cuprene have been observed during the photoexcitation of

solid acetylene at $110 \mathrm{~K} .19$ Excited state mechanisms are proposed for all the photochernistry observed in the solid state.

The products formed by acetylene excited to a triplet state by mercury photosensitization are essentially the same and the same mixture of mechanisms are proposed. It should be noted that the triplet state of acetylene excited by mercury photosensitization must be at lower energy, $<39,400 \mathrm{~cm}^{-1}$, than the dissociation energy of acetylene. The photodissociation energy of acetylene, $D_{0}=43,000 \mathrm{~cm}^{-1}$, has been determined by time of flight molecular beam measurements. 20 It has been proposed that the dissociation of acetylene at lower energies can be achieved by the formation of a mercury-ethynyl complex. ${ }^{17}$

\section{E.1 Obtaining Crystalline Samples}

Solid samples of acetylene were obtained by depositing gaseous acetylene onto a cold CsI substrate window. The substrate window was mounted on the cold tip of an Air Products closed-cycle Joule-Thomson helium refrigerator (Displex, model CS202), which could cool the sample to $10 \mathrm{~K}$. Temperatures between 10 and $200 \mathrm{~K}$ were maintained by the use of a $20 \mathrm{~W}$ resistance heater mounted on the cold tip. The temperature was monitored at the cold tip with a iron-doped gold/chromel thermocouple and a hydrogen vapor bulb.

The displex as mounted in various spectrometers could be rotated such that the sample was either parallel or perpendicular to the spectrometer beam. The substrate window was rotated out of the spectrometer beam for deposition and photolysis of the samples. 
Before deposition the acetylene (Pacific Oxygen Company, 99.5\%) was purified by bubbling through concentrated $\mathrm{H}_{2} \mathrm{SO}_{4}$ to remove acetone, bulb-to-bulb distillation, and several freeze/pump/thaw cycles, and stored in glass gas bulbs. $\mathrm{C}_{2} \mathrm{D}_{2}$ (MSD Isotopes, lot \# $1768 \mathrm{H}, 99.7 \%$ ) was purified by bulb-to-bulb distillation and several freeze/pump/thaw cycles, and stored in a glass gas bulb.

The optimum conditions for depositing crystalline samples of acetylene were determined by trial and error. The best growing conditions were chosen as those which resulted in the least scattering and most structured far-ultraviolet spectra. These conditions were the rapid "pulse" deposition of acetylene at 30 torr from a $2 \mathrm{ml}$ volume ( $3 \mu \mathrm{moles}$ ) onto a $60 \mathrm{~K}$ substrate window. After deposition the window was cooled to $10 \mathrm{~K}$ at $2^{\circ}$ per minute. The observed structure was lost during UV photolysis and on annealing at temperatures above $80 \mathrm{~K}$.

The IR spectra of samples obtained in this manner were recorded with a PerkinElmer 283 double-beam spectrometer. The frequencies of the observed IR absorptions are reported in Table II-1. These frequencies have been corrected to calibration curves determined by comparison of the gas phase absorption frequencies of the following molecules in the specified frequency ranges to standard values: $\mathrm{NH}_{3}, 3,400 \mathrm{~cm}^{-1}$ and 750 $\mathrm{cm}^{-1} ; \mathrm{CH}_{4}, 3,100 \mathrm{~cm}^{-1} ; \mathrm{H}_{2} \mathrm{O}, 1,400 \mathrm{~cm}^{-1}$; and $\mathrm{C}_{2} \mathrm{H}_{2}, 700 \mathrm{~cm}^{-1}$. The reported frequencies are more reminiscent of those previously reported for polycrystalline ${ }^{21}$ than for single crystal acetylene. 22

\section{E.2 Far-Ultraviolet Spectra of Acetylene}

The far-ultraviolet spectra of the solid acetylene samples were recorded over the spectral range 200 to $170 \mathrm{~nm}$, on a Perkin-Elmer 450 near-IR - visible - ultraviolet - farultraviolet double-beam spectrometer. The spectrometer was operated in the ultraviolet and far-ultraviolet with a deuterium source, photomultiplier detectors, and a strong nitrogen purge. The displex was fitted with $\mathrm{CaF}_{2}$ windows to transmit the spectrometer beam. The wavelength calibration of the spectrometer was set on the visible lines of the deuterium lamp. The wavelength accuracy between 170 and $200 \mathrm{~nm}$ was determined to be $0.1 \mathrm{~nm}$ by the comparise of recorded frequencies for gas phase ammonia absorption to standard values. ${ }^{23}$

At first glance the absorptions observed in solid acetylene (Figure II-3) might appear similar to those observed in the gas phase for the transition to the ${ }^{1} \mathrm{~B}_{\mathrm{u}}$ state. The lowest energy peak at $54,290 \mathrm{~cm}^{-1}$ is followed by a series of peaks $695 \mathrm{~cm}^{-1}$ apart. The frequency of the vibrational mode of the excited state molecule which would appear to be responsible for the observed progression would have shifted $25 \mathrm{~cm}^{-1}$ from $720 \mathrm{~cm}^{-1}$ in the 
Table II-1 IR Spectra of Acetylene

This Work Single Crystal ${ }^{22}$ Polycrystalline Gas Phase $^{21} \quad$ Assignment $^{21}$ $10 \mathrm{~K}_{\left(\mathrm{cm}^{-1}\right)} \quad 83 \mathrm{~K}\left(\mathrm{~cm}^{-1}\right) \quad 63 \mathrm{~K}\left(\mathrm{~cm}^{-1}\right)$ Center Frequency $\left(\mathrm{cm}^{-1}\right)$

\begin{tabular}{|c|c|c|c|c|}
\hline $3862 w$ & $3860 \mathrm{~m}$ & $\begin{array}{l}3867 w \\
3863 w \\
3857 w\end{array}$ & 3884 & $v_{3}+v_{4}$ \\
\hline $3431 \mathrm{vw}$ & $3400 \mathrm{~m}$ & 3429 vw & & $v_{1}+v_{T}$ \\
\hline $\begin{array}{l}3331 \mathrm{vw} \\
3282 \mathrm{w}\end{array}$ & $3308 \mathrm{~s}$ & $3331 w$ & 3295 & $v_{2}+v_{4}+v_{5}$ \\
\hline $\begin{array}{l}3231 \text { vs } \\
3237 \text { vs }\end{array}$ & $\begin{array}{l}3220 \text { vs } \\
3177 \mathrm{~m} \text { (sh) } \\
3112 \mathrm{w}(\mathrm{sh})\end{array}$ & $\begin{array}{l}3226 \mathrm{vs} \\
3221 \mathrm{w}(\mathrm{sh})\end{array}$ & 3282 & $v_{3}$ \\
\hline $2550 w$ & $\begin{array}{l}2715 \mathrm{vw} \\
1597 \mathrm{w}(\mathrm{sh}) \\
1523 \mathrm{~m} \\
1490 \mathrm{~m}(\mathrm{sh})\end{array}$ & $2726 \mathrm{vw}$ & 2703 & $v_{2}+v_{5}$ \\
\hline $\begin{array}{l}1420 \mathrm{~m}(\mathrm{sh}) \\
1390 \mathrm{~m}\end{array}$ & $\begin{array}{l}1423 \mathrm{~s}(\mathrm{sh}) \\
1392 \mathrm{~s}\end{array}$ & $\begin{array}{l}1422 \mathrm{~m}(\mathrm{sh}) \\
1390 \mathrm{~s} \\
1377 \mathrm{~m}(\mathrm{sh})\end{array}$ & 1327 & $v_{4}+v_{5}$ \\
\hline $1283 \mathrm{vw}$ & $\begin{array}{l}1297 \mathrm{vw} \\
1282 w \\
1264 w\end{array}$ & $1280 w$ & & $2 v_{4}$ \\
\hline $\begin{array}{l}899 \text { w(bd) } \\
884 \text { w(bd) }\end{array}$ & $870 \mathrm{~s}$ & $871 \mathrm{vw(bd)}$ & & $v_{5}+v_{R}$ \\
\hline $849 w(b d)$ & $843 \mathrm{~s}(\mathrm{sh})$ & $847 \mathrm{vw}(\mathrm{bd})$ & & $v_{5}+v_{R^{\prime}}$ \\
\hline $\begin{array}{l}789 \mathrm{~m} \text { (sh) } \\
773 \mathrm{vw} \\
762 \mathrm{vs} \\
750 \mathrm{~m}(\mathrm{sh}) \\
721 \mathrm{w} \\
714 \mathrm{w}\end{array}$ & $\begin{array}{l}744 \text { vs } \\
726 \mathrm{~m} \text { (sh) } \\
716 \mathrm{vs}\end{array}$ & $\begin{array}{l}769 \text { vs } \\
761 \text { vs } \\
747 \mathrm{~s}\end{array}$ & 729 & $v_{5}$ \\
\hline
\end{tabular}




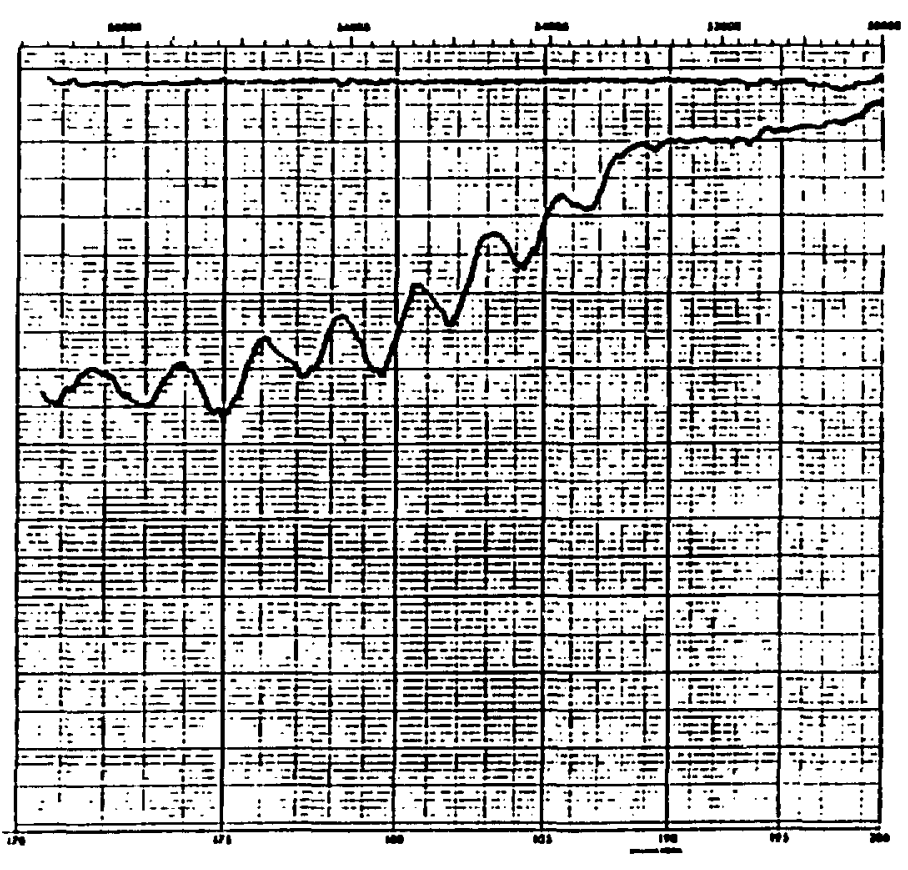

(A)

WAVELENGTI (nm)

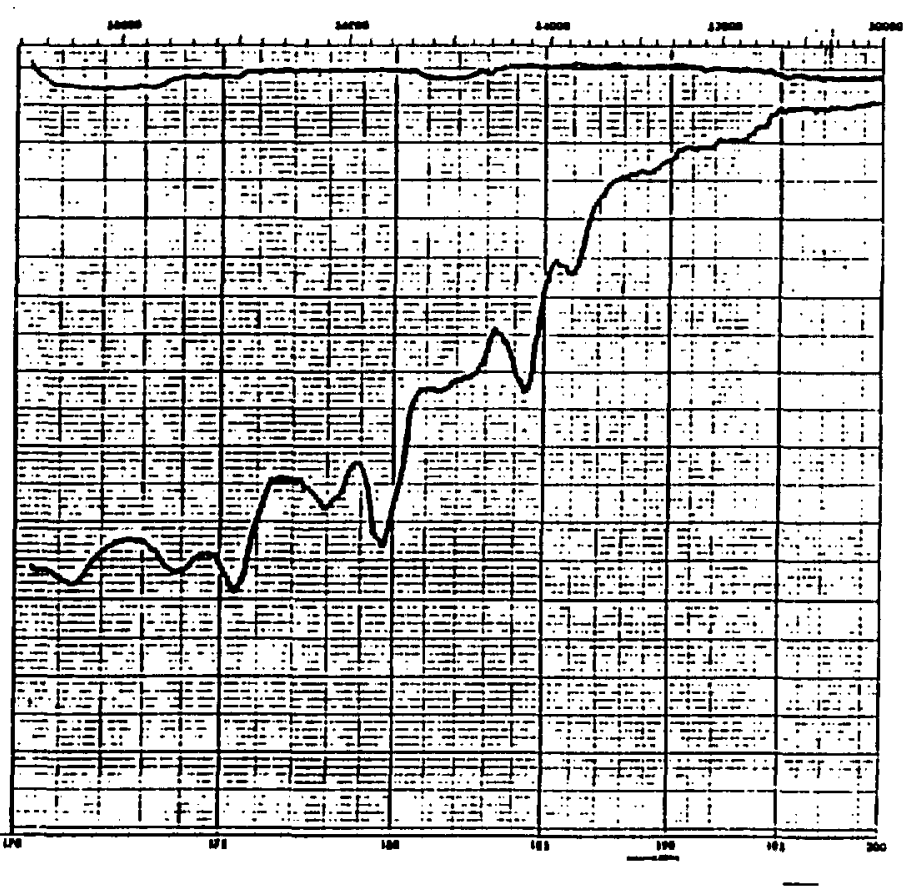

(B)

WAVELENGTH (nm)

Figure II-3 The Far-Ultraviolet Spectra of A) solid $\mathrm{C}_{2} \mathrm{H}_{2}$ B) solid $\mathrm{C}_{2} \mathrm{D}_{2}$ 
gas phase to $695 \mathrm{~cm}^{-1}$ in the solid. This is in keeping with the large shift observed in the IR between the frequency of the $\mathrm{C}-\mathrm{H}$ bending motion of ground state acetylene between the gas and solid phases (Table II-1). However, the spectrum of solid $\mathrm{C}_{2} \mathrm{D}_{2}$ makes it clear that there are really two $1390 \mathrm{~cm}^{-1}\left(1360 \mathrm{~cm}^{-1}\right.$ for $\left.\mathrm{C}_{2} \mathrm{D}_{2}\right)$ progressions offset by one quantum in a $690 \mathrm{~cm}^{-1}\left(530 \mathrm{~cm}^{-1}\right.$ for $\left.C_{2} D_{2}\right)$ vibration (Table II-2). The low energy absorption onset, near $51,500 \mathrm{~cm}^{-1}$, is more evident in the reported spectrum of solid $\mathrm{C}_{2} \mathrm{D}_{2}$. The observed vibrational progressions are probably due to the same vibrations as those observed in the gas phase transition to the ${ }^{1} \mathrm{~A}_{\mathrm{u}}$ excited state, $1389 \mathrm{~cm}^{-1}$ and $1047 \mathrm{~cm}^{-1} .8$ The 0 - 0 band of the gas phase transition to the ${ }^{1} A_{u}$ state is at $42,198 \mathrm{~cm}^{-1}$. The assumed $0-0$ band in the solid is at much higher energy, near $51,570 \mathrm{~cm}^{-1}$. A broad featureless absorption to the ${ }^{1} B_{\mathbf{u}}$ state can be envisioned under the observed progression, with an onset near $53,190 \mathrm{~cm}^{-1}$.

The relevance of these spectra is simply that the samples of solid acetylene were prepared in such a manner that they could be penetrated by UV light at high enough energies to be absorbed by electronic transitions of the acetylene molecules.

\section{E.3 Attempts to Polymerize Solid Acếylene}

Solid samples of acetylene held at $10 \mathrm{~K}, 77 \mathrm{~K}$, and at $133 \mathrm{~K}$, just below the solidsolid phase transition were photolyzed in an attempt to initiate polymerization. A medium pressure mercury lamp, xenon discharge lamp and the spectrometer source in the PerkinElmer $\mathbf{4 5 0}$ spectrometer were used as photolysis sources. The displex mounted in the IR spectrometer during these experiments, except where the UV spectrometer was used as a photolysis source. The IR spectra of the samples before and after photolysis were the same indicating that photopolymerization was not initiated under any of these conditions.

In separate experiments $\mathrm{HBr}, \mathrm{Br}_{2}, \mathrm{Cl}_{2}, \mathrm{I}_{2}, \mathrm{H}_{2} \mathrm{~S}$, and 2,2'-azo-bis(2methylpropionitrile), a common thermal initiator, and less commonly a photoinitiator of free radical organic reactions, were deposited on top of the solid acetylene samples. No sign of polymerization was seen in the comparison of IR spectra taken before and after the photolysis these intended initiators with a medium pressure mercury lamp.

\section{E.4 Other Molecules}

The solid-state polymerization of several other molecules which could result in polyacetylene or polyacetylene-like polymers was also attempted. The molecular alignment in the high temperature crystal structure of dimethlyacetylene appears more conducive to the polymerization of a trans polymer than any of the other crystal structures examined. This crystal structure consists of layered planes. Within each layer the molecules are lined up 
Table II-2 Far-Ultraviolet Spectra of Acetylene and Deuterated Acetylene

$\mathrm{C}_{2} \mathrm{H}_{2}$

\begin{tabular}{llcc} 
Observed Frequency $\left(\mathrm{cm}^{-1}\right)$ & $\mathrm{n}$ & $\begin{array}{c}51,570+ \\
\mathrm{n}(1390)\end{array}$ & $\begin{array}{c}51,570+ \\
690+\mathrm{n}(1390)\end{array}$ \\
\hline & 1 & 52,960 & 53,650 \\
54,290 & 1 & & \\
55,040 & 2 & 54,350 & 55,040 \\
55,730 & 2 & & \\
56,430 & 3 & 55,740 & 56,430 \\
57,160 & 3 & & \\
57,840 & 4 & 57,130 & 57,820 \\
58,530 & 4 & &
\end{tabular}

$\mathrm{C}_{2} \mathrm{D}_{2}$

$\begin{array}{llcc} & & 51,570+ & 51,570+ \\ \text { Observed Frequency }\left(\mathrm{cm}^{-1}\right) & \text { in } & \mathrm{n}(1360) & 530+\mathrm{n}(1360)\end{array}$

1

$5 \overline{2,930}$

53,760

53,460

54.290

$54,980 \quad 2$

54,820

$55,680 \quad 355,650$

$56,150 \quad 3$

56,180

57,010

57,010

$57,540 \quad 4$

58,380 
end to end in rows. These rows are offset half the distance required along the row for each molecule and are close enough to each other to react readily. Unfortunately this structure is only stable above $154 \mathrm{~K}$. Dimethylacetylene sublimes off the substrate window at temperatures this high. In the low temperature dimethylacetylene crystal, the molecules are tilted out of the plane in alternate directions creating a slightly less favorable geometry for polymerization. The UV photolysis of dimethylacetylene in the low temperature phase was unsuccessful at initiating polymerization. Solid dimethylacetylene samples were doped up to $10 \%$ with xenon. It was hoped that this would allow better access to the long-lived triplet states. Xenon is believed to facilitate intersystem crossing in its neighbors. ${ }^{24}$ Photolysis of these doped samples did not initiate polymerization either.

The head-to-tail polymerization of 1,5-hexadiyne, accompanied by hydrogen migration, would produce polyacetylene. The molecules in both the high and low temperature crystal structures of 1,5-hexadiyne are reported to be in their anti form. 25 Thus polymerization of 1,5-hexadiyne might be expected to produce trans polyacetylene. Such a polymerization was not initiated by the UV photolysis of 1,5-hexadiyne.

Phenylacetylene has been reported to polymerize in the solid state thermally. 1 Attempts were made to photopolymerize samples of phenylacetylerie sublimed onto the substrate window and held at $10 \mathrm{~K}$. IR spectra taken before and after photolysis showed no evidence of polymerization. However, when the bulk of the sample was removed, by warming the substrate window until the sample sublimed into a dynamic vacuum maintained in the displex, a thin polymer film remained on the substrate window. The $\mathbb{R}$ spectra of these films indicated a crosslinked or branched polymer. The acetylenic $\mathrm{C}-\mathrm{H}$ streiching frequency at $3,280 \mathrm{~cm}^{-1}$, in the monomer was replaced by a variety of absorption peaks between 2972 and $2926 \mathrm{~cm}^{-1}$, in the saturated C-H stretching region. This polymer may have been formed either during photolysis or warm-up.

\section{F Search for Triplet Acetylene}

The excitation of acetylene into its triplet states could be more effective at initiating the polymerization of solid acetylene than excitation into the singlet states. The triplet states of acetylene should have very long lifetimes because of the same spin selection rules which cause them to have such smail absorption cross-sections. The long lifetimes would allow more time for reaction. The triplet states may also be more likely to promote molecular excited-state chemistry, typically more effective in the solid state than free radical reaction pathways. Transitions from the ground to the triplet states of acetylene have never been observed in direct absorption. These transitior.: $\mathrm{i}$ ust, however, have some finite absorption. If the exact locations were known, 
certainly excite a few molecules. Only a few molecules would need to be excited to initiate polymerization.

Very weak electronic absorptions have been detected indirectly by monitoring the rate of a facile reaction involving the excited molecules as a function of photolysis wavelength. 26 The resultant reaction excitation spectrum is a convolution of the absorption of the transition excited and the reaction probability for the geometry and energy of the specific excited state. In a narrow excitation energy range the reaction excitation spectrum should directly reflect the spectrum of the absorption in question.

An attempt was made to determine the exact energy of the transitions to the triplet states of acetylene from reaction excitation spectra of the photoinduced reaction between acetylene and oxygen isolated in an argon matrix. Oxygen was selected as the reaction partner because it has a triplet ground state, and its presence might relax the selection rules in the acetylene. Isotopically labeled ${ }^{13} \mathrm{C}_{2} \mathrm{H}_{2}$ (Merk Sharp and Dome; acetylene $1,2{ }^{13} \mathrm{C}_{2}$ $99 \%$ lot \#1973J) was used so that the reaction product ${ }^{13} \mathrm{CO}_{2}$ could be distinguished from impurity $\mathrm{CO}_{2}$. The matrix isolation set up and IBM IR/97 spectrometer used are described elsewhere. ${ }^{24}$ A $1: 5: 200$ mixture of ${ }^{13} \mathrm{C}_{2} \mathrm{H}_{2}: \mathrm{O}_{2}$ : Ar was used. With this ratio, assuming no preferences, $70 \%$ of the acetylene molecules should be isolated, $21 \%$ of the acetylene molecules should have one oxygen nearest neighbor, and $<5 \%$ should.have more than one nearest neighbor other than argon.

Preliminary experiments using a high pressure xenon/mercury arc lamp and interference filters as the photolysis source showed an onset for the production of ${ }^{13} \mathrm{CO}_{2}$ between 323 and $296 \mathrm{~nm}$. Greater photolysis wavelength selectivity was obtained by using a doubled tunable dye laser as the photolysis source. The output of a Quanta-Ray YAG laser (DCR-2) was doubled with a KD*P crystal in a Quanta-Ray harmonic generator (HG2). The doubled YAG beam was separated from the fundamental in a Quanta-Ray prism harmonic separator (PHS-1) and used to pump a Quanta-Ray flowcell dye laser (PDL-1). The dye laser output was doubled with another $\mathrm{KD} * \mathrm{P}$ crystal in a Quanta-Ray wavelength extension system (WEX-1A). The doubled dye output was directed onto the matrix with a series of prisms. The system was run at $10 \mathrm{~Hz}$ with at a typical power output of $2-10 \mathrm{~mJ}$ per pulse between 285 and $275 \mathrm{~nm}$ using doubled Rhodimine 590 .

The experiment was repeated with deuterated ${ }^{13} \mathrm{C}$ acetylene (Cambridge Isotope Labs; acetylene $1,2{ }^{13} \mathrm{C} 1,2 \mathrm{D}_{2}\left({ }^{13} \mathrm{C} 99 \% ; \mathrm{D}_{2} 99 \%\right)$ lot F-7294). The possibility that the observed reaction was caused by oxygen or oxygen dimer absorption could be eliminated if the absorption had vibrational structure which showed a shift when deuterated acetylene was used.

The excitation spectra, ${ }^{13} \mathrm{CO}_{2}$ productior as a function of the photolysis frequency, 
for several runs are shown in Figure II-4. In each spectrum, the reaction efficiencies for a few frequencies were recorded several times. The error bars reflect the greatest standard deviation in the observed reaction efficiencies at a frequency where more than one measurement was taken. No systematic variation in the reaction rate, vibrational structure, was observed above the noise of the experiment or reproducibly. Thus it is not possible to attribute the reaction to a specific absorption. The poor reproducibility is attributed to difficulties obtaining the same the photolysis beam shape and realigning the laser beam on the sample after changing the laser frequency.

\section{G Insurmountable Difficulties}

The factors governing the feasibility of solid-state photopolymerization are penetration of exciting light into sample, lifetime of excited species, and proper alignment of molecules in a reactive geometry. It has been demonstrated that light capable of exciting acetylene to a singlet excited state was able to penetrate the samples. The lifetime of the reactive species, photoexcited molecules, might have been increased by excitation of the triplet: unfortunately, the location of these states remains unknown. There is considerable separation between the carbons to be joined by polymerization in both the high and low temperature crystal structures of acetylene. It appears probable that acetylene cannot be polymerized to linear polyacetylene in the low temperature crystal structure because the molecules are too far apart. 


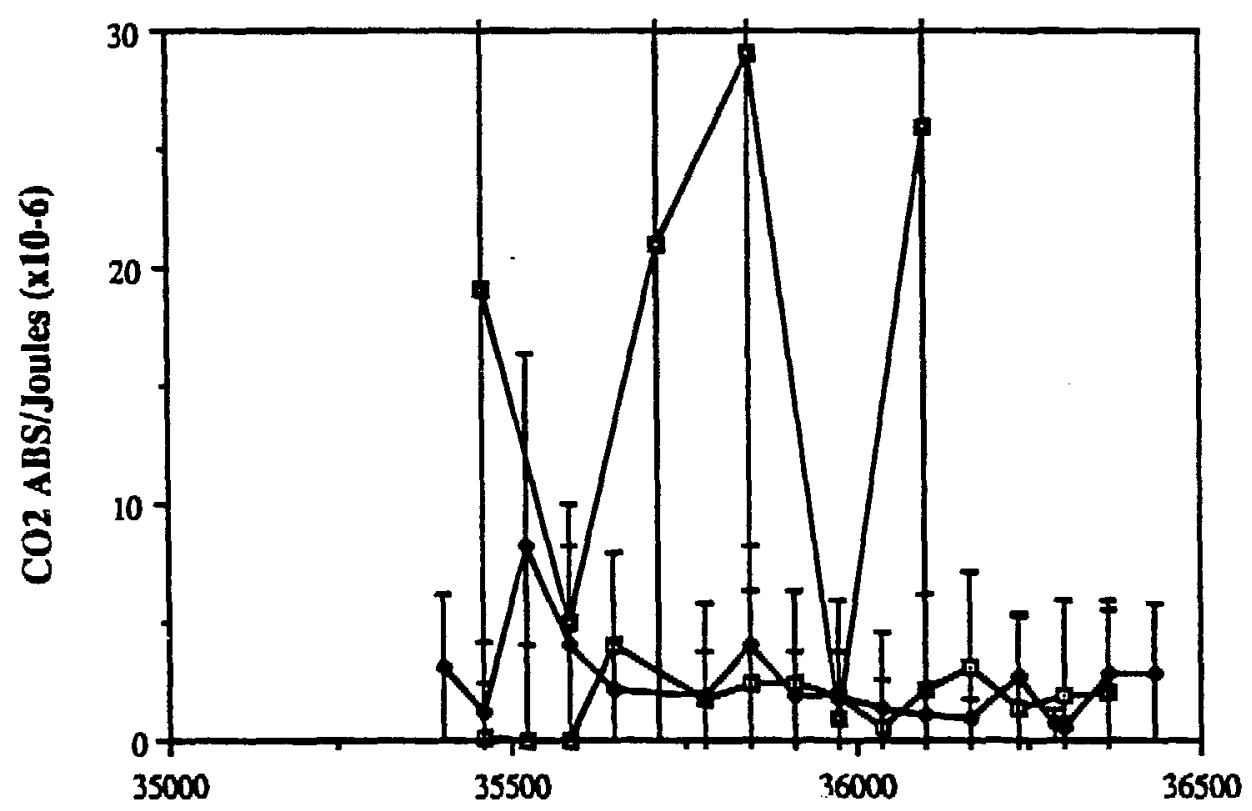

(A)

Photolysis Frequency (Wavenumbers)

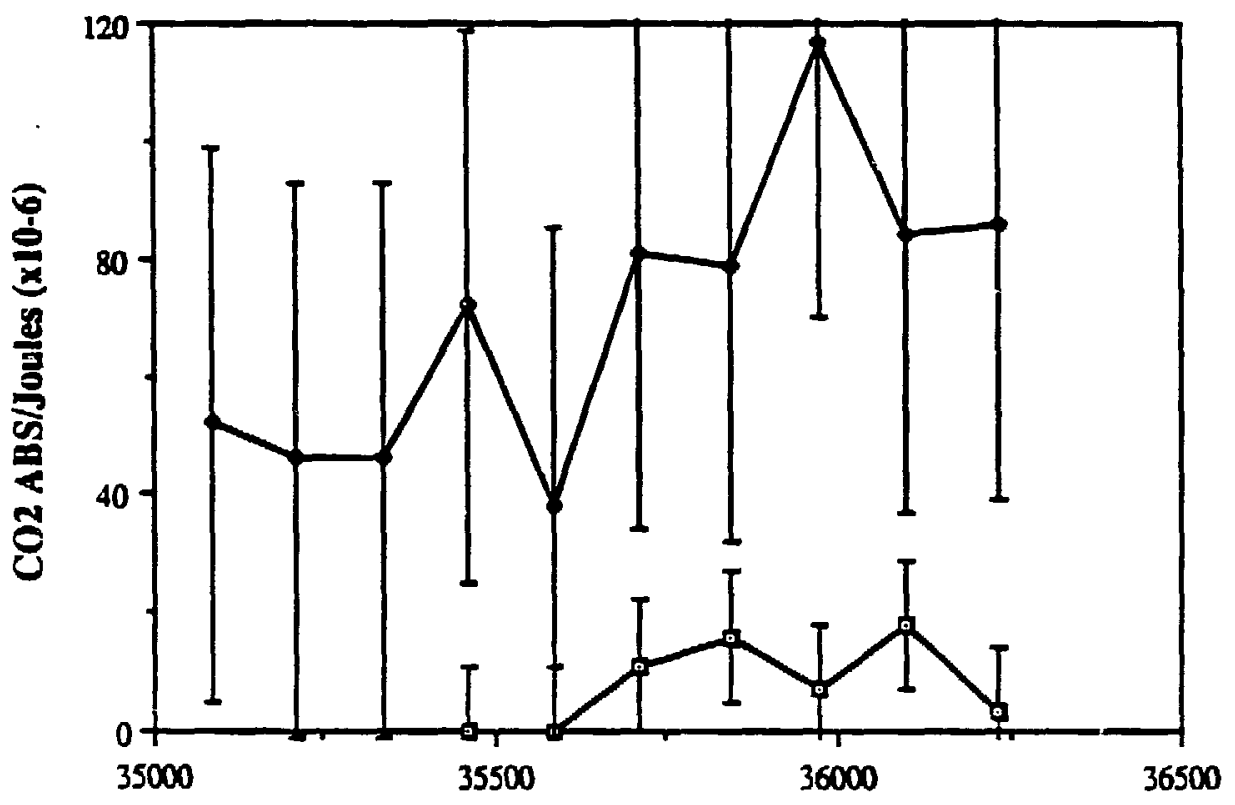

(B)

Photolysis Frequency (Wavenumber)

Figure $\amalg-4$ Reaction Excitarion Spectra

Absorbance at $13 \mathrm{~cm}^{-1}$ of ${ }^{13} \mathrm{CO}_{2}$ produced per Joule during the phorolysis of a $1: 5$ : 200 marrix of A) ${ }^{13} \mathrm{C}_{2} \mathrm{H}_{2}: \mathrm{O}_{2}:$ Ar B) ${ }^{13} \mathrm{C}_{2} \mathrm{D}_{2}: \mathrm{O}_{2}: \mathrm{Ar}$ 


\section{References}

1 G. B. Sergeev, V. A. Batyak, Cryochemistry; trans. B. V. Kuzetov; Mir Publishers, Moscow, 1981

2 C.-S. Yoo, M. Nicol, J. Phys. Chem. 90, 6726 (1986)

3 R. B. Wilson, Y.-S. Chen, 1. C. Paul, D. Y. Curtin, J. Am. Chem. Soc. 105,1672 (1983)

4 G. Wegner, Die. Mackromolekulare Chemie. 154, 35 (1972)

5 R. W. G. Wykoff, Crystal Structures, 2ed. vol.5 (Interscience Publishers, New York, 1969) p. 265

6 H. K. Koski, E. Sandor, Acta Cryst. B31, 350 (1975)

7 C. K. Ingold, G. W. King, J. Chem Soc. 2702 and 2708, (1953)

8 K. K. Innes, J. Chem Phys. 22(5) 863, (1954)

9 P. D. Foo, K. K. Innes, Chem Phys. Lett. 22(3) 439, (1973)

10 R. W. Wetmore, H. F. Schaefer III, J. Chem. Phys. 69(4), 1648 (1978)

11 H. R. Wendt, H. Hippler, H. E. Hunzinker, J. Chem. Phys. 70(9), 4044 (1979)

12 S. Trajmar, J. K. Rice, P. \& P. Wei, A. Kuppermann, Chem. Phys. Letts. 1, 703 (1968)

13 W. M. Flicker, O. A. Mosher, A. Kuppermann, J. Chem. Phys. 68(7), 3311 (1978)

14 S. Shida, Z. Kuri, T. Furuoya, J. Chem. Phys. 28(1), 131 (1958)

15 M. Zelikoff, L. M. Aschenbrand, J. Chem. Phys. 24(5), 1034 (1956)

16 H. Okabe, J. Chem Phys. 78(3), 1312 (1983)

17 M. P. Irion, K. L. Kompa, Appl. Phys. B27, 183 (1982)

18 M. Tsukada, S. Shida, Bull. Chem. Soc. Jpn. 43, 3631 (1970)

19 T. Okuyama, N. Nishi, Ann. Rev. Inst. Mol. Sci. 72, (1983)

20 A. Wodtke, Y. T. Lee, J. Phys. Chem. 89, 4744 (1985)

21 G. L. Bottger, D. F. Eggers, Jr, J. Chem. Phys. 40(7), 2010 (1964)

22 E. Kirkorian, Ph. D. dissertation, Columbia University, 1957

23 A. B. F. Duncan, Phys. Rev. 47, 822 (1935)

24 S. T. Collins, G. C. Pimentel, J. Phys. Chem. 88, 4258 (1984) 
25 G. O. Braathen, C. J. Nielsen, P. Klaeboe, H. Hopf, J. Mol. Struct. 74, 233 (1981)

26 H. Frei, J. Chem. Phys. 80(11), 5616 (1984) 


\section{Chapter III Experimental}

\section{A Sample Preparation}

Two syntheses were used for the preparation of samples used in these studies. The Shirakawa method ${ }^{l}$ was used to produce free-standing thin films. These films ranged in thickness from 20 to $220 \mu \mathrm{m}$ and were suitable for electrical resistance measurements, infrared and near-IR spectroscopy. The popular Shirakawa method was not used exclusively because of difficulties in maintaining good contact with the substrate windows, which is required for proper temperature control.

The Baker synthesis, ${ }^{2}$ also used, produces a colloid of polyacetylene which can be dried to a thin film. These films adhered well to the substrate, making them useful for temperarure dependent work. These samples were, however, not cohesive enough for conductivity measurements, and their thickness could not be varied significantly. The concentration of neutral defects in polyacetylene samples prepared with this synthesis is one tenth that in Shirakawa polyacetylene. The use of both syntheses allows the effects of the neutral defect concentration to be studied.

All polymerization procedures were carried out under vacuum or in a nitrogen environment, either in a dry box or with positive nitrogen pressure. All glassware was dried at $140^{\circ} \mathrm{C}$, cooled in the dry box or under vacuum, and stored in the dry box. Solvents were dried over $\mathrm{Na} /$ benzophenone (toluene and THF) or $\mathrm{CaH}_{2}$ (hexane), distilled under nitrogen, degassed by several freeze/pump/thaw cycles and stored in the dry box. The acetylene (Pacific Oxygen Company, 99.5\%) was bubbled through concentrated $\mathrm{H}_{2} \mathrm{SO}_{4}$ to remove acetone, further purified by bulb-to-bulb distillation and several freeze/pump/thaw cycles, and stored in $2 l$ glass gas bulbs. $\mathrm{C}_{2} \mathrm{D}_{2}$ (MSD Isotopes, lot \# $1768 \mathrm{H}, 99.7 \%$ ) was purified by bulb-to-bulb distillation and several freeze/pump/thaw cycles, and stored in a glass gas bulb. $\mathrm{Ti}(\mathrm{BuO})_{4}(\mathrm{lot} \# 102782)$ and $25 \% \mathrm{Al}(\mathrm{Et})_{3}$ in hexane (lot \# 121378) were obtained from Alfa and used without any further purification. No greased joints were used. Teflon stopcocks were used.

\section{A.1 Shirakawa Polymerization}

Shirakawa polyacetylene was prepared as described in the original preparation, ${ }^{1}$ but with some modifications in experimental set-up. The reaction vessel (Figure III-IA) consisted of a glass cylinder which could be opened at o-ring (b), above which it tapered sharply to a much smaller o-ring joint (a). A glass connector with Teflon stopcock could be used to attach the reaction vessel at o-ring joint (a) to a flexible rubber tube connected to a vacuum line. 


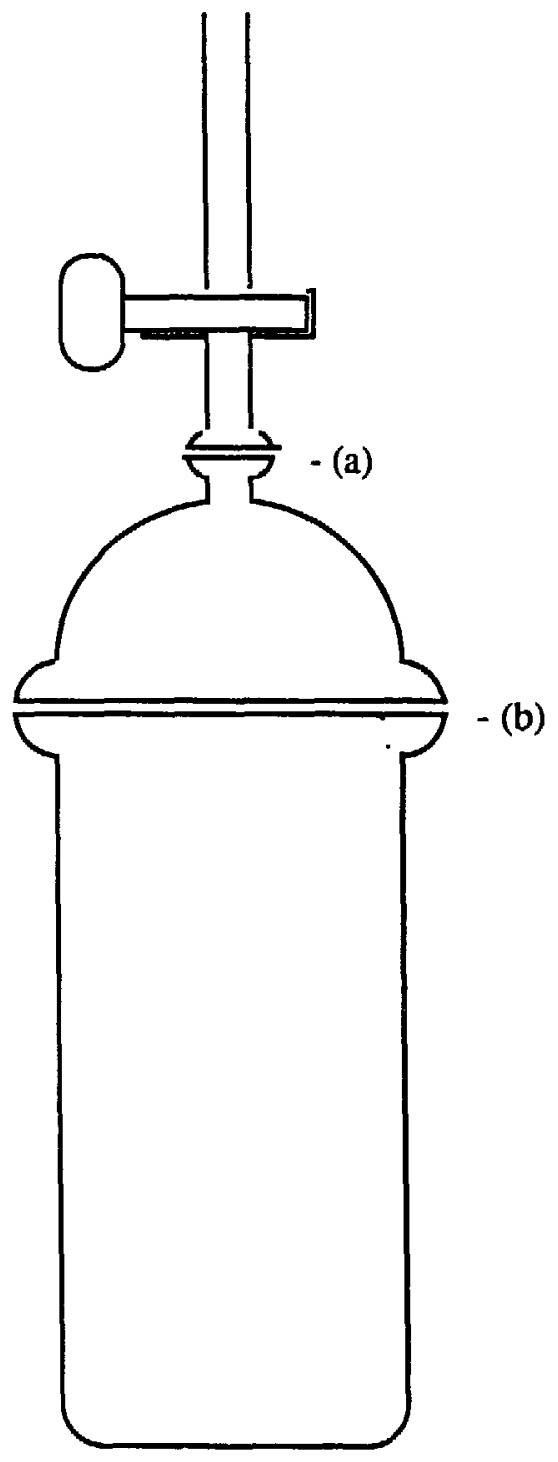

(A)

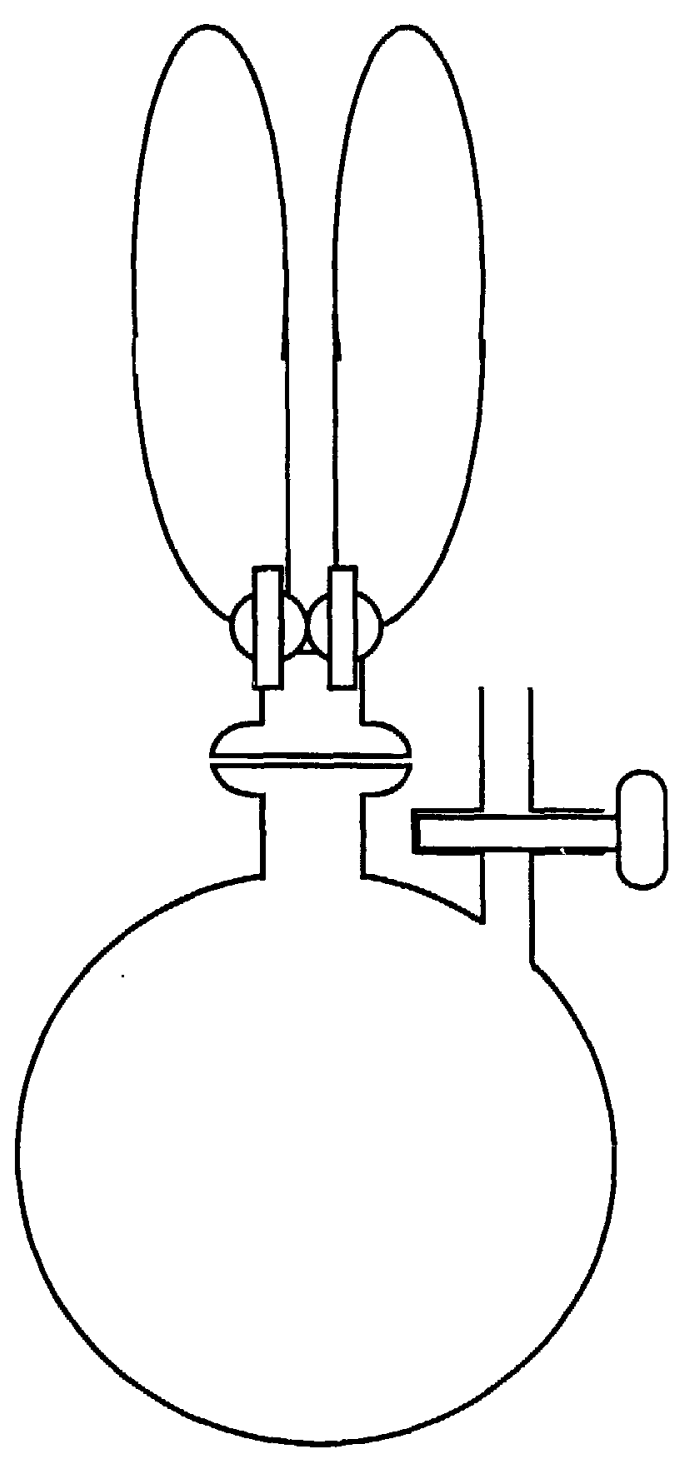

(B)

Figure III-1 Reaction Vessels

A vessel used in Shirakawa polymerization B vessel used in the Baker preparation 
In the dry box, $0.13 \mathrm{ml}(0.38 \mathrm{mM})$ of $\mathrm{Ti}(\mathrm{BuO})_{4}$ and $1.5 \mathrm{ml}$ of toluene were added to the reaction vessel using gas-tight syringes. A septum cap was placed over o-ring joint (a) and a syringe containing $1 \mathrm{ml}$ of $25 \% \mathrm{Al}(\mathrm{Et})_{3}(1.5 \mathrm{mM})$ in hexane was inserted through the cap. The vessel was removed from the dry box and cooled to $195 \mathrm{~K}$ in a dry ice/isopropanol slush bath. After the $\mathrm{Al}(\mathrm{Et})_{3}$ was added, the catalyst solution, $0.56 \mathrm{molar}$ in $\mathrm{Al}$, with a $4: 1 \mathrm{Al}$ : Ti ratio, was allowed to warm up to room temperature and age for half an hour. The reaction vessel was returned to the dry box where the septum cap was replaced with the o-ring to rubber tubing connector. The reaction vessel was again removed from the dry box, connected to the vacuum line and evacuated while being cooled in dry ice/isopropanol. The cold reaction vessel was tilted and swirled to coat its walls below o-ring joint (b) with the catalyst solution. Several hundred torr of acetylene were then admitted, of which typically 25 torr were taken up by polymerization on the walls of the reaction vessel. After the polymerization, the vessel was evacuated, allowed to warm up, and returned to the dry box. The catalyst was removed from the polymer by multiple solvent rinses.

Isomerization was achieved by evacuating the reaction vessel containing the polymer and suspending it up to the o-rings in hot oil $\left(110^{\circ} \mathrm{C}\right)$. The polymer was pried from the walls and cut into 1 " circles which were either wetted to a substrate window or sandwiched between two.

\section{A.2 Baker Polymerization}

The procedure for synthesizing Baker polyacetylene ${ }^{2}$ (b-PA) was also modified somewhat. The reaction vessel used for the preparation of b-PA (affectionately known as the rabbit reactor) (Figure $\mathrm{II}-1 \mathrm{~B}$ ) consisted of two non-equalizing addition funnels connected to a Schlenk flask by an o-ring joint. In the dry box, $10.0 \mathrm{ml}$ of dried, distilled, degassed toluene and $0.24 \mathrm{ml}$ of $\mathrm{Ti}(\mathrm{BuO})_{4}(0.70 \mathrm{mM})$ were mixed in one funnel, $3.59 \mathrm{ml}$ toluene and $0.34 \mathrm{ml}$ of $25 \% \mathrm{Al}(\mathrm{Et})_{3}(0.59 \mathrm{mM})$ in hexanes in the other. The flask was removed from the dry box, connected to a vacuum line by flexible rubber tubing and evacuated. In a typical reaction, 200 torr of acetylene were introduced to the flask, the $\mathrm{Ti}(\mathrm{BuO})_{4}$ mixture was added and stirred. then the $\mathrm{Al}(\mathrm{Et})_{3}$ was added. Thus a catalyst solution, 0.04 molar in $\mathrm{Al}$, with close to a $\mathrm{l}: 1 \mathrm{Al}$ : Ti ratio was formed in the presence of the monomer. The solution was stirred for half an hour while the newly formed catalyst polymerized all the acetylene. The catalyst was exiracted from the polymer suspension by repeated centrifugations with toluene. Several solvents were tried, toluene appeared to be the most effective at removing the catalyst. After all the catalyst appeared to have been washed out, any that remained was removed with toluene in a Soxhlet extractor. This 
procedure heated the sample to $\sim 100^{\circ} \mathrm{C}$, bringing about nearly complete isomerization. The colloidal suspension was dried to thin films directly on the substrate windows. The smoothest films were obtained by the slow evaporation of hexane.

\section{B.1 Sampie Handling}

Attempts were made to minimize the exposure of the polyacetylene samples to air, in particular oxygen and water. However, the samples were exposed to the air for a few minutes while being installed in the Displex sample holder. The near-IR transmission of sample \#2, before and after being installed and removed seven times, is shown in Table III-

2. There was no indication of sample degradation in the $\mathbb{R}$, thus the loss in near- $\mathbb{R}$ transmittance may have been due to increased scatter from changes in the condition of the sample's surface. All samples were stored at room temperature in the dry box. Baker polyacetylene particles were kept in a hexane suspension until they were dried as films.

\section{B.2 Filters and Photolysis Setup}

A General Electric "Chill Chaser" heat lamp was used as a near-IR source. The light was collected with a reflective shade and an aluminum foil cone. It was directed through a filter and onto the sample by 1 " diameter glass tubes lined with Mylar. The lamp required half an hour to warm up to a stable power output. A camera shutter was used for shor, timed photolyses.

The power passed by each filter was measured with a Scientech volume absorbing disc calorimeter (\#38-0101). Spectra of the filters were recorded with an IBM IR/97 fourier-transform spectrometer $\left(400-12,000 \mathrm{~cm}^{-1}\right)$ or a Varian $2300 \mathrm{UV}$-VIS-NIR spectrometer $\left(v>4,000 \mathrm{~cm}^{-1}\right)$. The various filters and filter combinations used, the frequencies transmitted, and typical power throughput are listed in Table III-1. The circular variable filter (CVF) (Optics Coating Laboratory) ${ }^{3}$ was used with extra wide slits $(0.12 \mu)$ to allow for a greater power throughput. Filters listed by number are narrow band pass filters from Optics Coating Laboratory. CG refers to a Corning Glass 2-60 filter, Si to a 1 mm thick silicon plate, $\mathrm{Ge}$ to a $5 \mathrm{~mm}$ thick germanium plate, and $\mathrm{H}_{2} \mathrm{O}$ to a $1^{\text {" water filter. }}$

\section{C.1 Conductivity Measurement}

The resistance of undoped samples was measured with a Keithley 616 Digital Electrometer. A two-point conductivity probe, appropriate for samples with significantly greater resistance than the contacts, was used for undoped samples. The film was sandwiched between two thin salt windows, one of which had been drilled to allow two 
Table III-1 Photolysis Conditions

Filter (Combination)

Frequency Range

$\left(\mathrm{cm}^{-1}\right)$
Typical Throughput (mW)

C V F

2,800

3,000

3,200

3,400

3,600

3,400

4,000

4,200

4,400

4,600

4,800

5,000

5,200

Ge

"5,810" (N01721-7 + Si)

"6,950" (N01431-6 + Si)

$" 8,690 "\left(\mathrm{H}_{2} \mathrm{O}+\mathrm{Si}\right)$

"9,300" (N01076-7 + $\left.\mathrm{H}_{2} \mathrm{O}+\mathrm{CG}\right)$

$" 10,700 "\left(\mathrm{~N} 00947-7 \mathrm{~B}+\mathrm{H}_{2} \mathrm{O}\right)$

$\mathrm{CG}+\mathrm{H}_{2} \mathrm{O}$
$2,754-2,848$

$2,947-3,055$

$3,140 \cdot 3,263$

$3,332-3,471$

$3,523-3,679$

$3,889-3,471$

$3,906-4,098$

$4,097-4,309$

$4,287-4,519$

$4,476-4,731$

$4,666-4,942$

$4,854-5,155$

$5,043-5,367$

$<5,500$

$5,688-5,938$

$6,600-7,300$

$7,576-9,800$

$9,163-9,431$

$10,340-11,100$

$7,576-16,530$
4.17

3.96

3.45

4.12

4.45

5.48

4.91

5.81

$5.8 i$

6.76

6.12

5.57

4.60

285.0

97.7

116.0

20.7

12.6

22.4

203.0 
0.01 " platinum wires to be fed though. Pressure contacts were established between the wires and the polymer film when the windows were clamped in the sample holder.

\section{C.2 Photoconductive Response}

At room temperature, the resistance of Shirakawa polyacetylene sample \#2 was $3.4 \times 10^{9} \Omega$. Since the probes were much closer to each other than the edge of the sample, the sample was treated as an infinite sheet ${ }^{4}$ Thus the samples resistivity was its resistance multiplied by its thickness. This sample was $0.02 \mathrm{~mm}$ thick, thus its conductivity, the reciprocal of the resistivity, was $1.5 \times 10^{-7} \Omega^{-1} \mathrm{~cm}^{-1}$. No significant change in resistance was observed during photolysis with frequencies below $6,000 \mathrm{~cm}^{-1}$. The resistance decreased slightly during photolysis at frequencies above $6,000 \mathrm{~cm}^{-1}$. The change in the resistance increased sharply between $9,000 \mathrm{~cm}^{-1}$ and $11,000 \mathrm{~cm}^{-1}$. The photon flux in the region where a change was observed, between 9,000 and $11,000 \mathrm{~cm}^{-1}$, was from 4 to 20 times greater than below $6,000 \mathrm{~cm}^{-1}$. Thus, a larger photoconductive response than that observed between 9,000 and $11,000 \mathrm{~cm}^{-1}$ would have been required for detection below $6,000 \mathrm{~cm}^{-1}$. The percentages of change in the resistance per $\mathrm{mW}$ of incident radiation for the various filter combinations, along with the samples optical densities at the corresponding photolysis frequencies, are given in Table III-2.

The resistance of the sample increased to $1.7 \times 10^{12} \Omega\left(5.9 \times 10^{-13} \Omega^{-1} \mathrm{~cm}^{-1}\right.$ conductivity) when the sample was cooled to $10 \mathrm{~K}$. This decrease in conductivity has been observed previously. ${ }^{5}$ It is expected in large-gap semiconductors because fewer charge carriers are thermally generated at low temperatures. This effect would be greatly mediated by the presence of dopants, thus a large decrease is indicative of a pure sample. No change was observed in the resistance of the cold sample during photolysis at any frequency. This is consistent with previous reports that the photoconductive response is lost at low temperatures.

\section{D.1 IR setup}

The substrate window and sample were mounted in a sample holder at the end of the cold tip in an Air Products closed-cycle Joule-Thomson helium refrigerator (Displex, model CS202). The Displex could cool the sample to $10 \mathrm{~K}$. Temperatures between 10 and $200 \mathrm{~K}$ could be maintained by the use of a $20 \mathrm{~W}$ resistance heater mounted on the cold tip. The temperature was monitored at the cold ip with a iron-doped gold/chromel thermocouple and a hydrogen vapor bulb. 
Table III-2 Sample (*2) Transmittance and Photoconductive Response

Filter (Combination)
Sample \%T
initial final

2,800

3,000

3,200

3,400

3,600

3,800

4,000

4,200

4,400

4,600

4,800

5,000

5,200

"5,810" (N01721-7 + Si)

"6,950" (N01431-6 + Si)

"8,690" $\left(\mathrm{H}_{2} \mathrm{O}+\mathrm{Si}\right)$

"9,300" (N01076-7 + $\left.\mathrm{H}_{2} \mathrm{O}+\mathrm{C} . \mathrm{G}\right)$

"10,700" (NO0947-7B + $\left.\mathrm{H}_{2} \mathrm{O}\right)$

$\mathrm{CG}+\mathrm{H}_{2} \mathrm{O}$
$\% \Delta \mathrm{R} / \mathrm{mW}$

$-95$

$-93$

$-92$

90

87

84

83

79

77

73

67

64

64

62

67

51

0.002

57

19

0.003

32

1

0.021

23

0

0.035

08

0

0.045

0

0

0.038 
The Displex was mounted in the beam path of a fourier-transform $\mathbb{R}$ spectrometer, either an IBM IR/44 or IBM IR/97. The sample was oriented perpendicular to the spectrometer beam to take spectra or rotated parallel to the beam path for photolysis.

\section{D.2 Doping at Low Temperature; IR and near-IR}

Polyacetylene samples were mounted in the Displex in the IBM IR/97 spectrometer and cooled to $10 \mathrm{~K}$. Dopants introduced at this temperature did not react with the polymer but merely froze to the surface. The temperature ranges in whicl: the dopants began to induce $\mathbb{R}$ and near-IR absorptions were determined by monitoirg the samples' spectra during warming. IR spectra were taken from 4500 to $400 \mathrm{~cm}^{-1}$ at $1.0 \mathrm{~cm}^{-1}$ resolution, typically averaging 500 scans. Near- $\mathbb{R}$ spectra, typically 200 scans, were taken from 10,000 to $3,000 \mathrm{~cm}^{-1}$ at $4.0 \mathrm{~cm}^{-1}$ resolution. Dopant induced IR absorptions at 4,000 to $3,000 \mathrm{~cm}^{-1}, 1405$ to $1390 \mathrm{~cm}^{-1}, 1290 \mathrm{~cm}^{-1}$ and $800 \mathrm{~cm}^{-1}$ developed on warming. These absorptions began to appear between 125 and $140 \mathrm{~K}$ for $\mathrm{I}_{2}, 90$ and $130 \mathrm{~K}$ for $\mathrm{Br}_{2}$, near 60 $\mathrm{K}$ for $\mathrm{HCl}$, and near $50 \mathrm{~K}$ for $\mathrm{HBr}$. The same results were obtained with Shirakawa and Baker polyacetylene, suggesting that there was not a problem with thermal contact between the polymer sample and the substrate.

The temperature at which the prospective dopants begin to diffuse into the sample should be directly related to their melting points. The melting points of $\mathrm{I}_{2}, \mathrm{Br}, \mathrm{HCl}$, and $\mathrm{HBr}$ are $254 \mathrm{~K}, 160 \mathrm{~K}, 158 \mathrm{~K}$, and $185 \mathrm{~K}$, respectively. The temperatures at which these molecules begin to dope the polymer appear to be unrelated to their melting points; thus, the thermal barrier to doping must be due to something other than dopant diffusion.

\section{D.3 Conductivity Measurements}

A four-point probe was required to measure the resistance of doped samples. The ends of four 0.01 " platinum wires were pressed against the film in a collinear arrangement. A fixed current was passed between the end wires and the resistance was derived from the voltage difference between the central contacts. The contact resistances, which might have been large enough to mask the resistance being measured, were eliminated in this way. The measurement was made with a Keithley 197 digital multimeter. This experiment was done in parallel with the IR thernal doping experiment to show that IR activity and conductivity appear in the same temperature ranges. No change in resistivity was observed until the samples were warmed above $200 \mathrm{~K}$. Visual inspection found that the wires shadowed the sample, preventing exposure to dopants in the area where resistivity was measured. Good pressure contacts could not be maintained in probe geometries which might have eliminated the shadowing. The fact that the dopant did not reach the polymer 
shadowed by the wires, even at a temperature above that required for thermal doping, confirms the conclusion that diffusion is not the limiting step in low temperature doping.

\section{D.4 Photoassisted Doping at Low Temperatures}

Transient photoinduced IR active defects, with lifetimes on the millisecond time scale, have been observed during photoexcitation above the band gap. ${ }^{6}$ These $\mathbb{I R}$ absorptions have been assigned to charged defects, similar to those which develop on chemical doping. The electron energy levels in these charged defects are significantly different from those in the ground state chain. If the energy of electrons in the polymer made a major contribution to the thermal barrier for the doping reaction photoexcitation of the polymer could be expected to change the barrier.

While warming, polyacetylene was photolyzed with various frequencies of light, in the presence of $\mathrm{HCl}$ and $\mathrm{HBr}$, to determine the frequency dependence of the reaction temperature. Baker polyacetylene was used to assure good thermal contact with the substrate window. A multi-line $\mathrm{Ar}^{+}$ion laser, a Het.e laser, a Generai Electric AH4 medium pressure $\mathrm{Hg}$ lamp unfiltered and with either a germanium ( $\mathrm{v}<5,500 \mathrm{~cm}^{-1}$ ), silicon $\left(v<7,300 \mathrm{~cm}^{-1}\right)$, or Coming Glass 2-60 $\left(v<16,50^{\bigcap} \mathrm{cm}^{-1}\right)$ filter were used as photolysis sources. Of these photolysis sources, only the unfilterad $\mathrm{Hg}$ lamp was capable of exciting $\mathrm{HCl}$ or $\mathrm{HBr}$. No change in the temperature required for doping was observed in any of these photolysis ranges, for either $\mathrm{HCl}$ or $\mathrm{HBr}$.

The only changes observed in the IR spectra of polyacetylene photolysed in the presence of potential dopants, below the temperature at which the doping reaction occurs thermally without photolysis, were seen when the $\mathbb{R}$ probe beam was filtered with a germanium filter or a cut-on filter which blocked all light above $2,400 \mathrm{~cm}^{-1}$. With the probe beam filtered, there was an increase in absorbance of less than one one-hundredth of an absorbance unit at $1370 \mathrm{~cm}^{-1}$ when the sample was photolysed with $\mathrm{Si}(U<7,300$ $\mathrm{cm}^{-1}$ )-filtered or higher energy light. This absorption was lost on photolysis with $\mathrm{Ge}$ ( $\mathrm{v}<$ $5,500 \mathrm{~cm}^{-1}$ )-filtered light. Both the appearance and destruction of this absorption could be observed on the photolysis of polyacetylene even in the absence of dopant candidates.

\section{E Long-Lived Photoinduced IR Activity}

The effect of photoexcitation in the band gap on the IR spectra of undoped polyacetylene was investigated. The samples, Baker polyacetylene films dried onto CsI and Shirakawa polyacetylene films sandwiched between two substrate windows, were cooled in a displex mounted in an IBM IR/44 IR spectrometer. For these studies the samples were protected from external sources of light. A black flannel cloth was used to 
block room light. The $\mathbf{R}$ probe beam was filtered with an Optical Coatings Laboratory cuton filter which blocked all light above $v=2,400 \mathrm{~cm}^{-1}$. Spectra were taken from 400 to $2,300 \mathrm{~cm}^{-1}$ at $2 \mathrm{~cm}^{-1}$ resolution, averaging 200 scans. These spectra took less than 2 minutes to record; times listed for spectra are when the scans were begun.

The difference of the spectra taken of a sample of undoped polyacetylene cooled to $10 \mathrm{~K}$ in the dark and the same sample after photolysis with germanium filtered light shows a slighi: decrease in the absorbance at 1368,1289 and $600 \mathrm{~cm}^{-1}$. A more significant change, an increase in these $\mathbf{I R}$ absorption intensities, was observed when either as cooled or $\mathrm{Ge}\left(v<5,500 \mathrm{~cm}^{-1}\right)$-photolysed polyacetylene was photolysed with $\mathrm{CG}+\mathrm{H}_{2} \mathrm{O}(7,600$ $16,500 \mathrm{~cm}^{-1}$ )-filtered light. Various frequency ranges were tried: the maximum depletion of $\mathbb{R}$ activity was obtained with $\mathrm{Ge}\left(\mathrm{v}<5,500 \mathrm{~cm}^{-1}\right)$-filtered light, the maximum increase was obtained with $\mathrm{CG}+\mathrm{H}_{2} \mathrm{O}\left(7,600-16,500 \mathrm{~cm}^{-1}\right)$-filtered light. These changes were completely reversible, the $\mathbb{R}$ activity could be cycled between its maximum and minimum for days without any change in the range of intensity. Less than $20 \%$ of the possible $\mathbb{R}$ activity was observed in as cooled polyacetylene. When the flannel cloth was removed, the stray room light admitted was insufficient to have a significant effect on the change in IR activity pproduced by photolysis. On the other hand, no change in $\mathbf{I R}$ activity was observed after photolysis with $\mathrm{Ge}\left(v<5,500 \mathrm{~cm}^{-1}\right)$ - and $\mathrm{CG}+\mathrm{H}_{2} \mathrm{O}(7,600-16,500$ $\mathrm{cm}^{-1}$ )-filtered light when the $2,400 \mathrm{~cm}^{-1}$ cut-on filter in the probe beam was removed. This suggests that the flux from the probe beam is sufficient to establish an IR activity level characteristic of photolysis with the unfiltered probe beam by the time the specrta were recorded.

\section{E.1 Isotope Shift}

The difference spectra in Figure III-2 show the absorptions induced in polyacetylene and deuterated polyacetylene prepared by $\mathrm{Ge}\left(v<5,500 \mathrm{~cm}^{-1}\right)$-photolysis when they were photolysed with $\mathrm{CG}+\mathrm{H}_{2} \mathrm{O}\left(7,600-16,500 \mathrm{~cm}^{-1}\right)$-filtered light. The frequencies and full width at half maximum for these absorptions are given in Table III-3. The isotopic shift for the low frequency absorption should not be given too much attention, since the breadth of the absorption and poor signal to noise ratio do not allow for accurate frequency determination. In the higher frequency range two absorption peaks are replaced by only one in the spectrum of deuterated polyacetylene. The frequency shift from the more intense absorption in the undeuterated sample to the one absorption in the deuterated sample is 1.30 , indicating that the vibration involves a $\mathrm{C}-\mathrm{H}$ motion. 


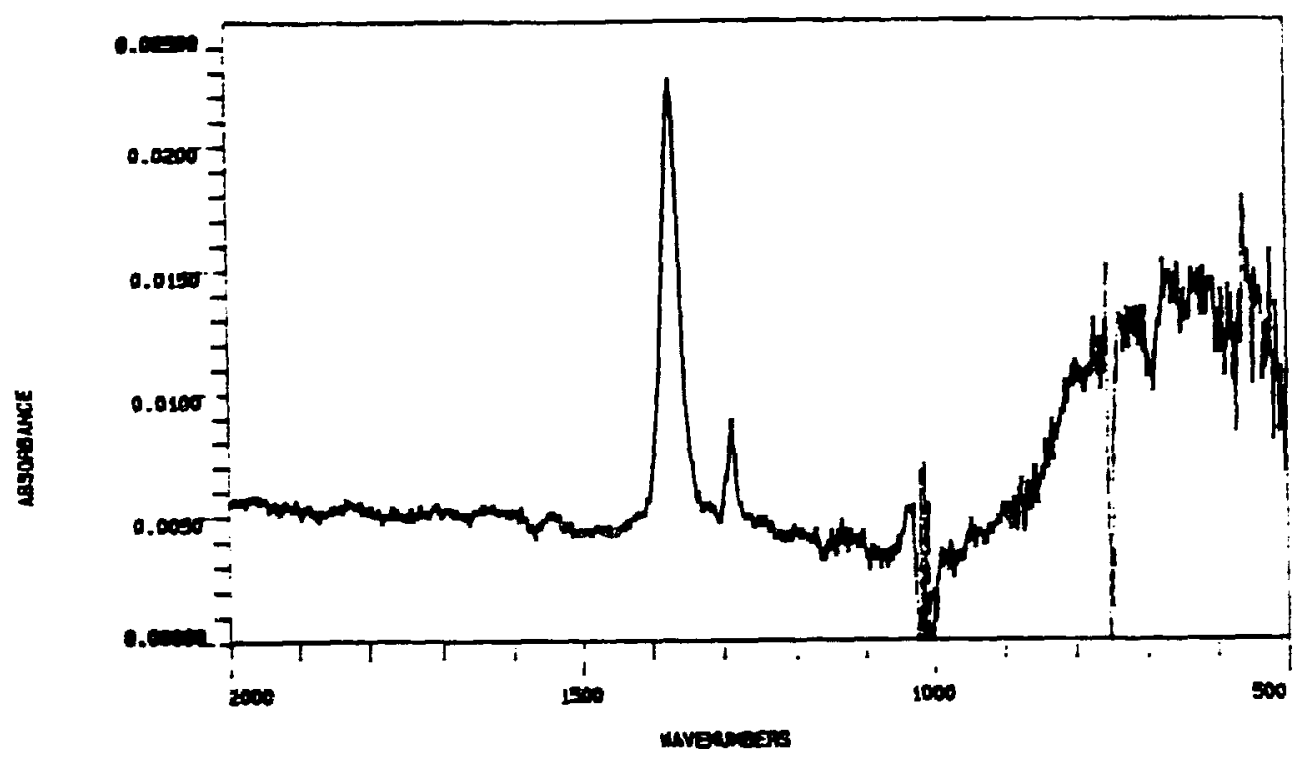

(A)

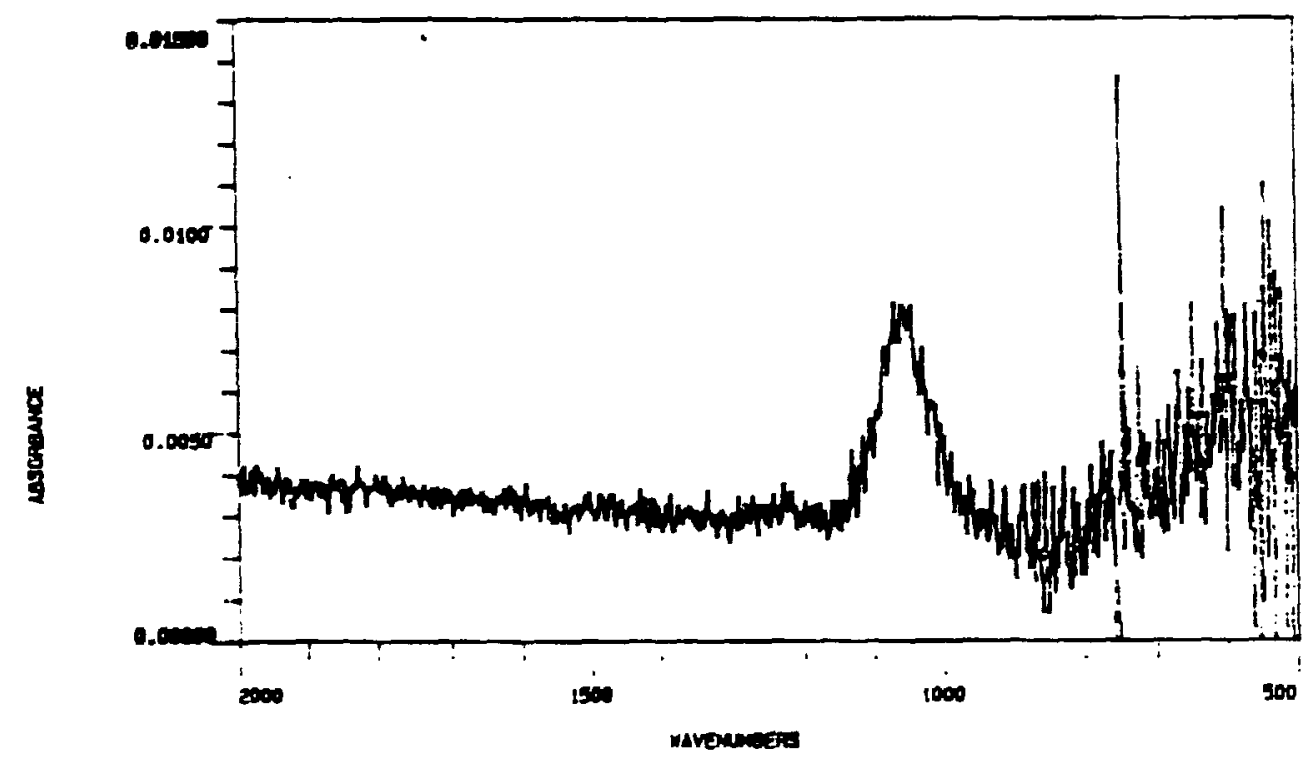

(B)

\section{Figure III-2 Photoinduced IR Absorptions}

Difference spectra showing the IR absorptions caused by photolysis with 7,600 - 16,500 $\mathrm{Cm}^{-1}$ light referenced to the same sample after phorolysis with $v<5,500 \mathrm{~cm}^{-1}$
A) $(\mathrm{CH})_{x}$
B) $(\mathrm{CD})_{\mathbf{x}}$ 
Table III-3 Persistent Photoinduced Absorptions

$$
\left(\mathrm{cm}^{-1}\right)^{(\mathrm{CH})_{\mathrm{X}}} \text { FWHM } \quad\left(\mathrm{cm}^{-1}\right)^{(\mathrm{CD})_{\mathrm{X}}} \text { FWHM } \quad \begin{gathered}
\text { isotope } \\
\text { shift }
\end{gathered}
$$

\begin{tabular}{rrrrr}
\hline 1368 & 27 & 1053 & 87 & 1.30 \\
1289 & 19 & & & \\
640 & -400 & 600 & -400 & 1.07 \\
\hline
\end{tabular}

Dependence on Isomer Content (Samule \#4) of Maximum Signal Obtained

\begin{tabular}{ccc} 
trans fraction & ABS units & ABS/trans fraction \\
\hline 0.09 & 0.005 & 0.056 \\
0.47 & 0.013 & 0.28 \\
0.64 & 0.018 & 0.28 \\
\hline
\end{tabular}

Temperature Dependence (Sample \#2) of Maximum Signal Obtained

\begin{tabular}{ccccccc} 
Temperature & $10 \mathrm{~K}$ & $25 \mathrm{~K}$ & $50 \mathrm{~K}$ & $75 \mathrm{~K}$ & $100 \mathrm{~K}$ & $125 \mathrm{~K}$ \\
\hline $\begin{array}{c}\text { Maximum } \\
\begin{array}{c}\text { Signal } \\
\text { (ABS units) }\end{array}\end{array}$ & 0.0128 & 0.0110 & 0.0101 & 0.0072 & 0.0050 & 0.0025
\end{tabular}




\section{E.2.a Dependence of the Signal Intensity On Isomer Content}

The maximum signal obtainable is given as the measured peak height of the 1369 $\mathrm{cm}^{-1}$ peak in absorbance units, observed in the difference spectra between the sample photolyzed with $\mathrm{Ge}\left(v<5,500 \mathrm{~cm}^{-1}\right)$ - and $\mathrm{CG}+\mathrm{H}_{2} \mathrm{O}\left(7,600-16,500 \mathrm{~cm}^{-1}\right)$-filtered light. The maximum signal obtainable was determinea for the same sample at three different isomeric ratios (Table III-3). To do this the sample was removed from the Displex, thermally annealed and returned to the Displex, twice. The maximum signal obtainable increases with increasing trans content. It has been shown by other workers ${ }^{7}$ that the $\log$ of the concentration of neutral defects increases linearly with trans content. It is not clear from this data whether the maximum signal size is dependent on the number of trans units or neutral defects. Baker polyacetylene, reported to have ten times fewer neutral defects than Shirakawa polyacetylene, produces less than one tenth the IR activity per trans unit, as determined by $\mathbb{R}$ absorption at $1015 \mathrm{~cm}^{-1}$. This suggests that the induced $\mathbb{R}$ activity depends on the presence of neutral defects.

\section{E.2.b Temperature Dependence of Signal Intensity}

The maximum obtainable signals, measured for the same sample at several temper:tures, are also given in Table III-3. The signal decreases linearly with increasing temperature from 10 to $125 \mathrm{~K}$. Above $125 \mathrm{~K}$ the signal intensity is too small to report with any accuracy. The intensity of transient above-gap photoinduced IR absorptions also decreases linearly with increasing temperature below $150 \mathrm{~K}$, and above this temperature it drops off sharply. 8

\section{E.3 Dark Decay}

Previously, IR absorptions induced by photolysis above the band gap have been observed to be transient with a lifetime on the millisecond timescale. ${ }^{6}$ The IR abso-ptions observed to develop on photolysis with $\mathrm{CG}+\mathrm{H}_{2} \mathrm{O}\left(7,600-16,500 \mathrm{~cm}^{-1}\right)$-filtered light, in this study, persisted for a much longer time. These absorptions decay slowly in the absence of light. The decay of the photoinduced IR absorption at $1369 \mathrm{~cm}^{-1}$ in the dark is shown in Figure III-3. This decay cannot be fit to an exponential or biexponential rate. Fast at first, decreasing with signal size, the decay rate appears to be a superpositon of many exponentiais.

The reason for this dec. 2 , has not been experimentally determined. The effect of the probe beam on the decay kinetics was tested. The decay of the signal intensity with the sample left in the beam and with the sample rotated out of the beam path betweer. spectra are given in Table III-4. The piobe beam had no discernible effect on the rate of decay. 

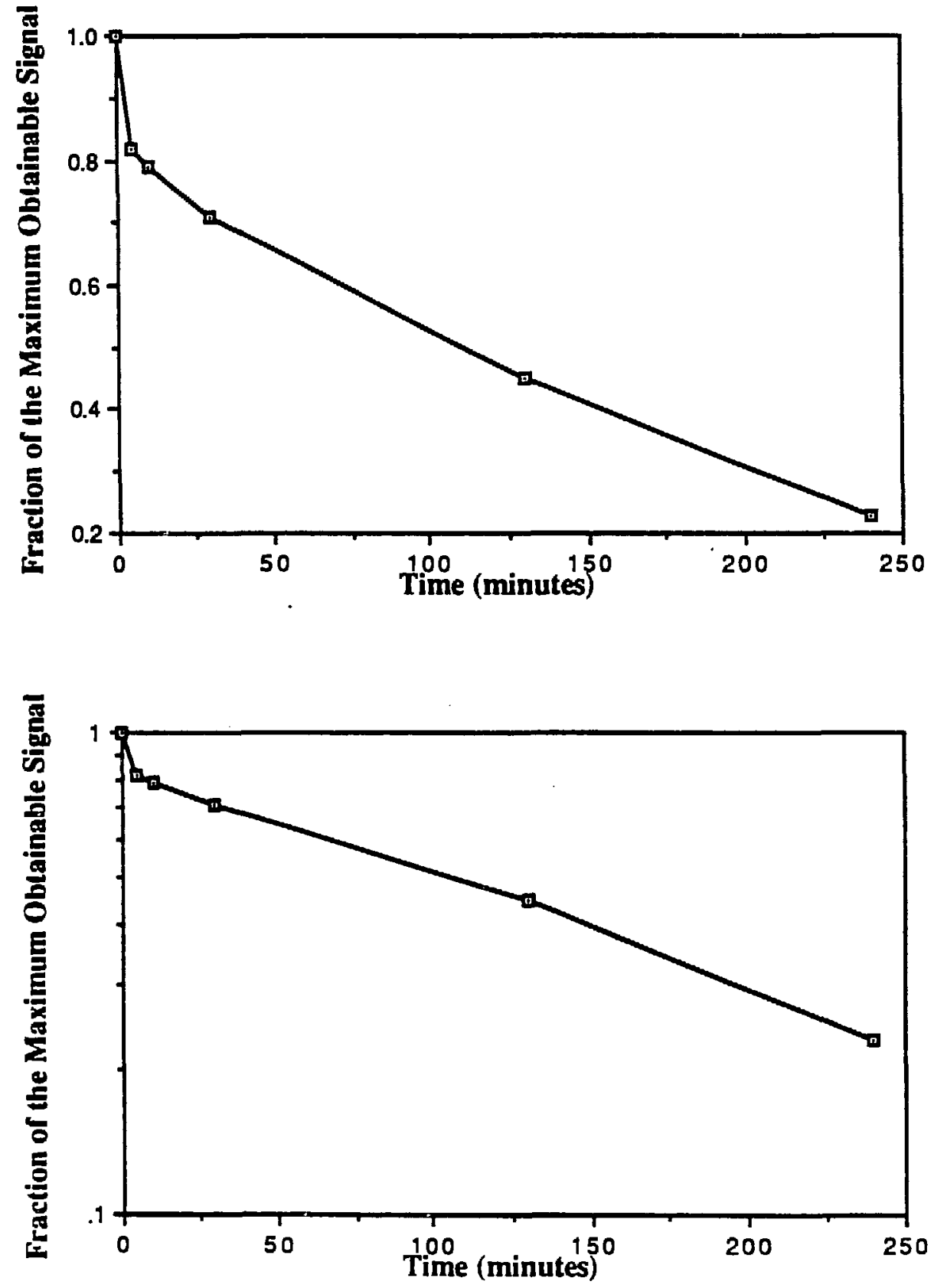

Figure III-3 Decay of $\mathbb{R}$ Activity in the Dark 
Table III-4 Signal Decay in the Dark (fraction of the maximum obtainable signal)

Effect of Background Iradiation (Sample $\$ 2$ at $10 \mathrm{~K}$ )

Time Since

Photolysis

(mins)

$$
\text { CsI IR CsI dark } \quad \mathrm{CaF}_{2} \mathrm{IR} \quad \mathrm{CaF}_{2} \text { dark }
$$

\begin{tabular}{rllll}
\hline 0 & 1.00 & 1.00 & 1.00 & 1.00 \\
5 & 0.81 & 0.84 & 0.81 & 0.89 \\
10 & 0.75 & 0.79 & 0.74 & 0.79 \\
30 & 0.67 & 0.71 & 0.65 & 0.68 \\
130 & 0.53 & 0.45 & 0.51 & 0.53 \\
\hline
\end{tabular}

Isomer and Isotope Dependence of Signal Decay in the Dark (10 K)

\begin{tabular}{cccc}
$\begin{array}{c}\text { Time Since } \\
\text { Photolysis } \\
\text { (mins) }\end{array}$ & $\begin{array}{c}\text { Sample \#1 } \\
60 \% \text { trans } \\
\text { (CD) }\end{array}$ & $\begin{array}{c}\text { Sample \#2 } \\
70 \% \text { trans } \\
\text { (CH) }\end{array}$ & $\begin{array}{c}\text { Sample \#3 } \\
85 \% \text { trans } \\
\text { (CD) }\end{array}$ \\
\hline 0 & 1.00 & 1.00 & 1.00 \\
5 & 0.92 & 0.82 & 0.78 \\
10 & 0.92 & 0.79 & 0.65 \\
30 & 0.83 & 0.71 & 0.46 \\
130 & 0.65 & 0.45 & - \\
240 & - & 0.23 & - \\
330 & 0.54 & - & - \\
\hline
\end{tabular}

Temperature Dependence of Signal Decay in the Dark (Sample \#2)

Time Since

Photolysis (mins)

$10 \mathrm{~K} \quad 25 \mathrm{~K} \quad \begin{gathered}\text { Temperature } \\ 50 \mathrm{~K}\end{gathered}$

$75 \mathrm{~K}$

$125 \mathrm{~K}$

$\begin{array}{rlllll}0 & 1.00 & 1.00 & 1.00 & 1.00 & 1.00 \\ 5 & 0.82 & 0.79 & 0.82 & 0.81 & 0.70 \\ 10 & 0.79 & 0.74 & 0.73 & 0.76 & 0.71 \\ 30 & 0.71 & 0.65 & 0.58 & 0.69 & 0.48 \\ 130 & 0.45 & 0.45 & 0.42 & & \end{array}$


It was suspected that photolysis by black body radiation from the room temperature surroundings might be the cause of the dark decay. The observed dark decay rate could be obtained by photolysis at $4,000 \mathrm{~cm}^{-1}$ with $2.8 \times 10^{20}$ photons per second. The number of black body photons, $v<5,500 \mathrm{~cm}^{-1}$, incident on the sample were calculated to be fewer than $1.7 \times 10^{16}$ per second, more than a million times fewer than would be necessary to account for the decay.

The low frequency photoinduced absorption is believed to be associated with movement of charged defects along the polymer chain. The possibility that excitation of this motion is responsible for the dark decay was also eliminated. $\mathrm{CaF}_{2}$ substrate windows were used to sandwich the sample. Once cooled the $\mathrm{CaF}_{2}$ acted as a cold filter protecting the sample from all irradiation below $1,000 \mathrm{~cm}^{-1}$. The dark decay rate observed with the cold filter was the same as that for the same sample between CsI substrate windows (Table III-4).

\section{E.3.a Dependence of the Dark Decay Rate on Isomer Content}

The kineric data for the decay of the photoinduced IR activity in the dark are given in Table III-4. The signal intensities are reported as the fraction of the maximum obtainable peak height for the absorption at $1369 \mathrm{~cm}^{-1}$. The isotopic substitution does not appear to have enough of an effect on the rate of dark decay to disrupt the trend observed for the effect of isomer content. The rate at which the photoinduced IR activity decayed in the dark increased with trans content.

No dark decay was observed in the photoinduced IR absorptions in Baker polyacetylene. Baker polyacetylene, purified in a Soxhlet extractor is typically $98 \%$ trans. The signal observed in Baker polyacetylene is less than one tenth that which would be seen in a Shirakawa sample with the same absorption at $1015 \mathrm{~cm}^{-1}$, the trans C-H out-of-plane bend. It is possible that the fast components of the dark decay in these samples are so fast that only the slow part remained when the the signal was observed. This possible explanation for the smaller signal in Baker polyacetylene weakens the argument that the signal size is dependent on neutral defect concentration.

\section{E.3.b Temperature Dependence of the Dark Decay Rate}

The dark decay kinetics were recorded for the same sample at several temperatures (Table III-4). The maximum obtainable signal decreases with temperature, but the percentage of the signal that decays in a given time is not effected by temperature. 


\section{E.4 Frequency Dependence}

The kinetics for the growth or loss of IR activity were determined for narrow frequency ranges between 2,800 and $11,000 \mathrm{~cm}^{-1}$. The intensities for the growth kinetics are reported as the fraction of the maximum obtainable signal induced in a sample, whose IR activity had been minimized with $\mathrm{Ge}\left(v<5,500 \mathrm{~cm}^{-1}\right)$-filtered light, by photolysis with a specified number of photons in the frequency range of interest. Similarly samples were prepared for depletion kinetics by maximizing their signal with $\mathrm{CG}+\mathrm{H}_{2} \mathrm{O}(7,600-16,500$ $\mathrm{cm}^{-1}$ )-filtered light. The data for the depletion and growth of IR absorption in sample \#2 are given in Tables III-5 and III-6. Some of this data is plotted if Figures III-4 and III-5. Sample \# 2 had the best near-IR transmission of any sample of similar thickness, providing a relatively large photoinduced signal with a wide region of low optical density.

\section{E.5 Depletion kinteics}

The rate of decay during photolysis at $2,800 \mathrm{~cm}^{-1}$ is the same as in the dark. The signal is depleted more rapidly during photolysis at frequencies between $3,000 \mathrm{~cm}^{-1}$ and $6,950 \mathrm{~cm}^{-1}$ than in the dark. The relative rates of depletion at different frequencies should be directly related to the absorption coefficient of the state through which depletion proceeds. The multi-exponential nature of the rate and superposition of the dark decay make quantitative data analysis impossible.

As with the dark decay, the groweh and depletion rates were not properly fit by an exponential curve. The data sets are small enough that they were easily fit to a biexponential decay curve with all five parameters changing with frequency (Table III-7). Reasonable fits could not be obtained at all frequencies if either the rates or populations were fixed. This suggests that there are many different sites with slightly different frequency dependent depletion rates. Thus, the biexponential model is not detailed enough for the system; however, a more involved model could not be used because the data sets were too small to be fit with any more parameters.

The five parameter fit, Signal $(t)=A+B e^{-C t}+D e^{-E t}$, divides the signal into three population groups: (B) those which are subject to rapid depletion with an average relative rate constant (C), (D) those which are depleted at intermediate rates, with an average relative rate constant $(E)$, and $(A)$ those whose depletion rate is extremely slow Figure III6 shows the distribution of these populations as a function of frequency. The average fast and intermediate rates for each frequency are shown in Figure III-7. Abrupt deviations in the values of these parameters as a function of frequency can be considered artifacts of small data sets. When viewed together these parameters do indicate the relative depletion efficiency of the various photolysis frequencies. 
Table III-5 Depletion Kinetics (fraction of the maximum obtainable signal)

\begin{tabular}{llllllllll} 
Photolysis & \multicolumn{1}{c}{ Number of Photons $\left(\times 10^{23}\right)$} \\
Frequency & 0 & 5 & 15 & 50 & 100 & 500 & 1000 & 5000 & S.S.
\end{tabular}

\begin{tabular}{|c|c|c|c|c|c|c|c|c|c|}
\hline 2,800 & 1.00 & 0.89 & 0.85 & 0.79 & 0.75 & 0.61 & 0.59 & & \\
\hline 3,000 & 1.00 & 0.85 & 0.81 & 0.74 & 0.66 & 0.54 & 0.46 & & \\
\hline 3,200 & 1.00 & 0.79 & 0.70 & 0.61 & 0.60 & 0.52 & 0.43 & & \\
\hline 3,400 & 1.00 & 0.82 & 0.67 & 0.57 & 0.56 & 0.39 & 0.38 & & \\
\hline 3,600 & 1.00 & 0.78 & 0.65 & 0.53 & 0.46 & 0.32 & 0.29 & & \\
\hline 3,800 & 1.00 & 0.67 & 0.58 & 0.40 & 0.33 & 0.27 & 0.25 & & \\
\hline 4,000 & 1.00 & 0.69 & 0.53 & 0.40 & 0.32 & 0.20 & 0.16 & & \\
\hline 4,200 & 1.00 & 0.63 & 0.54 & 0.38 & 0.27 & 0.16 & 0.13 & & \\
\hline 4,400 & 1.00 & 0.66 & 0.53 & 0.33 & 0.26 & 0.13 & 0.13 & & \\
\hline 4,600 & 1.00 & 0.66 & 0.52 & 0.34 & 0.25 & 0.13 & 0.09 & & \\
\hline 4,800 & 1.00 & 0.66 & 0.48 & 0.36 & 0.25 & 0.13 & 0.10 & & \\
\hline 5,000 & 1.00 . & 0.73 & 0.53 & 0.39 & 0.29 & 0.15 & 0.15 & & \\
\hline 5,200 & 1.00 & & 0.58 & 0.39 & 0.31 & 0.13 & & & \\
\hline "5,810" & 1.00 & 0.82 & 0.62 & 0.54 & 0.47 & 0.31 & 0.29 & 0.25 & 0.25 \\
\hline "6,950" & 1.00 & & 0.83 & 0.68 & & 0.52 & & 0.47 & 0.47 \\
\hline$" 8,690^{n}$ & 1.00 & & & 0.92 & & & 0.93 & & 0.93 \\
\hline "9,300" & 1.00 & & 1.00 & 1.00 & & 1.00 & & & 1.00 \\
\hline "10,700" & 1.00 & & & 0.89 & & 0.85 & 0.85 & 0.84 & \\
\hline
\end{tabular}


Table III-6 Growth Kinetics (fraction of the maximum obtainable signal)

\begin{tabular}{|c|c|c|c|c|c|c|c|c|c|}
\hline \multirow{2}{*}{$\begin{array}{l}\text { Photolysis } \\
\text { Frequency }\end{array}$} & \multicolumn{9}{|c|}{ Number of Photons $\left(\times 10^{23}\right)$} \\
\hline & 0 & 5 & 10 & 15 & 50 & 100 & 500 & 5000 & S.S. \\
\hline 4,800 & 0.00 & & & & 0.02 & & 0.00 & & \\
\hline 5,000 & 0.00 & & & & & & & & \\
\hline 5,200 & 0.00 & & & 0.07 & & & 0.06 & & \\
\hline${ }^{-5,810^{\prime}}$ & 0.00 & & & & 0.12 & & 0.25 & 0.25 & 0.25 \\
\hline$" 6,950 "$ & 0.00 & & 0.17 & & 0.32 & & 0.47 & 0.46 & 0.46 \\
\hline$" 8,690 "$ & 0.00 & 0.50 & 0.70 & 0.756 & 0.88 & 0.95 & & & 0.94 \\
\hline${ }^{-9}, 300^{n}$ & 0.00 & 0.48 & 0.66 & 0.72 & 0.93 & 0.96 & 1.00 & & 1.00 \\
\hline$" 10,700^{n}$ & 0.00 & 0.25 & 0.39 & 0.47 & 0.69 & 0.78 & 0.82 & 0.82 & \\
\hline
\end{tabular}




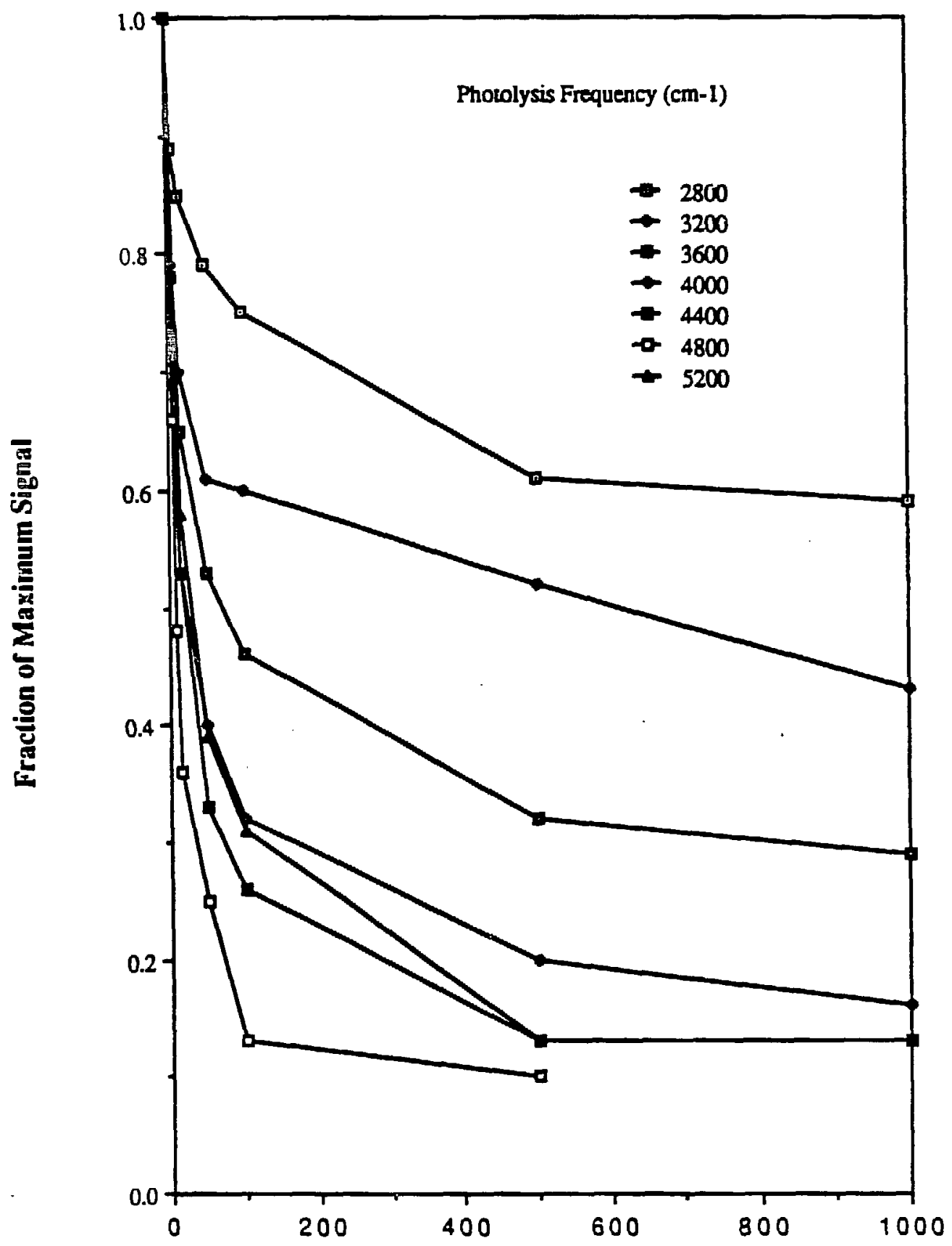

Number of Photons (x10E23) 


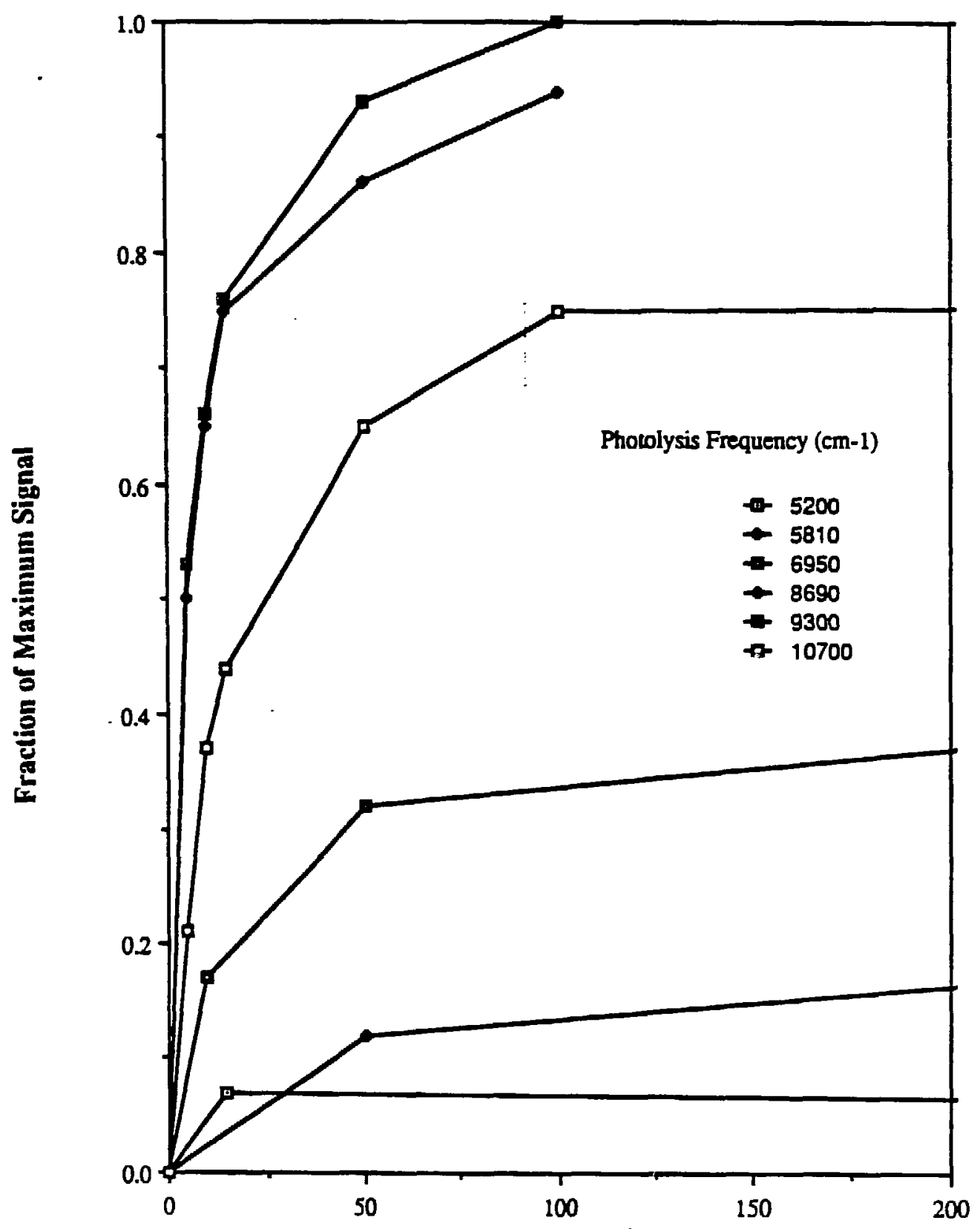

Number of Photons (x10E23)

Figure III-5 Growth of Photoinduced IR Activity 
Table III-7 Five Parameter Fit for Depletion Kinetics

$$
\operatorname{Signal}(t)=A+B e^{-C t}+D e^{-E t}
$$

\begin{tabular}{llllll} 
Frequency $\left(\mathrm{cm}^{-1}\right)$ & A & B & C & D & E \\
\hline 2,800 & 0.59 & 0.14 & 0.28 & 0.27 & 0.0056 \\
3,000 & 0.48 & 0.18 & 0.30 & 0.34 & 0.0054 \\
3,200 & 0.20 & 0.36 & 0.14 & 0.44 & 0.0006 \\
3,400 & 0.37 & 0.37 & 0.13 & 0.26 & 0.0042 \\
3,600 & 0.30 & 0.34 & 0.18 & 0.36 & 0.083 \\
3,800 & 0.26 & 0.31 & 0.54 & 0.43 & 0.021 \\
4,000 & 0.18 & 0.44 & 0.21 & 0.38 & 0.010 \\
4,200 & 0.15 & 0.37 & 0.48 & 0.48 & 0.014 \\
4,400 & 0.13 & 0.39 & 0.31 & 0.48 & 0.015 \\
4,600 & 0.11 & 0.41 & 0.28 & 0.48 & 0.013 \\
4,800 & 0.12 & 0.47 & 0.23 & 0.41 & 0.011 \\
5,000 & 0.15 & 0.45 & 0.23 & 0.42 & 0.010 \\
5,200 & 0.12 & 0.51 & 0.09 & 0.37 & 0.0065 \\
& & & & & \\
\hline
\end{tabular}




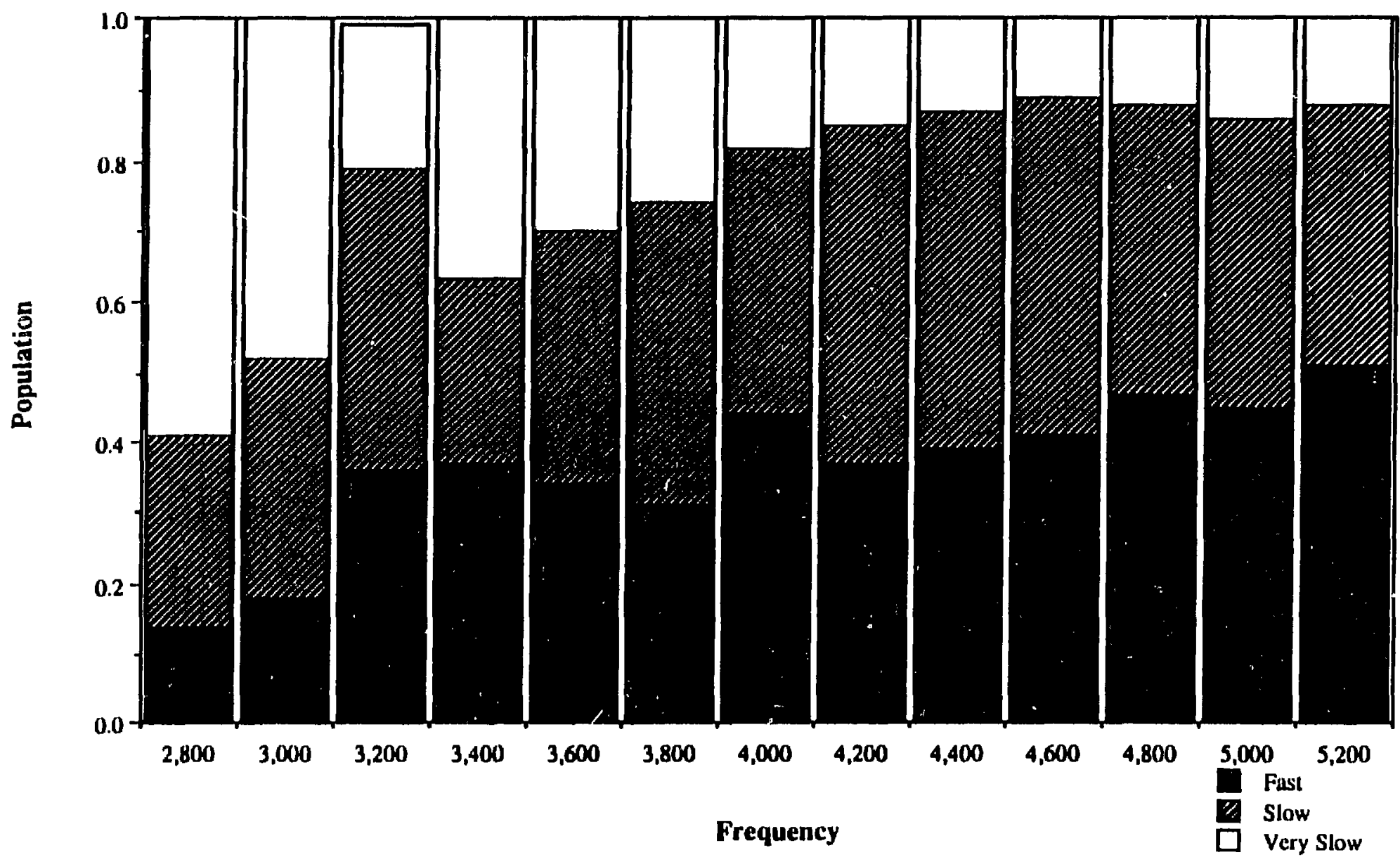

Figure III-6 Populations from the Five-Parameter Fit

Populations subject to very slow (A - white), intermediate (D - shaded), and rapid photodepletion (B - black) 


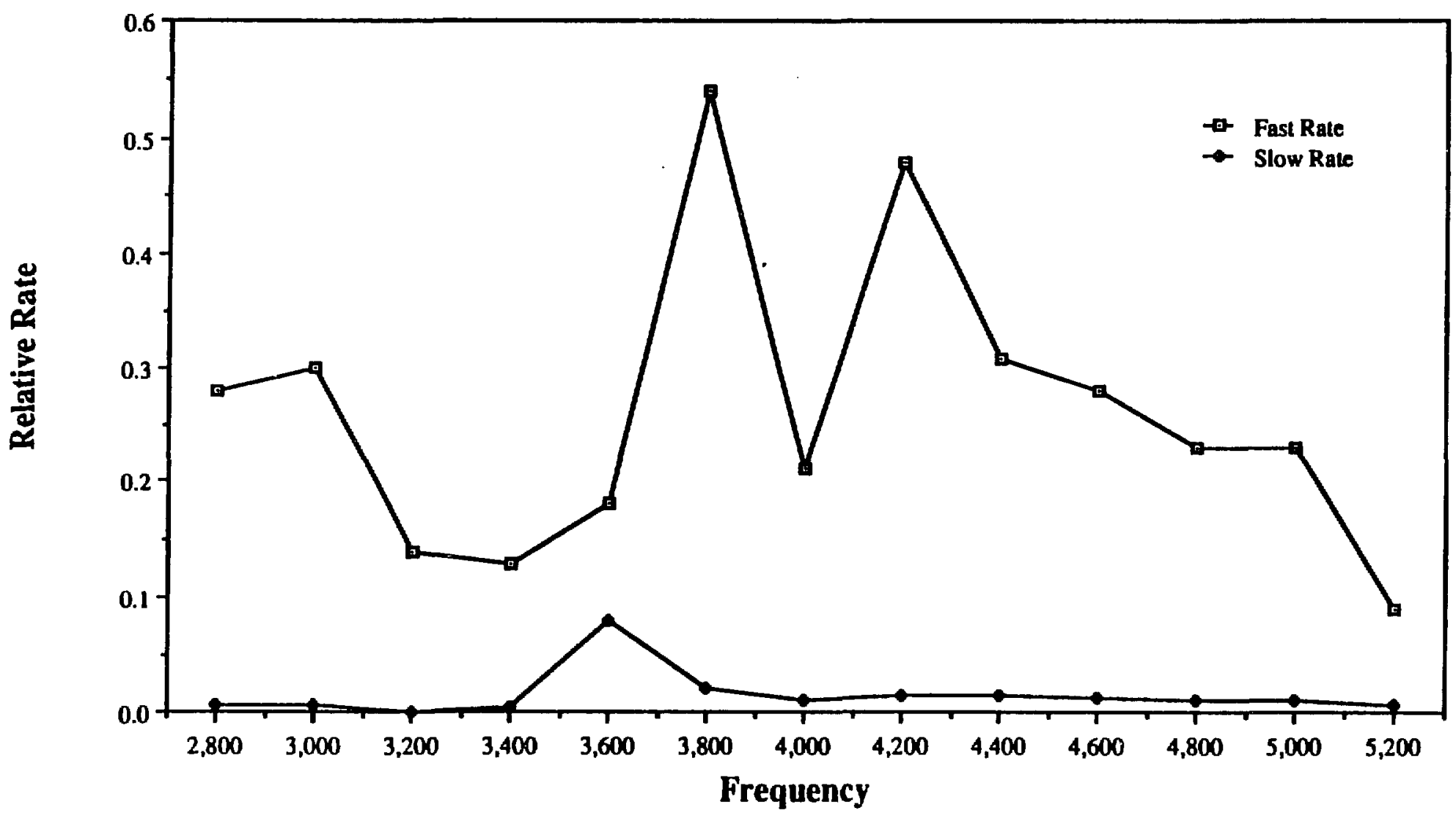

Figure III-7 Rates from the Five-Parameler Fit

Fast (upper) and intermediate (lower) rates for photodopletion

$\stackrel{\infty}{\sim}$ 
There is a significant increase in the population subject to discernible depletion at $3,200 \mathrm{~cm}^{-1}$. At $3,600 \mathrm{~cm}^{-1}$ the rate at which the intermediate population is depleted peaks. The average rate for fast depletion speeds up at $3,800 \mathrm{~cm}^{-1}$. The population distributions are fairiy constant above $3,600 \mathrm{~cm}^{-1}$. The depletion rates start to decrease above 4,200 $\mathrm{cm}^{-1}$. From this a broad distribution of transitions can be assumed. This distribution has an onset near $3,200 \mathrm{~cm}^{-1}$ and a peak near $4,000 \mathrm{~cm}^{-1}$.

\section{E.4.b Growth Kinetics}

The growth of the photoinduced $\mathbb{R}$ absorptions is fastest and most complete at frequencies near $9,300 \mathrm{~cm}^{-1}$. Photolysis of a $\mathrm{Ge}\left(v<5,500 \mathrm{~cm}^{-1}\right)$-photolysed sample with $10,300 \mathrm{~cm}^{-1}$ light failed to produce the maximum obtainable signal. It is believed that this is because production at this frequency is not fast enough to maintain the maximum signal while the signal is being depleted by the dark decay process not photodepletion. The rate at which $\mathbb{R}$ absorption is photoproduced at this frequency may be small because of low flux (Table III-1), poor sample transmission (Table III-2), or a small absorption cross section.

At long times, photolysis of either $\mathrm{Ge}\left(v<5,500 \mathrm{~cm}^{-1}\right)$ - or $\mathrm{CG}+\mathrm{H}_{2} \mathrm{O}(7,600$ $16,500 \mathrm{~cm}^{-1}$ )-photolysis prepared samples at frequencies between 4,800 and $8,690 \mathrm{~cm}-1$ results in an intermediate, steady state, IR signal intensity. The approach to this steady state value from the maximum signal by depletion with $5,810 \mathrm{~cm}^{-1}$ light and from the minimum signal photoinduced with $5,810 \mathrm{~cm}^{-1}$ light is shown in Figure III-8. This steady state value is determined by the relative efficiencies of the production and depletion processes at that frequency. The steady state values are reproducible to within $5 \%$ from sample to sample except for samples which are opaque in the photolysis region. The kinetics for photolysis above $5,000 \mathrm{~cm}^{-1}$ cannot be modeled in an appropriate manner because the samples are not optically thin in this frequency range, and the dark decay and multi-exponential problems still come into play.

It was assumed that there were a finite number of IR inactive sites, I, which could become IR active, $A$, on photolysis. The growth and depletion kinetic data for IR activity induced by photolysis frequencies between 5,810 and $10,700 \mathrm{~cm}^{-1}$ (from Tables $\mathrm{III}-5$ and III-6) was fit to the rate equation for the first order reversible photoreaction:

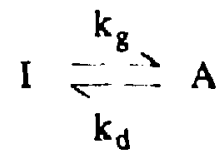




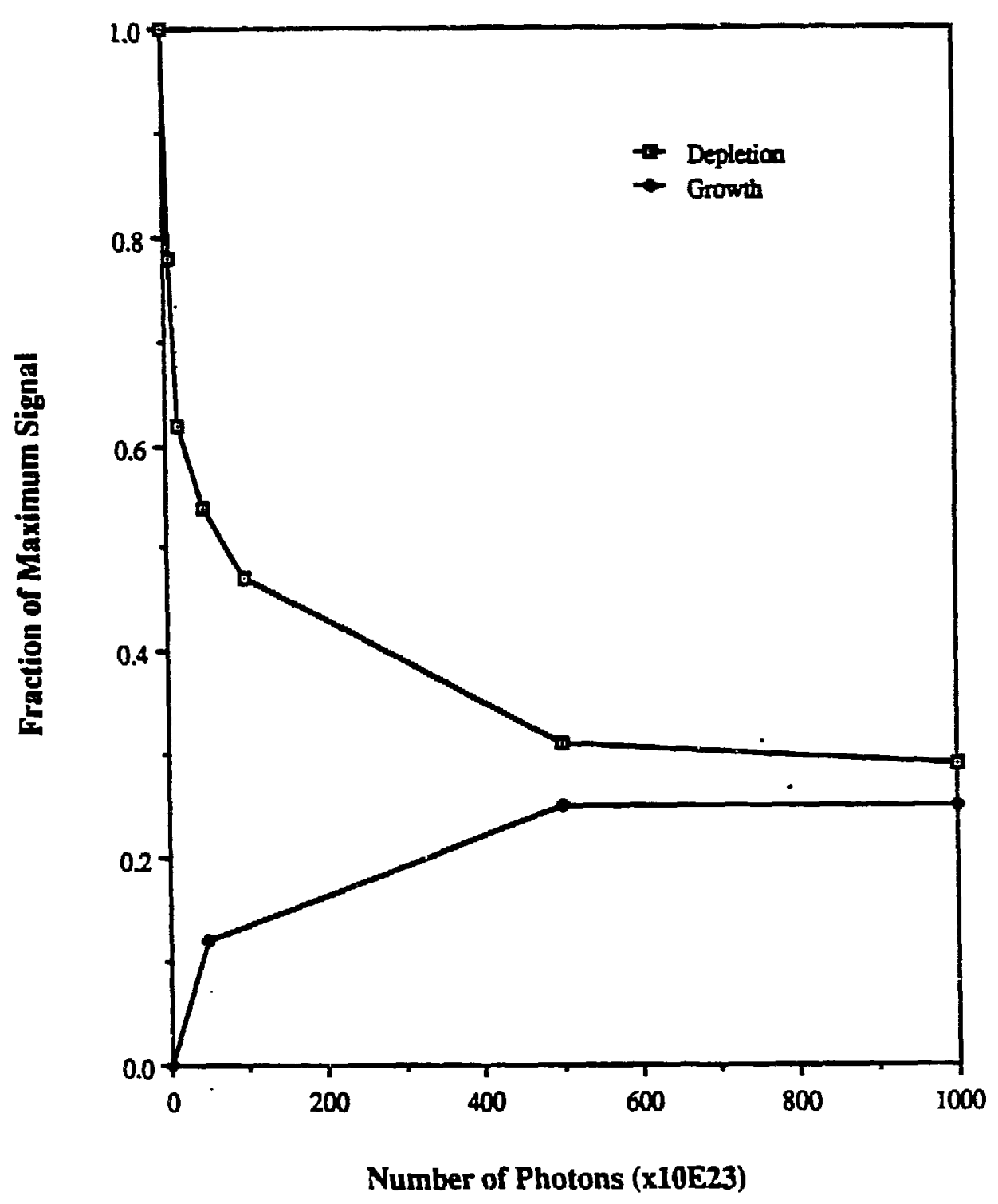

Figure III-8 Growth and Depletion of IR Activity with Photolysis at $5,810 \mathrm{~cm}^{-1}$

A) Photodepletion of the maximum obtainable photoinduced IR signa! by photolysis with $5,810 \mathrm{~cm}^{-1}$ light

B) Photoproduction of IR activity by photolysis with $5,810 \mathrm{~cm}^{-1}$ light, after first minimizing the $I R$ activity with $v<5,500 \mathrm{~cm}^{-1}$ phoiolysis. 
where $\mathrm{kg}_{\mathrm{g}}$ is the effective rate constant for the grcwth of IR activity, and $\mathrm{k}_{\mathrm{d}}$ is the effective rate constant for the depletion of $\mathbb{R}$ activity. Since a one to one correspondence between I and $A$ is assumed and $I$ and $A$ are defined as fractions (peak height divided by the maximum obtainable peak height for the $1369 \mathrm{~cm}^{-1}$ absorption):

$$
[\Pi=1-[A]
$$

Using this relationship, the rate of change in $[A]$ per photon, $p$ :

$$
d[A] / d p=k_{g}[M]-k_{d}[A]
$$

becomes:

$$
d[A] / d p=k_{g}-\left(k_{d}+k_{g}\right)[A]
$$

After long photolyses when steady state concentration is reached:

$$
d[A] / d p=0=k_{g}-\left(k_{d}+k_{g}\right)\left[A_{s s}\right]
$$

Thus:

$$
k_{g} /\left(i_{d}+k_{g}\right)=\left[A_{s s}\right]
$$

a new variable, $y$, is defined as:

$$
y=k_{g}-\left(k_{d}+k_{g}\right)[A \text {. }
$$

thus:

$$
d y / d p=-\left(k_{d}+k_{g}\right) d[A] / d p
$$

Substituting in equations (4) and (5), equation (2) becomes:

$$
-1 /\left(k_{d}+k_{g}\right) d y / d p=y
$$

This equation is integrated from $(, j$ to get: 


$$
\ln \left[\left(k_{g}-\left(k_{d}+k_{g}\right)[A]\right) /\left(k_{g}-\left(k_{d}+k_{g}\right)\left[A_{0}\right]\right)\right]=-\left(k_{g}+k_{d}\right) p
$$

Substituting in equation (3) equation (6) becomes:

$$
\ln \left[\left(A-A_{s s}\right) /\left(A_{0}-A_{s s}\right)\right]=-\left(k_{g}+k_{d}\right) p
$$

The sums of the effective rate constants for the growth and depletion kinetic data for photolysis frequencies between $5,810 \mathrm{~cm}^{-1}$ and $10,700 \mathrm{~cm}^{-1}$ were determined using equation (7). Only points where the change in IR absorption was $>5 \%$ (clearly above the noise) were considered. Figure III-9 shows the fit obtained with equation (7) for the growth kinetics during photolysis at $8,690 \mathrm{~cm}^{-1}$. The non-linearity of this plot, In [ (A $\left.\left.A_{s s}\right) /\left(A_{0}-A_{s s}\right)\right]$ vs, the number of photons $\left(x_{1023}\right)$, demonstrates that the growth kinetics, like the decay kinetics, are of a multi-exponential nature. The individual effective rate constants were determined from the slopes in equation (7), which give their sums, and the steady state signals, using equation (3). The effective rate constants and their sums are given in Table III-8. There is a clear increase in the effective rate constant up to 9,300 $\mathrm{cm}^{-1}$. This corresponds to an increase in the absorption coefficient of a transition which, when excited, induces IR activity. Since the sample transmittance decreases rapidly at higher frequencies, the fact that the effective rate constant at $10,700 \mathrm{~cm}^{-1}$ is smaller than that at $9,300 \mathrm{~cm}^{-1}$ does not guarantee that the extinction coefficient is smaller at 10,700 $\mathrm{cm}^{-1}$ than at $9,300 \mathrm{~cm}^{-1}$.

\section{E.5 Asymmetry}

Another indicator of the variation in response to different photolysis frequencies is the location of the $1369 \mathrm{~cm}^{-1}$ photoinduced IR absorption. Photolysis at low frequencies depletes this band asymmetrically. Figure III-10A shows the maximum obtainable signal at $1369 \mathrm{~cm}^{-1}$, induced by $\mathrm{CG}+\mathrm{H}_{2} \mathrm{O}\left(7,600-16,500 \mathrm{~cm}^{-1}\right)$-photolysis, referenced to a $\mathrm{Ge}(\mathrm{v}$ $<5,500 \mathrm{~cm}^{-1}$ )-photolysed sample. This signal, reduced to half-height by photolysis at $3,600 \mathrm{~cm}^{-1}$, is also shown referenced both to the $\mathrm{Ge}\left(v<5,500 \mathrm{~cm}^{-1}\right)$-photolysed sample (signal remaining), and to the $\mathrm{CG}+\mathrm{H}_{2} \mathrm{O}\left(7,600-16,500 \mathrm{~cm}^{-1}\right)$-photolysed sample (signai depleted). The locations of the remaining and depleted absorptions for different photolysis frequencies are reported in Table III-9. Depletion during photolysis at $2,800 \mathrm{~cm}^{-1}$, which is believed to be entirely due to dark decay, is symmetric. This is confusing since the dark decay normally depletes the $1369 \mathrm{~cm}^{-1}$ band asymmetrically, low frequency side first. Low energy photolysis light, 3,000 to $4,000 \mathrm{~cm}^{-1}$, depletes the low frequency side of the 


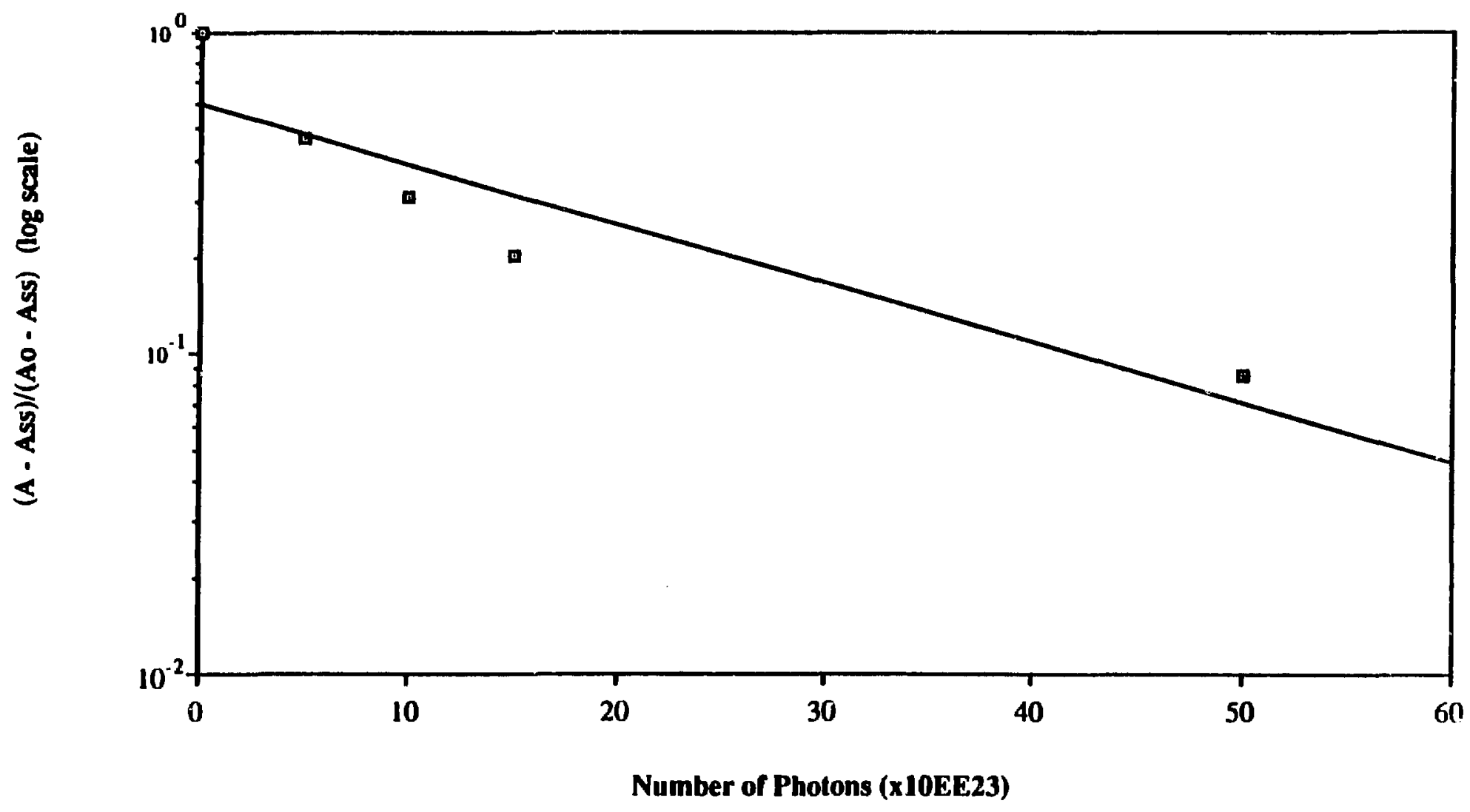

Figure III-9 Poor Fit

Poor fit obtained by fitting the growth kinetic data for photolysis at $8,690 \mathrm{~cm}^{-1}$ to a reversible first order rate equation 
Table III-8 Effective Rate Constants (change in fraction/photon x 1025)

\begin{tabular}{cccc}
$\begin{array}{c}\text { Frequency } \\
\left(\mathrm{cm}^{-1}\right)\end{array}$ & $\begin{array}{c}\text { Sum of } \\
\text { Rate Constants } \\
\text { From Growth }\end{array}$ & $\begin{array}{c}\text { Sum of } \\
\text { Rate Constants } \\
\text { From Depletion }\end{array}$ & $\begin{array}{c}\text { Average of } \\
\text { the Sums }\end{array}$ \\
\hline 5,810 & 0.57 & 0.7 & 0.64 \\
6,950 & 0.92 & 0.2 & 0.56 \\
8,690 & 1.85 & & 1.85 \\
9.300 & 2.05 & & 2.05 \\
10,700 & 0.85 & & 0.85 \\
\hline
\end{tabular}

Frequency

$\left(\mathrm{cm}^{-1}\right)$

5,810

6,950

8,690

9,300

10,700
Growth

Rate Constant
Depletion

Rate Constant

\begin{tabular}{lll}
\hline 5.810 & 0.16 & 0.48 \\
6,950 & 0.26 & 0.30 \\
8.690 & 1.74 & 0.11 \\
9,300 & 2.05 & 0.00 \\
10,700 & 0.85 & 0.00 \\
\hline
\end{tabular}



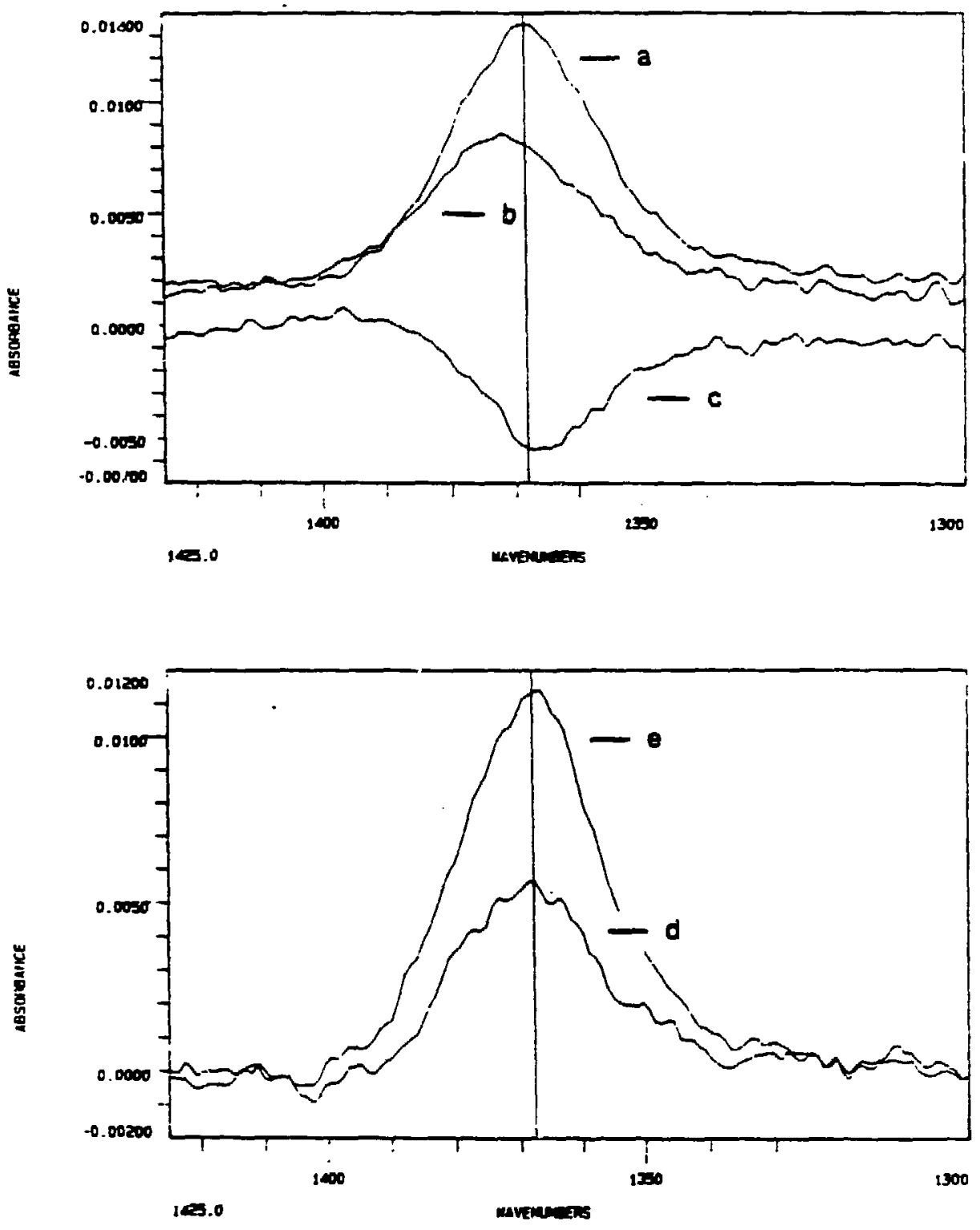

Figure III-10 Locarion of the $1369 \mathrm{~cm}^{-1}$ Absorption

A) Asymmetric depletion of the $1369 \mathrm{~cm}^{-1}$ absorption by photolysis at $3,600 \mathrm{~cm}^{-1}$. All spectra are difference spectra $(Y-X)$. (a) Maximum obtainable signal; $X(a)=$ sample after photolysis at $v<5,500 \mathrm{~cm}^{-1}, Y(a)=$ sample after photolysis at $7,600-16,500 \mathrm{~cm}^{-1}$ (b) Remaining signal: $X(b)=X(a), Y(b)=Y(a)$ followed by photolysis with $5 \times 10^{24}$ photons at $3,600 \mathrm{~cm}-1$ (c) Signal depleted; $X(c)=Y(a), Y(c)=Y(b)$

B) Symmetric growth of the $1369 \mathrm{~cm}^{-1}$ absorption on photolysis at $9,300 \mathrm{~cm}^{-1}$.

(d) Half niaximum obtainable signal; $X(d)=X(a), Y(d)=X$ (a) followed by $5 \times 10^{23}$ photons at $9,300 \mathrm{~cm}^{-1}$ (e) Maximum obtainable signal; $X(e)=X(a), Y(e)=X(a)$ followed by $5 \times 10^{25}$ photons at $9,300 \mathrm{~cm}^{-1}$ 
Table III-9 $1369 \mathrm{~cm}^{-1}$ Peak Location

Asymmetric Depletion of $1369 \mathrm{~cm}^{-1}$ Absorption

\begin{tabular}{ccc}
$\begin{array}{c}\text { Photolysis } \\
\text { Frequency } \\
\left(\mathrm{cm}^{-1}\right)\end{array}$ & $\begin{array}{c}\text { Remaining } \\
\text { Absorption } \\
\left(\mathrm{cm}^{-1}\right)\end{array}$ & $\begin{array}{c}\text { Depleted } \\
\text { Absorption } \\
\left(\mathrm{cm}^{-1}\right)\end{array}$ \\
\hline 2,800 & 1369 & 1369 \\
3,000 & 1371 & 1366 \\
3,200 & 1371 & 1366 \\
3,400 & 1372 & 1365 \\
3,600 & 1372 & 1366 \\
3,800 & 1372 & 1366 \\
4,000 & 1370 & 1369 \\
4,200 & 1369 & 1366 \\
4,400 & 1366 & 1369 \\
4,600 & 1369 & 1369 \\
4,800 & 1369 & 1369 \\
5,000 & 1369 & 1369 \\
5,200 & 1369 & 1369 \\
& & \\
\hline & &
\end{tabular}

Temperature Dependance of the $1369 \mathrm{~cm}^{-1}$ Peak Location

$\begin{array}{lllllll}\text { Temperature } & 10 \mathrm{~K} & 25 \mathrm{~K} & 50 \mathrm{~K} & 75 \mathrm{~K} & 100 \mathrm{~K} & 125 \mathrm{~K}\end{array}$

$\begin{array}{ccccccc}\begin{array}{c}\text { Peak } \\ \text { Location } \\ \left(\mathrm{cm}^{-1}\right)\end{array} & 1369 & 1369 & 1369 & 1369 & 1370 & 1372 \\ \begin{array}{c}\text { Maximum } \\ \text { Signal } \\ (\text { ABS units) }\end{array} & 0.0128 & 0.0110 & 0.0101 & 0.0072 & 0.0050 & 0.0025\end{array}$


$1369 \mathrm{~cm}^{-1}$ absorption band first. The width of this band, $27 \mathrm{~cm}^{-1}$, is likely due to different defect sites. Photolysis at frequencies above $4,000 \mathrm{~cm}^{-1}$ deplete $1369 \mathrm{~cm}^{-1}$ symmetrically; this implies similar absorption cross sections for all sites and is taken to indicate the high energy side of the absorption band.

The exact frequency of the $1369 \mathrm{~cm}^{-1}$ peak is also a function of temperature, shifting $3 \mathrm{~cm}^{-1}$ to higher frequencies at higher temperatures between 75 and $125 \mathrm{~K}$. It is not unusual for $I R$ peak frequencies to change a few wavenumbers with temperature. However, since this change in peak location accompanies a change in the maximum obtainable signal, it may be indicative of the relative stabilities of the sites contributing to the high and low frequency sides of the peak. It appears that the sites at which photoinduced IR activity is depleted by photolysis most readily do not develop long-lived photoinduced IR activity at higher temperatures.

The growth of the $1369 \mathrm{~cm}^{-1}$ peak is symmetric, this is shown in Figure II-10B.

\section{E.6 Electronic Transitions}

These data indicate that depletion of the photoinduced $\mathbf{R}$ absorptions is brought on by excitation of a transition which extends from 3,000 to $8,690 \mathrm{~cm}^{-1}$. This transition has a maximum between 3,600 and $4,200 \mathrm{~cm}^{-1}$, much like the near-IR absorption induced by heavy chemical doping or the transient photoinduced near-IR absorption asociated with the photoinduced IR absorptions. Long-lived photoinduced IR absorptions are induced by excitation of a transition with an absorption onset near $5,000 \mathrm{~cm}^{-1}$. This feature may be a band-edge absorption similar to, but at lower energy than, the absorption responsible for photoconductivity and transient photoinduced IR absorptions, or it might be a peak.

Several absorptions have been reported in this region previously. A peak at $7,300 \mathrm{~cm}^{-1}$ has been observed in photothermal deflection spectroscopy ${ }^{2}$ and direct absorption.9 A transient photoinduced absorption, not associated with the photoinduced IR absorptions has been observed at $10,900 \mathrm{~cm}^{-1}$. There is also a shoulder on the band-edge transition near 12,000 $\mathrm{cm}^{-1}, 10,11$

\section{F.1 EPR Setup}

The effect of the photoexcitation of these transitions on the spin-unpaired defect concentration was studied with EPR. Thin film samples of polyacetylene were stuffed into quartz EPR tubes. The isomer content of these samples was estimated to be the same as that of similarly prepared samples as determined by IR intensities. Sample A was $-60 \%$ trans. Sample B, originally $\sim 70 \%$ trans, was further isomerization to $>70 \%$ trans by evacuating the tube and immersing it in a $140^{\circ} \mathrm{C}$ hot oil bath for five minutes. 
The samples were cooled with a liquid transfer Heli-Trans refrigerator (Air Products, model LTR). The temperature of the coolant, helium boil-off, was monitored with a iron-doped gold/chromel thermocouple two centimeters below the sampling area. This temperature could be controlled by adjusting the helium flow rate or by heating the gas with a five-watt heating coil before it reached the sample. EPR spectra were recorded with a Varian E109 spectrometer, on a digital oscilloscope (Nicolet Eplorer 111 a). The spectra were transferred to a VAX 11,780 computer for data manipulation and display. Typical operation parameters were: field set $=3291 \mathrm{G}$, modulation amplitude $=2.5 \mathrm{G}$, and 5 $\mu W$ atts microwave power. Single scan spectra were recorded with a two minute scan time. The signal intensity was nearly linear with power in the range between 0.5 to $50 \mu \mathrm{Watts}$ at both $4.3 \mathrm{~K}$ and $20 \mathrm{~K}$.

\section{F.2 EPR Signal}

A large signal was observed at $g=2.003 \pm 0.001$. A typical spectrum is shown in Figure III-11. As expected, the signal intensity increased with decreasing temperature in accord with the Curie law. Also as previously observed the linewidth increased slightly with decreasing temperature. At $4.3 \mathrm{~K}$ the linewidths $\left(\Delta \mathrm{H}_{\mathrm{p}-\mathrm{p}}\right)$ of samples $A$ and $B$ were $7.6 \mathrm{G}$ and $5.8 \mathrm{G}$, respectively. Sample A's higher cis content was reflected in its wider linewidth.

The samples were photolysed with the heat lamp through a $5 \mathrm{~mm}$ germanium plate ( $v<5500 \mathrm{~cm}^{-1}$ ) and through the Coming Glass water filter combination $(7,600<v<$ $16,500 \mathrm{~cm}^{-1}$ ). During photolysis in either frequency range the EPR signal narrowed and decreased dramatically, 50 to 75\%, in intensity. This decrease in intensity and linewidth is consistent with a $20^{\circ}$ increase in temperaturc; although, the thermocouple, which measured the temperature of the helium before it cooled the sample, indicated a constant temperature during photolyses. About five minutes after each photolysis thermal equilibrium was reestablished and the initial signal intensity was nearly recovered. After the sample temperature was reestablished the intergrated signal intensity after photolysis through the germanium plate $\left(v<5,500 \mathrm{~cm}^{-1}\right)$ was 4 to $7 \%$ smaller than after higher energy $(7,600$ $16.500 \mathrm{~cm}^{-1}$ ) photolysis.

Surrounding sample B with dried distilled degassed hexanes increased the sample's heat capacity and eliminated most of the sample heating during photolysis. The solvent also served as a medium for thermal contact to a second iron-doped gold/chromel thermocouple used to monitor the temperanure in the sampling region to one twentieth of a degree. With this precision in temperature measurement, near $4 \mathrm{~K}$ a one percent variation in integrated signal intensity can be attributed to the Curie law dependence. Comparing 


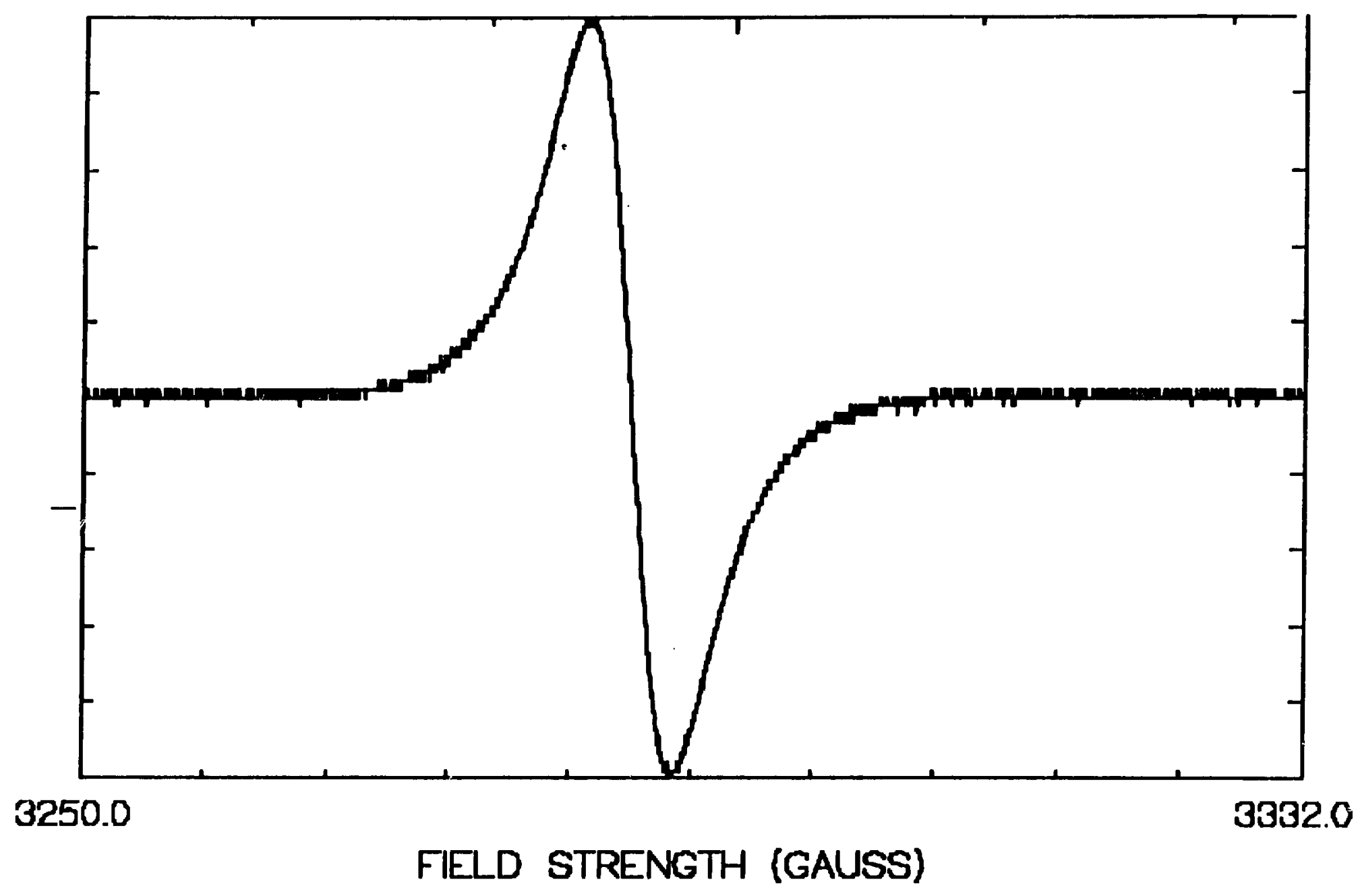

Figure 11I-11 Typical EPR Spectrum 
only spectra taken at the same temperature, five to ten minutes after photolysis, the integrated intensity of the signal after photolysis through $\mathrm{CG}+\mathrm{H}_{2} \mathrm{O}\left(7,600-16,500 \mathrm{~cm}^{-1}\right)$ was 5.7 to $5.8 \%$ greater than after photolysis through the $\mathrm{Ge}\left(v<5,500 \mathrm{~cm}^{-1}\right)$ filter. The percent difference decreased in time to less than $1 \%$ half an hour after the photolyses.

The photoinduced change in the integrated intensity of the EPR signal indicates that the population of defects with unpaired spins can be increased with upper-mid-gap (7,600 $16,500 \mathrm{~cm}^{-1}$ ) photons. Correlating this change with photoinduced changes observed in the $\mathbb{R}$, it appears either that both charged defects and defects with unpaired spin, or that defects with both an unpaired spin and a charge, are created by the higher energy $(7,600$ $\left.16,500 \mathrm{~cm}^{-1}\right)$ photolysis. In either case, the effect(s) are negated by photolysis with lower energy $\left(v<5,500 \mathrm{~cm}^{-1}\right)$ light. 


\section{References}

1 T. Ito, H. Shirakawa, S. J. Ikeda, Polym. Sci., Polym. Chem. Ed. 12, 11, (1974)

2 G. L. Baker, J. A. Shelburne III, F. S. Bates, J. Am. Chem. Soc. 108, 7377 (1986)

3 K. C. Herr, P. B. Forney, G. C. Pimentel, Applied Optics, 11(3), 493 (1972)

$4 \quad$ H. H. Wieder, Laboratory Notes on Electrical and Galvanomagnetic Measurements, Materials Science Monographs, 2 (Elsevier Scientific Publishing Company, Amsterdam, 1979) chap.1

5 Y.-W. Park, A. J. Heeger, M. A. Druy, A. G. MacDiarmid, J. Phys. Chem. 73(2), 946 (1980)

6 Z. Vardeny, J. Orenstein, G. L. Baker, Phys. Rev. Lett. 5n(25), 2032 (1983)

7 J. C. W. Chien, F.E. Karasz, G.E. Wnek Nature (london) 285, 390 (1980)

8 G. B. Blanchet, C. R. Fincher, A. J. Heeger, Phys. Rev. Lett. 51(23), 2132 (1983)

9 N. Suzuki, M. Ozaki, S. Etemad, A. J. Heeger, A. G. Macdiarmid, Phys. Rev. Lett. 45, 1209 (1980)

10 H. Eckhardt, R. R. Chance, Phys. Chem. 79(11), 5698 (1983)

11 S. Etemad, G. L. Baker, C. B. Roxlo, B. R. Weinberger, J. Orenstein, Mol. Cryst. Liq. Cryst. 117, 275 (1985) 


\section{Chapter IV.}

\section{A Definitions}

The results presented in Chapter III are discussed in this chapter. The previously reported results presented in Chapter I and interpretations of those results are used as a point of departure for the interpretation of the new results. For the purpose of this discussion some of definitions of terms used in Chapter I have been modified.

Non-bond-alternating defects with an odd number of carbons are referred to as solitons. Even carbon non-bond-alternating defects are referred to as polarons. It is expected that solimns can exist with positive, negative, or no charge. Only charged polarons can exist. Because solitons extend over an odd number of carbons, the reverse spin/charge relationship applies; thus, neutral solitons have an unpaired spin and charged solitons do not. Since polarons are ven carbon defects, they have a normal spin/charge relationship such that both positively and negatively charged polarons have an unpaired spin. EER measurements can be used to detect defects with unpaired spins.

Nothing will be assumed about the solitary wave/particle-like properties of these defects. Slight variations in characteristics of the individual defects, such as their length, could in part account for the breadth of the $\mathbb{R}$ absorptions and the distribution of states in the electronic transitions. This would not be possible if the length of these defects were uniquely determined as it should be for particle-like defects. Experimental results and theoretical calculations agree that defects in trans-polyacetylene are mobile down to $30 \mathrm{~K}$. However, attempts are made not to use this result since it has lead to the seemingly unrealistic result that the efrective mass of these defects is similar to that of an electron.

Calculations suggest that the electronic states associated with solitons are located $5,000 \mathrm{~cm}^{-1}$ above the valence band and that those associated with positively and negatively charged polarons are $3,200 \mathrm{~cm}^{-1}$ above the valence band, or below the conduction band, respectively. These suggestions come from the quantum mechanical treatment of the defects as solitary waves in a two-phase system and will be accepted only as reasonable possibilities.

Strong IR absorptions near 1370, 1290 and $500 \mathrm{~cm}^{-1}$ are considered to be the signature of charged defects. The absorptions at 1370 and $1290 \mathrm{~cm}^{-1}$ are believed to be vibrational modes of charged non-bond-alternating defects. The large extinction coefficients for these modes are believed to be due to vibronic enhancement from the movement of the excess charge. The suggestion that absorptions at these frequencies are common to solitons and polarons, with polarons having additional absorptions and higher extinction coefficients, ${ }^{1}$ is recognized as a reasonable possibility because of the similar 
st uctures of these defects. The assignment of these bands, however, is viewed with a little more skepticism. The modes to which the absorptions at 1370 and $1290 \mathrm{~cm}^{-1}$ are currently assigned involve considerable $\mathrm{C}=\mathrm{C}$ and $\mathrm{C}-\mathrm{C}$ stretching, ${ }^{1}$ but the isotope shift, 1.3, indicates a strong contibution from a $\mathrm{C}-\mathrm{H}$ bending motion, possibly the out-of-plane bend.

The origin of the low frequency mode, at $832 \mathrm{~cm}^{-1}$ for dopant-induced defects and near $500 \mathrm{~cm}^{-1}$ for photoinduced defects, is also questionable. Despite their similar and distinctive shapes, it is not entirely clear that they have the same origin. The isotope shifts and frequencies for these absorptions are very different. The difference in location has been attributed to the difference in she pinning potentials created by dopani counter-ions and oppositely charged defects. Yet, the difference in isotope shift, 1.24 in dopant-induced and 1.05 in photoinduced, has not been addressed. While these isotope shifts may be somewhat inaccurate, because of the breadth of the absorption, they can not be completely ignored. Thus, the assignment of these absorptions is left open to discussion.

\section{B.1 Chemically Induced Defects}

Previous work on the chemical doping process shows different behaviors in polyacetylene at different doping levels. At low doping levels, $y<0.001$ for $\left(\mathrm{CHZ}_{\mathbf{y}}\right)_{\mathbf{x}}, \mathbb{R}$ activity is induced, ${ }^{2}$ the thermopower coefficient is large, ${ }^{3}$ and there is no change in the EPR signal intensity ${ }^{4}$ All the unpaired spins obey the Curie Law acting like isolated organic tree radicals. There is a $0.3 \mathrm{eV}$ activation enrrgy to electrical conductivity. Conduction in lightly doped, fairly resistive, polyacerylene is believed to proceed via electrons hopping from one defect site to another. ${ }^{3}$ In the intermediate dopant level range, $0.001<y<0.1$, some defects overlap each other creating small metallic islands. ${ }^{5}$ These islands are at varying distances from each other and some hopping between islands is required for bulk conductivity. This explains the faster increase in a.c. conductivity than in d.c. conductivity with increasing dopant concentrations. Increasing dopant concentration, in this concentration range, increases the conductivity and the intensity of the $\mathbb{R}$ absorptions, atributed to charged solitons, while decreasing the themopower coefficient, the activation energy for conductivity, and the number of Curie law spins, which all reach zero at $y=0.1 .3$ When $y=0.1$ the defect states, with halfwidths $l=5$ carbon bond lengths, all overlap. The Curie law EPR signal has been replaced by an EPR signal with a Dysonian line shape which obeys the Pauli law, characteristic of metals. At this doping level the conductivity, tending towards metallic behavior, does not decrease significantly with increasing temperature.

Some researchers report a near-IR absorption at $6,000 \mathrm{~cm}^{-1}$ at low doping levels, shifting to $4,000 \mathrm{~cm}^{-1}$ at higher doping levels. ${ }^{6}$ Others report only an absorption near 
$4,000 \mathrm{~cm}^{-1}$ at all doping levels. ${ }^{7}$ This discrepancy is probably due to different doping techniques. Rapid doping or doping of dense samples can result in inhomogeneous doping. The location of the near-IR absorption is the only clear indicator of inhomogeneous doping at low dopant concentrations. Other properties, such as conductivity, display similar "intermediate" behaviors for both individual defects and tiny metallic islands isolated in the bulk semiconductor, bond-alternated polyacetylene.

When polyacetylene is doped at low temperatures ( 50 to $150 \mathrm{~K}$ ) the near-IR absorption is at particularly low energy, 4,000 to $3,000 \mathrm{~cm}^{-1}$ (this work). This is not surprising since inhomogeneous doping is expected when dopant diffusion is limited. The conductivity measurements also clearly indicate inhomogeneous doping.

The low temperature doping experiments described in Chapter III were designed primarily to determine the temperature below which doping would not occur thermally. There was, however, a more interesting result contained in this information. The doping temperatures observed for the various dopants were not related to the melting points of the dopants. This implies that although there is poor diffusion thermodynamics, not kinetics, determine the thermal barrier for the doping process. This observation offers support for and insight into the popular charge-transfer picture of doping.

Although it is not often discussed in much detail it is generally assumed that chemical doping proceeds via a charge transfer reaction. The charge transfer process for $p$ doping is illustrated in Figure IV-1. In the charge transfer doping reaction an electron is removed from polyacetylene by an acceptor molecule. The polymer chain undergoes a structural rearrangement to stabilize the charge, creating a region in which there is no bondlength-altemation, a soliton or polaron. Charge transfer is possible only when the electrostatic atraction between the dopant ion and the charged defect on the polymer, $V(+/-)$, provides enough energy to make up the large difference between the ionization potential of the polymer and the electron affinity of the acceptor. Figure IV-1 pictures polaron formation; the charge transfer process for soliton formation is essentially the same. The difference is that the formation of a charged soliton requires the simultaneous formation of a second soliton on the same chain, either a neutral soliton with unpaired spin or a charged soliton from a concurrent charge-transfer reaction. The direction of the bondalternation in the surrounding polymer is reversed by a single soliton, thus a second soliton is required to retum the bond-alternation to its original direction. 


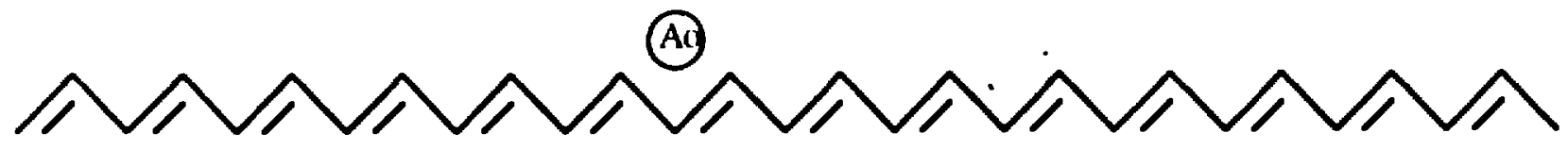

$\downarrow^{A}$

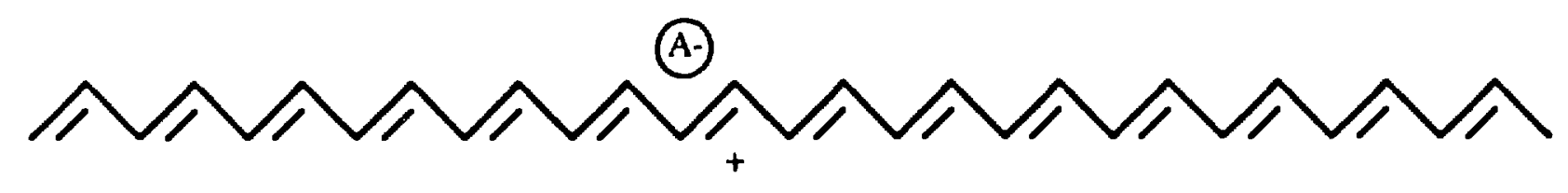

$\downarrow$ B)
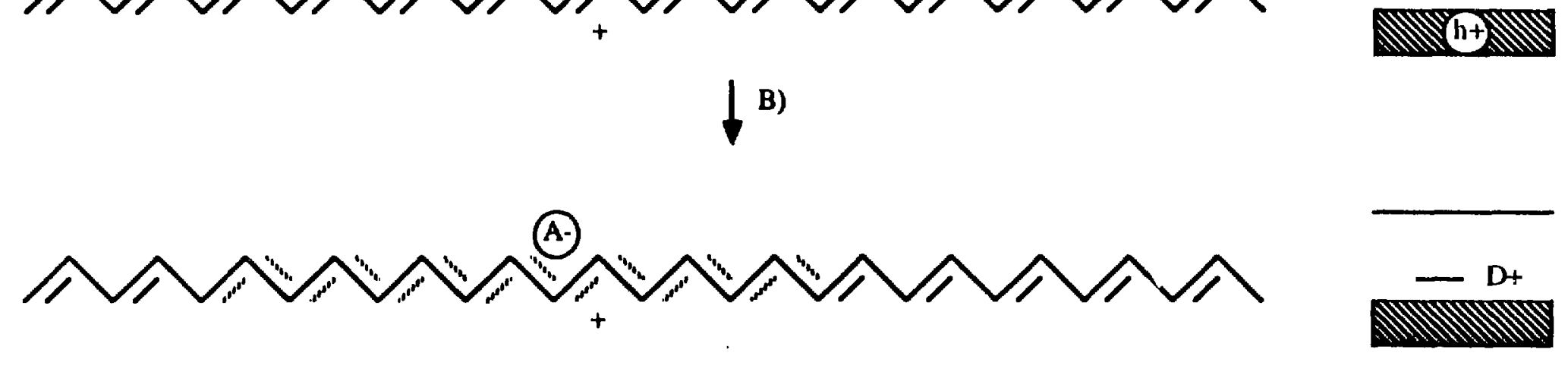

Figure IV-1 P-Type Charge Transfer Doping

A) An electron is transfierred from the polymer chain to an acceptor molecule, creating a hole in the valence tand. B) The polymer accommodates the hole in the valence band by adopting a non-bond-altermating structure over $-15 \mathrm{C}$ - $\mathrm{C}$ bond lengths. The defect is bound electrostatically to the reduced acceptor ion localizing it in the bulk semi conductor. The electronic state associaled with this defect is in the band-gap. 
The following reactions have been proposed for chemical doping with sodium naphthalide, ${ }^{8}$ bromine, ${ }^{9}$ and arsenic pentafluoride: 10

$$
\begin{gathered}
(\mathrm{CH})_{x}+\mathrm{Na}^{+} \text {Naphthalene }-(\mathrm{CH})_{x}^{-} \mathrm{Na}^{+}+\text {Naphthalene } \\
2(\mathrm{CH})_{x}+3 \mathrm{Br}_{2} \rightarrow 2(\mathrm{CH})_{x}+2 \mathrm{Br}_{3}^{-} \\
(\mathrm{CH})_{x}+2 \mathrm{AsF}_{5} \rightarrow(\mathrm{CH})_{x}+\mathrm{AsF}_{6}^{-}+\mathrm{AsF}_{3(\mathrm{~g})}
\end{gathered}
$$

$N$-doping with sodium naphthalide does not involve the direct transfer of charge between the dopant and polyacetylene. The main charge transfer reaction occurs when the sodium naphthalide solution is prepared. An electron is removed from the sodium atom and added to the naphthalene molecule. The energy provided by the electrostatic attraction between the sodium and naphthalide ions makes up the energy difference between the ionization potential of sodium and the electron affinity of naphthalene. When polyacetylene is washed with the sodium naphthalide solution an electron is transferred from the naphthelene to the polymer, which has an electron affinity greater than that of naphthalene. It can be assumed that the electrostatic attraction between a sodium and a naphthalide ion is similar to that between a sodium ion and a negatively charged, delocalized, non-bondaiternating defect on polyacetylene.

The enthalpies of the proposed reactions for chemical doping with sodium naphthalide and bromine are calculated in Appendix 1. The ionization energy ${ }^{11}$ and electron affinity 12 of cyclooctatetraene were used to approximate those for polyacetylene. This prototype molecule was chosen because like polyacetylene it undergoes a structural transformation from a bond-altemated to a non-bond-altemated structure to stabilize charge. The electrostatic attraction between the charge defect and counter-ion was calculated for point charges at a separation of $2 \AA .13$ The enthalpy of reaction for iodine doping, assuming the same reaction proposed for bromine, is also calculated in Appendix 1, as are those for doping with $\mathrm{HCl}$ and $\mathrm{HBr}$, assuming each of the following possible reactions :

$$
\begin{aligned}
& 2(\mathrm{CH})_{x}+4 \mathrm{HX} \rightarrow 2(\mathrm{CH})_{x}^{+}+2 \mathrm{HX}_{2}^{-}+2 \mathrm{H}_{2} \\
& 2(\mathrm{CH})_{x}+6 \mathrm{HX} \rightarrow 2(\mathrm{CH})_{x}^{+}+2 \mathrm{X}_{3}^{-}+3 \mathrm{H}_{2}
\end{aligned}
$$

Reaction (D) is endothermic for $\mathrm{HCl}$. Since chemical doping with both $\mathrm{HBr}$ and $\mathrm{HCl}$ has been observed even at low temperatures (down to $60 \mathrm{~K}$ ), reaction (D) can be eliminated. 


\section{B.2 Chemical Trapping of Photoinduced Defects}

The photoexcitation of polyacetylene with light $v>9,000 \mathrm{~cm}^{-1}$ is believed to create charged defects similar to those created by chemical doping. These transient, photoinduced, IR active defects have been observed to live for a few milliseconds. ${ }^{14}$ The creation of these defects should open up new, excited-state reaction pathways for chemical doping. An electron could be transferred from a negatively charged transient photoinduced defect to the acceptor molecule. The enthalpy of this reaction would be simply the difference in the electron affinities of the acceptor and the polymer, as in the sodium naphthalide $n$-doping reaction. The oppositely charged defect could then migrate to the vicinity of the acceptor ion and bind to it electrostatically. In the soliton case, the positive charge can hop from the photoinduced positively charged soliton to the neutral soliton created when the photoinduced negatively charged soliton is oxidized. Figures IV-2 and IV-3 illustrate the processes proposed for the chemical stabilization of photoinduced solitons and polarons. It was anticipated that charge transfer reactions between long-lived, charged, photoinduced defects and potential chemical dopants would occur with a lower activation barrier, thus proceeding at an observable rate at lower temperatures than chemical doping of ground state polyacetylene.

The heats of reaction for the chemical doping of photoexcited polyacetylene (chemical stabilization of photoexcitations in polyacetylene) with $\mathrm{HCl}$ and $\mathrm{HBr}$ are calculated in Appendix 1 assuming the formation of both charged solitons and polarons:

$$
\begin{aligned}
& \left.S^{-}+S^{+}+2 \mathrm{HX} \rightarrow \mathrm{S}^{0}+{ }_{[} S^{+} \mathrm{HX}_{2}^{-}\right]+1 / 2 \mathrm{H}_{2} \\
& \mathrm{P}^{-}+\mathrm{P}^{+}+2 \mathrm{HX} \rightarrow\left[\mathrm{P}^{+} \mathrm{HX}_{2}^{-}\right]+1 / 2 \mathrm{H}_{2}
\end{aligned}
$$

When the charge is removed from a polaron, even carbon defect, the polymer chain supporting the polaron becomes subject to the Peierls instability. The length of polymer which had supported the polaron reverts to a bond-altemated structure. Isolated solitons, odd carbon defects, do not undergo this non-boid-altemated to bond-alternated structural transformation when they lose their charge. Thus the electron affinity of naphthalene was used to approximate that of a neutral soliton, while that of cyclooctatetraene was used for an even carbon siretch of polyacetylene which can support a charged polaron.

The calculated exothermicities for both of these reacrions are much greater than those for the chemical doping of polyacetylene with $\mathrm{HBr}$ and $\mathrm{HCl}:-407.0$ or $-386.6 \mathrm{vs}$. $-45.6 \mathrm{kcal} /$ mole for $\mathrm{HCl}$ and -420.6 or -400.2 vs. $-59.4 \mathrm{kcal} / \mathrm{mole}$ for $\mathrm{HBr}$. Yet 


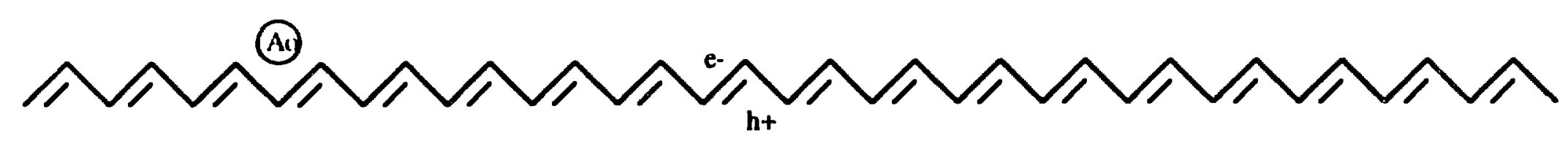

$\downarrow$ A)

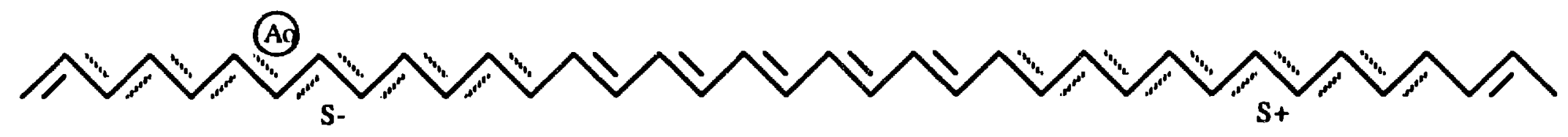

$\downarrow$ B)
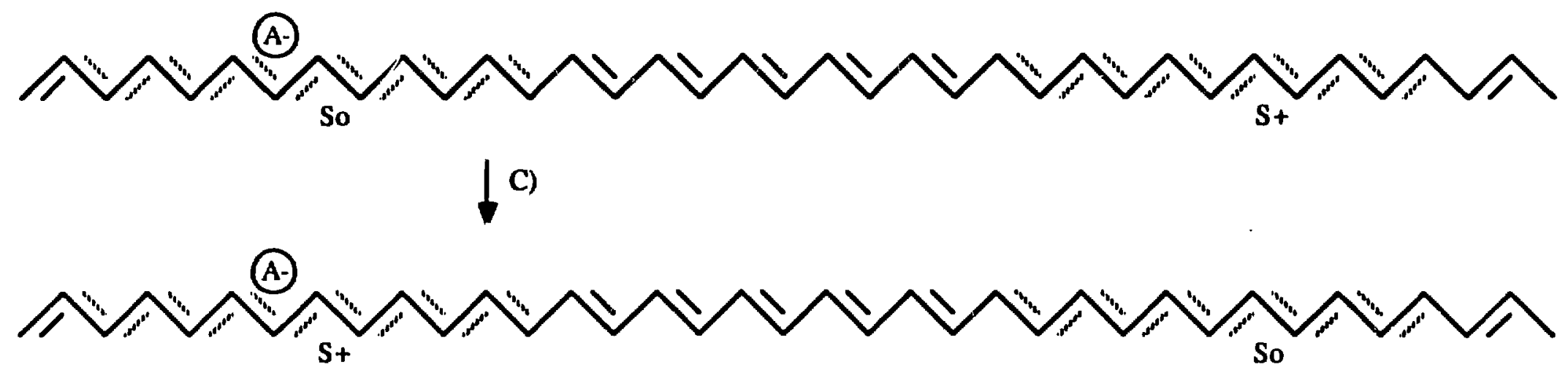

Figure IV-2 P-Type Chemical Stabilization of Photoinduced Charged Solitons

A) A photoinduced electron-hole pair relaxes to a pair of charged solitons. B) An electron is transferred from a negatively charged photoinduced soliton to an acceptor molecule. C) The charge on the oppositely charged soliton hops to the neutral soliton and is electrostatically bound to the oxidized acceptor ion. 


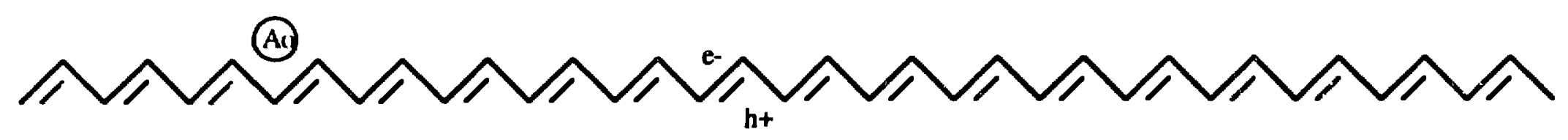

$f^{\text {A) }}$

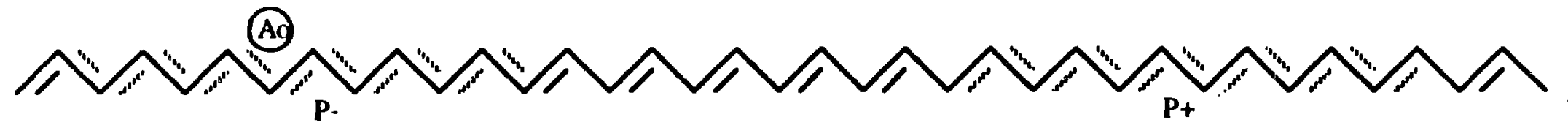

$\downarrow^{\text {B) }}$

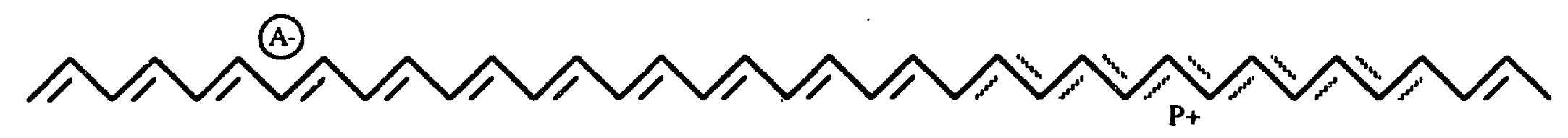

1 c)

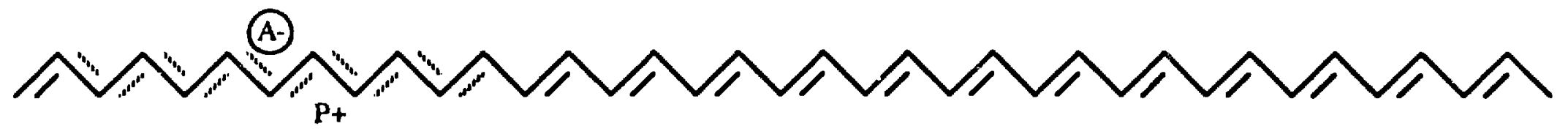

Figure IV-3 P-Type Chemical Stabilization of Photoinduced Charged Polarons

A) A photoinduced electron-hole pair relaxes to a pair of charged polarons. B) An electron is transferred from a negatively charged photoinduced polaron to an acceptor molecule. The chain where the negatively charged polaron had been relaxes to a bond-alternated structure. C) The oppositely charged polaron migrates towards the oxidized acceptor ion and is electrostatically bound to it. 
photoexcitation was not observed to alter the temperature required for doping with either $\mathrm{HCl}$ or $\mathrm{HBr}$. The photochemical reaction is either greatly hindered for some kinetic reason or has an activarion energy equal to or greater than the chemical reaction.

The stepwise reaction mechanisms initially proposed for chemical doping of polyacetylene and for the chemical stabilization of photoinduced defects in polyacetylene with $\mathrm{HCl}$ are as follows ( $\Delta \mathrm{H}$ in kcals/mole):

Net Rxn. (C)

$2(\mathrm{CH})_{\mathrm{x}}+4 \mathrm{HCl} \rightarrow 2\left[(\mathrm{CH})_{\mathrm{x}}+\mathrm{HCl}_{2}^{-}\right]+\mathrm{H}_{2}$

$\Delta \mathrm{H}=-45.6$

$i$

$2\left\{(\mathrm{CH})_{\mathrm{x}}+\mathrm{HCl} \rightarrow\left[(\mathrm{CH})_{\mathrm{x}}+\mathrm{Cl}^{-}\right]+\mathrm{H} \cdot\right\}$

$\Delta \mathrm{H}=2(36.9)$

ii

$2 \mathrm{H} \cdot \rightarrow \mathrm{H}_{2}$

$\Delta \mathrm{H}=-103.2$

iii

$2\left\{\mathrm{Cl}^{-}+\mathrm{HCl} \rightarrow \mathrm{HCl}_{2}^{-}\right\}$

$\Delta \mathrm{H}=2(-8.1)$

Net Rxn. (E)

$2 S^{-}+2 S^{+}+4 \mathrm{HCl} \rightarrow 2 S_{0}+2\left[S^{+} \mathrm{HCl}_{2}^{-}\right]+\mathrm{H}_{2}$

$\Delta \mathrm{H}=-407.0$

$i$

ï

iii

iv

Net Rxn.

(F)

$2 \mathrm{P}-+2$

$2\left[\mathrm{P}^{-}+\mathrm{HCl} \rightarrow(\mathrm{CH})_{x}+\mathrm{Cl}^{-}+\mathrm{H}^{\cdot}\right]$

$2\left\{\mathrm{~S}^{-}+\mathrm{HCl} \rightarrow \mathrm{S}_{\mathrm{o}}+\mathrm{Cl}^{-}+\mathrm{H}^{\bullet}\right\}$

$\Delta H=2(22.2)$

$2 \mathrm{H} \cdot \rightarrow \mathrm{H}_{2}$

$\Delta \mathrm{H}=-103.2$

$2\left[\mathrm{Cl}^{-}+\mathrm{HCl} \rightarrow \mathrm{HCl}_{2}^{-}\right]$

$\Delta \mathrm{H}=2(-8.1)$

$2\left\{\left[\mathrm{~S}_{\mathrm{o}} \mathrm{HCl}_{2}^{-}\right]+\mathrm{S}^{+} \rightarrow\left[\mathrm{S}^{+} \mathrm{HCl}_{2}^{-}\right]+\mathrm{S}_{\mathrm{o}}\right\}$

$\Delta \mathrm{H}=2(-166)$ $i$

$\ddot{i}$

iii

iv

$$
\begin{gathered}
2 \mathrm{H}-\rightarrow \mathrm{H}_{2} \\
2\left[\mathrm{Cl}^{-}+\mathrm{HCl} \rightarrow \mathrm{HCl}_{2}^{-}\right) \\
2\left\{\mathrm{HCl}_{2}^{-}+\mathrm{P}^{+} \rightarrow\left[\mathrm{P}^{+} \mathrm{HCl}_{2}^{-}\right]\right\}
\end{gathered}
$$

$\Delta \mathrm{H}=-386.6$

$\Delta \mathrm{H}=2(32.4)$

$\Delta \mathrm{H}=-103.2$

$\Delta H=2(-8.1)$

$\Delta H=2(-166)$

In each of these reaction mechanisms step $i$ is the rate limiting step. The predicted activation energy for the chemical doping reaction is almost twice that for chemical stabilization of photoinduced solitons (C). The activation energy predicted for chemical stabilization of photsinduced polarons is very close to that predicted for chemical doping. All of these predicted activation energies are much higher than can be expected for reaction(s) which take place at an observable rate at $50 \mathrm{~K}$. The chemical doping of polyacetylene with $\mathrm{HCl}$ has been observed at $50 \mathrm{~K}$. This suggests that the actual activation energy for chemical doping is between 3 and $12 \mathrm{kcal} / \mathrm{mole}$. To conform to this fact, the 
proposed doping mechanisms must be modified such that steps $i$ and $i i$ occur simultaneously.

The rate at which the chemical doping reaction occurs depends on the temperature, $T$, activation energy, $E_{a}$, and a pre-exponential fractor, $A_{c}$. In the chemical doping experiment the prospective dopant was frozen onto the surface of the solid polymer, therefor, all prospective reaction sites were fixed. In the stabilization experiment there was a steady state concentration of photoinduced defects of $5 \times 10^{17} \mathrm{~cm}^{-3} .14$ Only these defects can serve as reactive sites in the proposed chemical stabilization of photoinduced defects reactions. Thus, the pre-exponential factor for the reactions proposed for the chemical stabilization of photoinduced defects, $A_{p}$, should be smaller than that for the chemical doping reaction. The density of polyacetylene is 4 grams cm$~^{-3} .15$ Thus the density of C-H units is $2 \times 10^{23} \mathrm{~cm}^{-3}$. Each defect is about 10 carbons long thus the fraction of the carbons involved in defects is $2.5 \times 10^{-5}$. If the reaction probabilities are otherwise the same, then the pre-exponential factor for the stabilization of photoinduced defects reactions, $A_{p}$, is $2.5 \times 10^{-5} A_{c}$. Assuming these pre-exponential factors, the relative rates of reaction for reactions (C), (E), and (F) at 50 and $30 \mathrm{~K}$ are calculated in Appendix 2. The activation energies used in these calculations are the endothermicities of step $i$ in the original stepwise mechanisms scaled down proportionally to a reasonable range, 3 to $12 \mathrm{kcal} / \mathrm{mole}$.

The relative rates calculated for the chemical doping of polyacetylene with $\mathrm{HCl}$ at 50 and $30 \mathrm{~K}$, assuming $3,6,9$, and $12 \mathrm{kcal} / \mathrm{mole}$ activation energies, are all sufficiently different that the reaction could proceed at a perceptible rate at $50 \mathrm{~K}$ and not at $30 \mathrm{~K}$. When higher activation energies are assumed the relative rate of soliton stabilization at either temperature is much greater than the chemical reaction. If the activation energy for the chemical reaction is as low as $3 \mathrm{kcal} / \mathrm{mole}$ the stabilization of photoinduced defects is not a comperitive process. If the activation energy of the chemical reaction is assumed to be between 6 and $9 \mathrm{kcal} / \mathrm{mole}$ the chemical stabilization of photoinduced solitons could be observed at lower temperatures than the chemical reaction. If the activation energy of the chemical reaction were as high as $12 \mathrm{kcal} / \mathrm{mole}$, which is doubtful, the chemical stabilization of either photoinduced solitons or polarons could be observed below the temperature at which chemical doping is observed. Since no decrease in the temperature required for reaction was observed during photolysis either the the activation energy for the chemical reaction is less than $6 \mathrm{kcal} / \mathrm{mole}$ or it is less than $9 \mathrm{kcal} / \mathrm{mole}$ and photoinduced defects are polarons. 


\section{C.1 Observed Energy Levels in the trans-Polyacetylene Band gap}

Previous workers ${ }^{16}$ have identified the band-gap transition in trans-polyacetylene at $15,700 \mathrm{~cm}^{-1}$ with its onset at $11,300 \mathrm{~cm}^{-1}$. A very weak shoulder on this absorption near $11,000 \mathrm{~cm}^{-1}$ has been resolved with photoacoustic spectroscopy 17 and in direct absorption spectroscopy on compensated samples. 18 There are several regions of interest below the band gap.

A peak at 7,300 $\mathrm{cm}^{-1}$ has also been observed in direct absorption ${ }^{19}$ and photothermal deflection spectroscopy. 20 Baker attributes this peak to inadvertent oxidation, whereas, Suzuki attributes it to neutral defects. Inadvertent doping seems more likely since other investigations, done with similar sensitivity, ${ }^{17}$ have failed to observe this peak. This absorption might be due to adventitious $n$-doping in uncompensated samples caused by residual catalyst. A broad absorption grows in near $7,000 \mathrm{~cm}^{-1}$ on chemical doping, and shifts toward $4,000 \mathrm{~cm}^{-1}$ with increased dopant concentration. 6

The onset of photoconductivity in undoped polyacetylene is at $9,000 \mathrm{~cm}^{-1.21}$ The photoconductive response increases rapidly to $12,000 \mathrm{~cm}^{-1}$ where it levels out. In this region the photoconductive gain is extremely temperature dependent, dropping with decreasing temperature, and is not detectable below $150 \mathrm{~K}$. There is also a peak in the photoconductive response at $7,300 \mathrm{~cm}^{-1}$. This peak is noticeably less dependent upon temperature than the higher energy feature and is most likely due to the above mentioned light adventitious doping peak.

The excitation spectrum for photoinduced IR absorption 22 is very similar to that for photoconductivity between $9,000 \mathrm{~cm}^{-1}$ and $12,000 \mathrm{~cm}^{-1}$. In this frequency region, the intensity of the photoinduced absorptions decreases slightly with increasing temperature to $150 \mathrm{~K}$. Above this temperature the photoinduced absorptions lose intensity rapidly, giving way to photoconductivity.

Two broad, transient, electronic transitions have been observed during photoexcitation above the band gap. The lower energy photoinduced peak at $3,900 \mathrm{~cm}^{-1}$ shows the same temperature dependence as the transient photoinduced IR absorptions near 1370, 1290 and $500 \mathrm{~cm}^{-1} .14$ The higher energy transient photoinduced absorption, a structured peak near $11,000 \mathrm{~cm}^{-1}$, decreases with increasing temperature much more rapidly than the low energy peak. Above $150 \mathrm{~K}$, the higher energy peak is bleached rather than enhanced by above-band-gap photoexcitation. This absorption is bleached at all temperatures during below-band-gap photoexcitation $\left(>9,000 \mathrm{~cm}^{-1}\right) .23$

The decay of the higher energy absorption, photoinduced by above-gap light, has been observed from 0.5 to $100 \mathrm{ps.}^{24}$ In this time range the signal intensity is roughly proportional to the square root of time. The decay is ten times as fast at $20 \mathrm{~K}$ as at room 
temperature. The time evolution of the low energy, transient photoinduced absorption has been observed with lower resolution from 0 to $1000 \mathrm{ps} .{ }^{25}$ The initial absorption decays within ten picoseconds at $25 \mathrm{~K}$ and at room temperature. At $25 \mathrm{~K}$ the absorption reappears between 30 and 50 ps. The second appearance is much more pronounced when Shirakawa polyacetylene is used than when Baker polyacetylene, with fewer neutral defects, is used.

The assignments which are used to explain these experimental data involve several mid-gap states (Figure IV-4). Light and adventitious doping produce an absorption at $7,300 \mathrm{~cm}^{-1}$ (a), very near the middle of the band gap. This absorption is associated with a charged defect because it is affected by charge-transfer chemistry. Light doping does not change the EPR spectra of polyacetylene, thus the mid-gap level is assigned to charged spinless defects, i.e. charged solitons, at low concentration. Heavy chemical doping and photoexcitation produce an absorption near $4,000 \mathrm{~cm}^{-1}$ (b). The photoinduced defect involved in this transition was determined to be spinless. The EPR spectra of heavily doped polyacetylene is complicated by the transition to metallic behavior, converting from Curie law (organic free electrons) to Pauli law (metallic free electrons) spins. The defects produced by heavy doping are assumed to be the same as those produced by photoexcitation: charged solitons at relatively high density. The shoulder on the band-gap . absorption edge and the transient photoinduced or photobleached peak at $11,000 \mathrm{~cm}^{-1}$ are assigned to the same state (c). The state involved in this transition is believed to be in the upper half of the band gap because there is vibrational structure on its high frequency side. This state is believed to be neutral because there is no IR activity or charge-transfer chemistry associated with it. Neutral polarons are unstable, thus the state at $11,000 \mathrm{~cm}^{-1}$ is assigned to a neutral soliton.

Since the band gap onset is $4,400 \mathrm{~cm}^{-1}$ below its absorption maximum, the onset for mid-gap transitions should be $2,200 \mathrm{~cm}^{-1}$ below their maxima. The photoconductive response curve and photoinduced absorption excitation spectra can be viewed as a superposition of the $11,000 \mathrm{~cm}^{-1}$ and band-gap absorptions, with more weight on the $11,000 \mathrm{~cm}^{-1}$ absorption. Photoexcitation of the $11,000 \mathrm{~cm}^{-1}$ transition promotes an electron from the valence band to a neutral soliton. The polymer responds to the hole left in the valence band with the formation of a charged bond-altemation defect. This defect, depending on the temperature, either acts as a charge carrier ( $T>150 \mathrm{~K}$ ) or can be detected in the IR and near-IR. The $11,000 \mathrm{~cm}^{-1}$ absorption has its onset near $9,000 \mathrm{~cm}^{-1}$, consistent with the photoconductive response curve and photoinduced absorption excitation spectra. Excitation into the band gap (onset $11,300 \mathrm{~cm}^{-1}$ ) is capable of creating electrons, as well as hole, both of which are capable of forming charged defects that can act as charge carriers. Photoexcitation of the band-gap transition should produce considerably more 


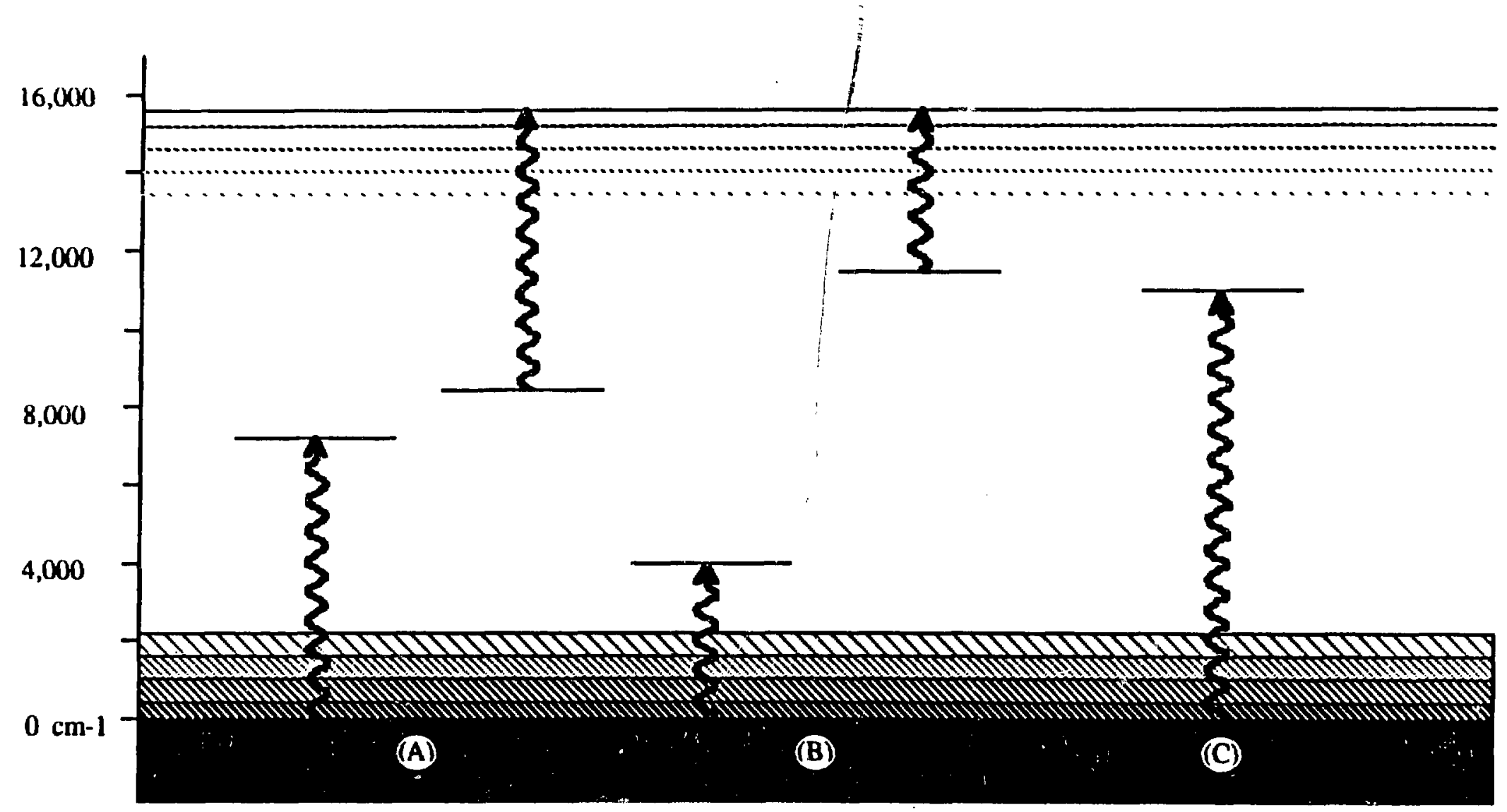

Figure IV-4 Observed Transitions in trans-Polyacetylene

A) $7,300 \mathrm{~cm}^{-1}$ observed in lightly and adventitiously doped samples; attributed to charged solitons at low concentration B) $4,000 \mathrm{~cm}^{-1}$ observed in heavily doped samples and during photolysis $\left(U>9,000 \mathrm{~cm}^{-1}\right)$; attributed to charged solitons at high concentration $\left.C\right)$ $11,000 \mathrm{~cm}^{-1}$ Observed as a shoulder on the band edge transition also photobleached $\left(9,000 \mathrm{~cm}^{-1}<v<14,000 \mathrm{~cm}^{-1}\right)$ and photoinduced $\left(v>14,000 \mathrm{~cm}^{-1}\right)$; attributed to neutral solitons 
charged defects than of the $11,000 \mathrm{~cm}^{-1}$ peak, but the effect appears to saturate at a concentration near that produced with $12,000 \mathrm{~cm}^{-1}$ light. This may be caused by the possible recombination of positively and negatively charged defects.

\section{C.2 Proposed Mechanisms for The Photoproduction of Transient Defects}

Experimental investigations of photoinduced charged defects were originally inspired by Su's prediction 26 that photoinduced electrons and holes should be unstable with respect to the formation of charged structural defects. Electron-hole pairs produced on a single chain were predicted to relax to oppositely charged soliton pairs. Electrons and holes on separate chains were predicted to form charged polarons. The ability to form these defects is an intrinsic property of the bond-alternated trans-polyacetylene structure. Thus, the direct formation of charged defects from photoproduced electron-hole pairs is referred to as an intrinsic process.

Several experimental results suggest that more than one process is responsible for the photoproduction of charged defects. Above-band-gap excitation produces two transient mid-gap electronic absorptions, at $3,900 \mathrm{~cm}^{-1}$ and $10,900 \mathrm{~cm}^{-1}$. These absorptions exhibit different lifetimes, and have different temperature and excitation wavelength dependences. The lower energy peak is associated with charged defects which give rise to transient $\mathbb{R}$ absorptions. ${ }^{14}$ The higher energy peak is indicative of a second photoprocess. With above-gap excitation, the $10,900 \mathrm{~cm}^{-1}$ absorption can be produced along with the the lower energy peak, or it can be bleached to produce the lower energy peak with low energy photoexcitation ( 9,000 to $\left.12,000 \mathrm{~cm}^{-1}\right)$. The higher energy peak has a stronger temperature dependence than the lower energy peak and, above $150 \mathrm{~K}$, is bleached in both excitation energy ranges. 23

The brief reappearance of the lower energy photoinduced absorption between 30 and $50 \mathrm{ps}$ after photoexcitation is also indicative of a second mechanism. This second peak is weaker in Baker polyacerylene, which has fewer unpaired spins. It has been suggested that the number of charged defects which can be created through this second mechanism is limited by the number of neutral defects with unpaired spins. ${ }^{25}$ This sort of mechanism, one which requires any structure other than the ground state bond-altemating polymer chain itself, is considered an exrinsic mechanism.

A low energy peak at $7.300 \mathrm{~cm}^{-1}$ in the low temperature excitation spectrum for photoinduced absorption at $3,900 \mathrm{~cm}^{-1}$ in as grown (rinsed clean, but not completely devoid of catalyst residue) polyacerylene provides direct evidence of defect mediated photoproduction of charged defects. ${ }^{27}$ This peak can be eliminated by compensation of the sample with $\mathrm{NH}_{3}$. Compensation is believed to remove the adventitious $n$-doping caused 
by residual catalyst in as grown polyacetylene. The frequency of this absorption, 7,300 $\mathrm{cm}^{-1}$, is about the same as that observed for defects induced by light chemical doping. This absorption has been assigned to positively charged solitor. . Orenstein has proposed that photoexcitation of positively charged solitons with $7,000 \mathrm{~cm}^{-1}$ or higher frequency light creates a neutral soliton and a hole. If the hole is on the same chain it can recombine with the neutral soliton to produce a charged soliton. If the hole is on a different chain it can travel as a charged polaron until it is trapped by another neutral soliton, creating a charged soliton at some delayed time. If the second neutral soliton is not next to a negatively charged impurity the positive charge will eventually hop to a soliton site where it is bound to a counter-ion. The observed transient behavior in lightly doped polyacetylene supports this proposal. Upon photoexcitation some of the absorption strength at $1390 \mathrm{~cm}^{-1}$, due to dopant induced charged defects, is temporarily lost to absorption at $1370 \mathrm{~cm}^{-1}$, where photoinduced charged defect absorption is typically observed.

Etemad ${ }^{18}$ has suggested an extrinsic mechanism for the photoproduction of charged defects in which charged defects and electrons or holes are produced by photoionization of neutral solitons. EPR data suggests that the density of neutral defects in Shirakawa polyacetylene is sufficient that their electronic absorption should be observable. Since there is no observable transition near the middle of the band gap and the $12,000 \mathrm{~cm}^{-1}$ shoulder on the absorption-edge is not affected by compensation Etemad assigned the shoulder to neutral soliton absorption. The structure on this peak has been attributed to vibrational spacings for a symmetric vibration of a neutral soliton, $920 \mathrm{~cm}^{-1}$ in $(\mathrm{CH})_{\mathrm{x}}$ and $800 \mathrm{~cm}^{-1}$ in (CD) $x$. ${ }^{28}$ The electronic state associated with this transition is pictured in the upper half of the band gap because the vibrational progression is on the high energy side of the absorption. This suggests that this transition is between a neutral soliton and the conduction band. Thus, photoexcitation near $12,000 \mathrm{~cm}^{-1}$ is expected to convert a neutral soliton to a negatively charged soliton and a hole. Again the hole is expected to travel as a charged polaron unil it is trapped by a neutral soliton, creating a charged soliton at some time delay. Etemad proposed that the $10,900 \mathrm{~cm}^{-1}$ peak which can be either photoinduced or photobleached is essentially the same neutral soliton transition.

Vardeny ${ }^{29}$ has pointed out that the high energy photoinduced absorption at 10,900 $\mathrm{cm}^{-1}$ must be due to an intrinsic mechanism because it does not saturate; it can be produced on any stretch of bond-alternating trans-polyacetylene. Similarly, the low energy photoinduced absorption at $3,900 \mathrm{~cm}^{-1}$, which does saturate at relatively low defect concentration, ${ }^{30}$ would be associated with an extrinsic process, perhaps limited by the initial concentration of neutral defects. Vardeny assigned the high energy transient absorption to neutral soliton pairs. Su had predicted that charge-conjugation symmetry 
would prevent the production of neutral soliton pairs from electron-hule pairs. However, Kivelson ${ }^{31}$ has shown that even weak electron-electron interactions can relax this symmetry constraint and allow interacting charged soliton pairs to be converted to neutral soliton pairs weakly bound to each other. Vardeny suggested that these neutral soliton pairs are metastable and can be converted back to charged solitons by collisions or photoexcitation.

The general consensus is that there are several processes, both intrinsic and extrinsic, which can contribute to the photoproduction of charged defects. Electron-hole pairs can be photogenerated on the same chain or on separate chains. Electron-hole pairs produced on the same chain relax to charged solitons (with an absorption at $3,900 \mathrm{~cm}^{-1}$ ) which can be converted to neutral soliton pairs (with an absorption at $10,900 \mathrm{~cm}^{-1}$ ). Neutral soliton pairs eventually recombine or decay to charged solitons, or can be photoconverted to charged solitons. Unpaired electrons and holes can be produced by interchain absorption or photoexcitation of existing defects. Unpaired electrons and holes become charged polarons until they are trapped by neutral solitons and become charged solitons. The charges on isolated solitons can hop to neutral solitons, near oppositely charged solitons with which they can recombine, or near charged impurities to which they can bind. Evenually through hopping and trapping all the photoinduced charges recombine. All this happens and the polymer is returned to its original state within a millisecond.

There are several problems with these explanations. The assumption that the photoinduced defects are solitons not polarons is based on Flood and Heeger's photomodulated EPR work which suggests that no unpaired spins are produced during photoexcitation. It has been argued, on the basis of defect concentration, that these results are not definitive. ${ }^{23}$ This is fortunate since the above proposed mechanisms rely heavily on the production of such spin containing defects as charged polarons and neutral solitons. The invocation of charged polarons creates another problem in finding agreement between experiment and theory. Charged polarons are predicted to have IR absorptions similar to and much stronger than those predicted for charged solitons, but no $\mathbb{R}$ activity has been linked to the charged polarons proposed in the above scenarios. Furthermore, no mention is made of the millisecond liferimes originally observed for these absorptions. 14

An additional and troubling failing of the above proposed schemes is their incompatibility with the present findings that photoinduced IR activity can be observed at extremely long times (on the hour time scale) after photolysis. The present findings also suggest that the long-lived IR active defects are either due to, or accompanied by, defects with unpaired spins. 


\section{D.1 Persistent Photoinduced Mid-Gap States}

It has been shown in Chapter III that long-lived photoinduced IR absorptions which persist for hours can be induced by excitation of a transition with an absorption onset near $5,000 \mathrm{~cm}^{-1}$ and an apparent maximum near $9,000 \mathrm{~cm}^{-1}$. The shape of this feature beyond $9,000 \mathrm{~cm}^{-1}$ could not determined with any certainty because of the optical density of the sample. The long-lived photoinduced $\mathbb{R}$ absorptions can be depleted by excitation of a transition which extends from 3,000 to $8,690 \mathrm{~cm}^{-1}$. This transition has a maximum near $4,000 \mathrm{~cm}^{-1}$, much like the near-IR absorption induced by heavy chemical doping or the transient photoinduced near-IR absorption:associated with the photoinduced $\mathbb{R}$ absorptions. It can be assumed that the $4,000 \mathrm{~cm}^{-1}$ absorption is due to the same structural defect as the observed mid-IR absorptions. It appears that this defect is destroyed or severely modified by photoexcitation near $4,000 \mathrm{~cm}^{-1}$.

There are several previously observed transitions in the band gap which could have the same origin as the high energy feature responsible for the photoproduction of $\mathbb{R}$ active defects.

\section{D.2 Defect Densities}

Blanchet 22 and Vardeny ${ }^{14}$ report maximum densities for photoinduced charged defects of $5 \times 10^{17} \mathrm{~cm}^{-3}$ and $4 \times 10^{17} \mathrm{~cm}^{-3}$, respectively. These defects were observed to live for a few milliseconds in 0.1 to $0.5 \mu$ thick samples. The densities were calculated using the per defect absorptivity at the peak maximum of the $4,000 \mathrm{~cm}^{-1}$ absorption, $\alpha=4$ $x 10^{-16} \mathrm{~cm}^{2}$, derived from the optical absorption of lightly electrochemically doped polyacetylene. ${ }^{7}$ The ratios of the relative peak heights for the $1,370 \mathrm{~cm}^{-1}$ and $3,900 \mathrm{~cm}^{-1}$ absorbances derived from Blanchet and Vardeny's data are 0.7 to 0.47 , respectively. This corresponds to per defect absorptivities at $1370 \mathrm{~cm}^{-1}$ of $2 \times 10^{-16} \mathrm{~cm}^{2}$ and $3 \times 10^{-16} \mathrm{~cm}^{2}$. The theoretical values for the per defect absorptivity at $1370 \mathrm{~cm}^{-1}$ for solitons and polarons are $8 \times 10^{-17}$ and $8 \times 10^{-16}$ respectively. ${ }^{1}$ From these absorptivities defect densities of $3 \times$ $10^{16} \mathrm{~cm}^{-3}$ and $2 \times 10^{16} \mathrm{~cm}^{-3}$ can be calculated for the persistent photoinduced defects introduced in Chapter III.

Flood and Heeger ${ }^{32}$ report a density of $10^{19} \mathrm{~cm}^{-3}$ spin defects in both photoexcited polyacetylene and the same sample up to a tenth of a second after photoexcitation. The accuracy with which they controlled the temperature of their sample allowed for $0.1 \%$ variations in integrated signal intensity at $10 \mathrm{~K}$, and $0.01 \%$ variations at $300 \mathrm{~K}$. Thus, they were able to say that photolysis with an $\mathrm{Ar}^{+}$ion laser at $20-100 \mathrm{mWcm}^{-2}$ produced fewer than $10^{16} \mathrm{~cm}^{-3}$ at $10 \mathrm{~K}$ and $10^{15} \mathrm{~cm}^{-3}$ at $300 \mathrm{~K}$ spin containing defects with lifetimes $<100$ ms. The experimental conditions used were intended to mimic those under which $5 \times 10^{17}$ 
$\mathrm{cm}^{3} \mathrm{IR}$ active defects with apparent millisecond lifetimes have been observed. Flood and Heeger conclude that the transient photoinduced defects with apparent millisecond lifetimes which are observed in the $\mathbb{R}$ are spinless, i.e. charged solitons.

The EPR data in Chapter III reports a 5\% change in the density of spin defects under conditions which produce and destroy persistent IR active defects. The spin defect densities reported for trans-polyacetylene typically range from $6 \times 10^{18}$ to $2 \times 10^{19.33}$ Five percent of this is $3 \times 10^{17}$ to $1 \times 10^{18}$ persistent photoinduced spin defects per $\mathrm{cm}^{3}, 10$ to 50 imes the calculated density of photoinduced persistent IR active defects.

\section{D.3 Lifetime Discrepancies}

With the observation of persistent photoinduced IR active defects, Chapter III, there are now three timescales on which such defects have been observed. The existing proposed mechanisms, which only attempt to explain photoinduced defects on one of these timescales, are already plagued with problems. The introduction of a third timescale might be expected to make this bad situation impossible. Nevertheless, it is possible that these new findings will allow the use of a more simple mechanism. It is suspected that the persistent and millisecond lifetime defects have the same origin. The difference in the observed lifetimes is proposed to be caused by differences in the experimental conditions.

The difference observed in the IR spectra of a polyacetylene sample after photolysis with $\mathrm{Ge}\left(v<5,500 \mathrm{~cm}^{-1}\right)$ - and $\mathrm{CG}+\mathrm{H}_{2} \mathrm{O}\left(7,600-16,500 \mathrm{~cm}^{-1}\right)$-filtered light, Chapter III, are seen only if the spectrometer beam is filtered, blocking $v>2,400 \mathrm{~cm}^{-1}$. It is assumed that a steady-state $\mathbb{R}$ activity level characteristic of probe beam photolysis was established before the IR activity levels induced by photolysis with $\mathrm{Ge}\left(v<5,500 \mathrm{~cm}^{-1}\right)$ or $\mathrm{CG}+\mathrm{H}_{2} \mathrm{O}\left(7,600-16,500 \mathrm{~cm}^{-1}\right)$-filtered light could be recorded. The spectra were the average of 200 scans taken within the first 2 minutes that the sample was in the IR beam. Since one tenth of the observed signal could have been detected easily, the probe beam photolysis steady-state must have been established within 24 seconds for a $20 \mu$ thick sample.

The millisecond lifetime for photoinduced defects was determined using a $0.1 \mu$ thick sample. ${ }^{14}$ The number of persistent defects similar to those observed in this work, which could be produced in such a sample, would have been depleted by the IBM IR/44 spectrometer beam within $120 \mathrm{~ms}$. This is an upper limit on the lifetime of these defects in the spectrometer beam path; there is no reason to believe it is not considerably shorter. The millisecond lifetime for transient photoinduced $\mathbb{R}$ active defects was determined by probing with a globar monochrometer set-up of unreported intensity. ${ }^{14}$ The IR activity for which 
the millisecond lifetime was reported may have been caused by defects which would have persisted on a much longer timescale if protected from the probe beam.

This possibility seems a little less likely when the flux required to deplete the photoinduced $\mathbb{R}$ activity is calculated. According to the kinetic data in Chapter III, $1.5 \mathrm{x}$ $10^{24} 4,000 \mathrm{~cm}^{-1}$ photons are required to deplete half of the maximum photoinduced $\mathrm{IR}$ activity. Vardeny's chopping frequency data shows that the above-gap photoinduced $\mathbb{R}$ absorptions are reduced to half intensity in $1 \mathrm{~ms}$. A $1000 \mathrm{~K}$ globar emits only $4 \times 10^{19}$ photons per second, $4 \times 10^{16}$ per ms, between 2,500 and $5,500 \mathrm{~cm}^{-1}$. This calculation suggests that photolysis near $4,000 \mathrm{~cm}^{-1}$ caused by the unfiltered probe beam is insufficient to account for the rapid loss of IR activity observed in the unfiltered probe beam. There is something wrong with either the kinetic data, this calculation or the assertion that photolysis is the mechanism by which the probe beam destroys the photoinduced IR activity.

It is nevertheless an experimental fact that the lifetime of photoinduced IR defects observed in Chapter III is greatly reduced by the unfiltered probe beam. This effect is pronounced enough to suggest that the $\mathbb{R}$ activity attributed to defects with millisecond lifetimes is actually due to long-lived defects which are rapidly destroyed by the probe beam. This destructive probe beam was not included when Flood and Heeger attempted to duplicate the experiment with which the millisecond lifetime was determined in the EPR.

The previously reported study on photoinduced defects with picosecond lifetimes also suggest that there are no photoinduced defects with millisecond lifetimes. 25 This experiment was run at $10 \mathrm{~Hz}$, that is at $100 \mathrm{~ms}$ intervals, allowing time for the defects with millisecond lifetimes to disappear before each photoexcitation. A photoinduced absorption at $4,000 \mathrm{~cm}^{-1}$ was observed for the first $10 \mathrm{ps}$, and at $10 \mathrm{~K}$, again 30 to $50 \mathrm{ps}$ after photolysis. All of the observed photoinduced absorption decayed within $1000 \mathrm{ps}, 10^{-6} \mathrm{~ms}$. If photoinduced defects with millisecond lifetimes were produced in this experiment the decay of their absorption at $4,000 \mathrm{~cm}^{-1}$ should have been observed between $1000 \mathrm{ps}$ and $10 \mathrm{~ms}$. The picosecond, picojoule IR pulses used in this experiment to monitor the 4,000 $\mathrm{cm}-1$ absorption could not have had as profound an effect on the system proposed for IR spectrometer beams. Thus defects which might be depleted in a few milliseconds in a conventional IR beam would not decay perceptibly during the $10 \mathrm{~ms}$ between repetitions. These defects could have remained IR active throughout the experiment and been recorded as part of the background. 


\section{D.4 EPR Discrepancies}

The EPR results reported in Chapter III are not the same as those reported by Flood and Heeger, 32 but they are not incompatible. There is no reason to expect transient IR active defects produced by above-gap photoexcitation to have the same origin as persistent IR active defects produced by mid-gap photoexcitation. However, the similarities in the spectra of these defects and the possibility that the shorter lifetime previously observed is an artifact of the experimental conditions are cause for concern. The major difference in the experimental conditions under which millisecond lifetimes were observed for $\mathbb{R}$ active photoinduced defects and those used for Flood and Heeger's EPR experiment is that there was no potentially destructive IR probe beam in the latter. It is possible, that as suggested for the picosecond experiment, the long-lived defects did not decay significantly between repetitions and were recorded as part of the background. In this case the maximum induced spin densities reported should be correlated with the defects observed on the picosecond timescale, not those with longer lifetimes. The density of the photoinduced defects with absorptions in the near $\mathbb{R}$ and picosecond lifetimes is not reported.

In the EPR experiment presented in Chapter III the spin containing defects created with $\mathrm{CG}+\mathrm{H}_{2} \mathrm{O}\left(7,600-16,500 \mathrm{~cm}^{-1}\right)$-filtered light were deliberately destroyed by subsequent photolysis with $\mathrm{Ge}\left(\mathrm{v}<5,500 \mathrm{~cm}^{-1}\right)$-filtered light. More spin defects were detected in the sample after photolysis with $\mathrm{CG}+\mathrm{H}_{2} \mathrm{O}\left(7,600-16,500 \mathrm{~cm}^{-1}\right)$-filtered light than after photolysis with $\mathrm{Ge}\left(v<5,500 \mathrm{~cm}^{-1}\right)$-filtered light. This indicates that spin containing defects are created and depleted by the same photolysis frequencies as IR active defects. The samples used in the EPR experiment may have been sufficiently optically dense at the wavelengths used for photolysis, because of the manner in which the sample. was stuffed into the EPR tube, that regions of the polymer may not have been photolysed. This would have systematically lower the number of photoinduced defects observed in the EPR experiment relative to those produced in the IR experiment. The rough defect density calculations above suggest that 10 to 50 times more defects were observed in the EPR experiment than in the IR experiment.

Combined, the IR and EPR experimental results suggest either that both charged and spin containing defects are produced by photolysis with $\mathrm{CG}+\mathrm{H}_{2} \mathrm{O}(7,600-16,500$ $\mathrm{cm}^{-1}$ )-filtered light or that the photoinduced defects have both charge and spin. These data cerrainly rule out the possibility that long-lived IR activity is due to charged solitons formed by the trapping of introduced charges by neutral solitons, as suggested by Orenstein. 27 


\section{D.5 Asymmetric Depletion}

The dopant induced defect absorption at $1390 \mathrm{~cm}^{-1}$ has been observed to shift 18 $\mathrm{cm}^{-1}$ to lower frequencies when the dopant concentration is increased from $0.03 \%$ to 10\%.2 The near-IR absorption near $4,000 \mathrm{~cm}^{-1}$ also shifts, more dramatically $7,000 \mathrm{~cm}^{-1}$ to $4,000 \mathrm{~cm}^{-1}$, to lower energy with increasing dopant concentration. 6 The asymmetric photodepletion of the persistent photoinduced IR absorption at $1370 \mathrm{~cm}^{-1}$ observed during photolysis of the low energy side of the $4,000 \mathrm{~cm}^{-1}$ electronic absorption is consistent with these trends. The defects in more dense regions have lower energy absorption maxima. They absorb low energy photolysis light more strongly and are destroyed more rapidly by low energy photolysis than more isolated defects. This is illustrated by the preferential loss of the low frequency side of the IR peak at $1370 \mathrm{~cm}^{-1}$.

The $1370 \mathrm{~cm}^{-1}$ photoinduced IR absorption peak also decays asymmetrically in the dark. The low frequency side of the absorption, due to less isolated defects, disappears first. Thus, density dependant mechanisms, such as recombination and short range charge hopping, should be considered as probable causes for the decay observed in the dark. A density dependent rate is consistent with the non-exponential form of the dark decay curve (Figure III-3). The fact that both low frequency photodepletion and the dark decay are asymmetric might alternately suggest that low energy probe beam or black body photolysis is responsible for the dark decay. However, both these possibilities have been discounted. Low energy probe beam photolysis is not believed to be responsible for the dark decay because no increase was observed in the rate of decay during photolysis at frequencies passed by the probe beam filter $(v<2,400)$. The flux required to obtain the rate of decay observed in the photoinduced $\mathbb{R}$ activity of samples kept in the dark, calculated from the kinetic data in Chapter III, is at least six orders of magnitude greater than that emitted from a room temperature black body. This calculation suggests that the observed dark decay cannot be accounted for by black body irradiation from the room temperature surroundings. Since some question has been raised about the validity of this sort of calculation (Chapter IV section D.3), it should be pointed out that decay mechanisms which involve photolysis cannot account for the observed dependence of the rate of decay on isomer content.

The density of photoinduced IR active defects observed during photolysis ${ }^{22}$ is ten times that detected a few minutes after photolysis (this work). This difference in defect density is reflected in the location of the $1370 \mathrm{~cm}^{-1}$ peak. When observed during photolysis the $1370 \mathrm{~cm}^{-1}$ peak is iucated at $1365 \mathrm{~cm}^{-1}, 34$ one minute after photolysis it has shifted to $1369 \mathrm{~cm}^{-1}$. Again the defects in more dense regions are the first to disappear. The high density rate of decay inferred from the comparison of these experiments is much 
faster than the decay rate observed at lower densities, supporting the theory that the dark decay is caused by a density dependent process.

The maximum obtainable signal (Table III-3) and the steady-state signal 22 decrease with increasing temperature. The dark decay rate for the fraction of the maximum obtainable signal is not affected by temperature (Table III-4). Thus, the density dependence of the dark decay rate is different at different temperatures. This inuicates that the mechanism responsible for the decay is facilitated by increasing temperature. The maximum obtainable signal decreases gradually with increasing temperature between $10 \mathrm{~K}$ and $125 \mathrm{~K}$. The temperature dependence of the steady-state signal has been observed at higher temperatures. ${ }^{22}$ A sudden increase in the rate of intensity loss with increasing temperature is observed near $150 \mathrm{~K}$.

Two mechanisms are suggested for the cause of the dark decay, recombination and short range charge hopping. The recombination rate should be dependent on the number of defect collisions. The number of collisions is dependent on the defect density and mobility of the defects. The effectiveness of short range charge hopping should depend on the distance the charge can hop and the distance between defects. Either one or both of these could increase with increasing temperature.

The temperature dependence of the maximum obtainable signal reflects the temperature dependence of either the defeet mobility or the charge hopping range. For any given defect density a faster rate of decay is expected for more mobile defects or greater hopping ranges. The temperature dependence of the maximum obtainable signal and steady-state signal indicate that the defects become more mobile or the charge hopping range increases as the temperature increases. The suggested increase in the defect mobility or charge hopping range with increasing temperature is gradual between 10 and $125 \mathrm{~K}$ and increases sharply above $150 \mathrm{~K}$.

The dependence of the rate of the dark decay on isomer content suggests that mobility limits the rate of decay. Defect mobility along the chain is expected to be significantly hampered by cis segments, through which the defects cannot travel. The observed decrease in the rate of dark decay with decreasing trans fraction is consistent with this expectation. As the trans fraction increases, the number of sites capable of supporting non-bond altemation defects increases. The dominant factor in the dependence of the maximum obtainable signal on the trans fraction (Table III-3) is the increase in the number of sites capable of supporting defects. The increase in the ease with which the defects can destroy each other, with increasing trans content, moderates this dependence. 


\section{E.1 Photoproduction of Persistent Defects with Both Charge and Spin}

The experimental data presented in Chapter III indicate a greater concentration of both charged (IR active) and spin containing defects in trans-polyacetylene after photolysis with $\mathrm{CG}+\mathrm{H}_{2} \mathrm{O}\left(7,600-16,500 \mathrm{~cm}^{-1}\right)$-filtered light than after photolysis with $\mathrm{Ge}(v<$ $5,500 \mathrm{~cm}^{-1}$ )-filtered light. The approximate density of additional spin containing defects, calculated in section IV.D.2, is $\mathbf{1 0}$ - 50 times greater than that calculated for IR active def _ets. The difference in these densities could be due in part to the unknown difference in the isomer content of the samples used and/or the difference in the temperatures at which the EPR and IR experiments were conducted, $4.2 \mathrm{~K}$ and $10 \mathrm{~K}$ respectively. The calculated number of spin containing defects and number of charged defects are similar given the difference in the experimental setups and the accuracy of the calculations. It is quite possible that under the identical experimental conditions the number of spin containing defects and charged defects detected would be the same. Assuming that this is the case, the formation of a single type of defect, charged polarons, can account for both the additional spins and increased IR activity.

Charged polarons can be produced by mid-gap photolysis via a sequential two photon process, using neutral defects as a stepping stone. Two mechanisms for the photoproduction of charged polarons are presented (illustrated in Figures IV-5 and IV-6). The minimum photon energy required for the photoproduction of charged polarons by the first of these mechanisms is the same as that observed in this work, $4,000 \mathrm{~cm}^{-1}$, lower than that previously observed for the photoproduction of charged defects. The second proposed mechanism involves transitions known to have stronger absorption cross sections than one of the transitions used in the first mechanism. This mechanism predicts an excitation spectrum similar to that previously observed for the photoproduction of transient IR active defects and the photoconductive response curve.

The electronic state associated with a neutral soliton is located $11,000 \mathrm{~cm}^{-1}$ above the valence band. An electron can be promoted from the valence band to a neutral soliton by an $11,000 \mathrm{~cm}^{-1}$ photon. The transition from a neutral soliton to the conduction band, at 4,700 $\mathrm{cm}^{-1}$ with an onset near $2,500 \mathrm{~cm}^{-1}$, has not been observed directly. When this transition is photoexcited a positively charged soliton and a conduction band electron are produced (A1). The conduction band electron is accommodated by the polymer through the formation of a charged polaron. An absorption at $7,300 \mathrm{~cm}^{-1}$, with an onset at $5,100 \mathrm{~cm}^{-1}$, has been assigned to positively charged solitons. When a positive soliton absorbs light above $5,100 \mathrm{~cm}^{-1}$ (A2) an electron is promoted from the valence band to the defect. This transition returns the soliton to its original oxidation state and leaves a hole in the valence band, which prompts the formation of a positively charged polaron. 


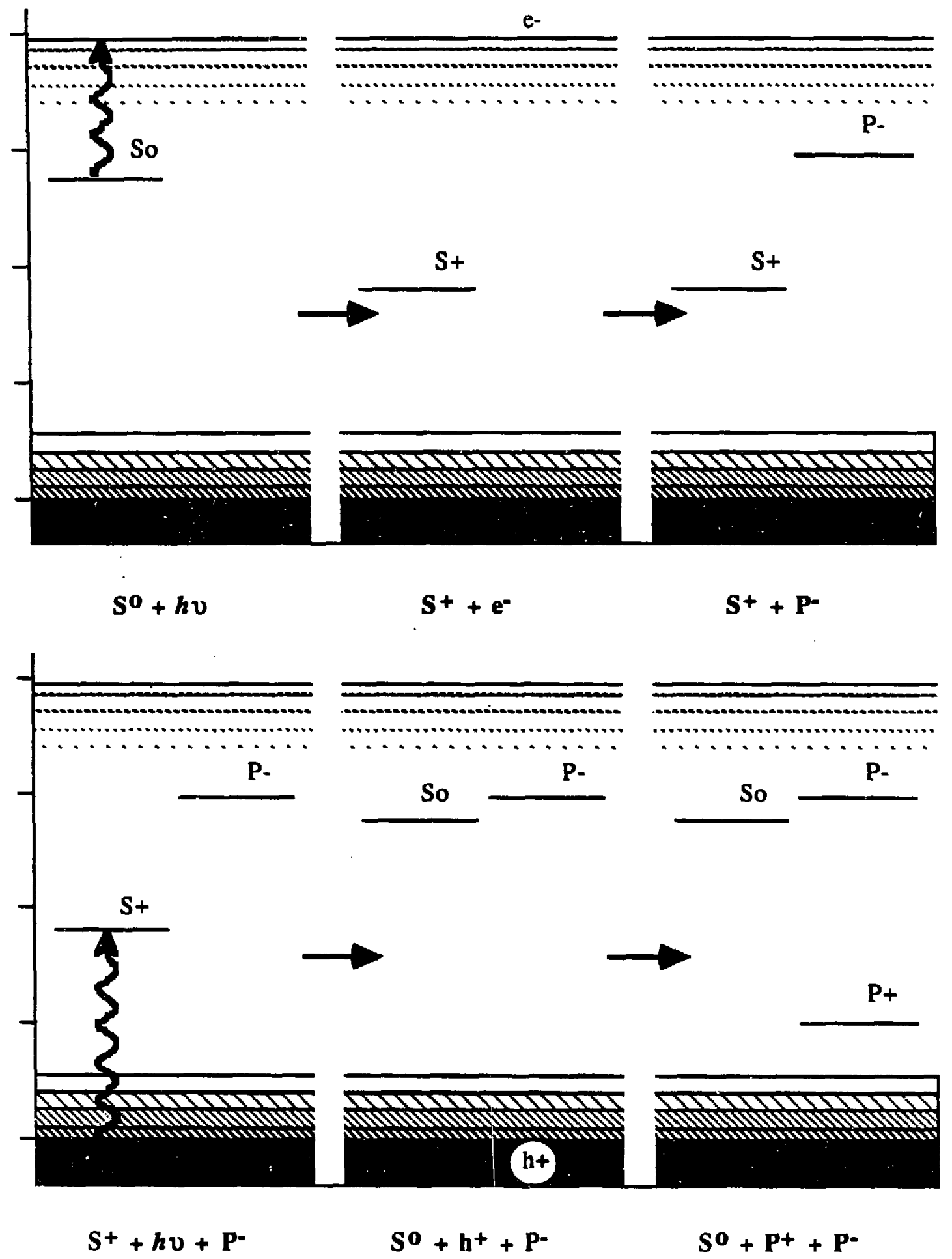

Figure IV-5 Cycle A

Two photon process producing a positively and a negatively charged polaron 

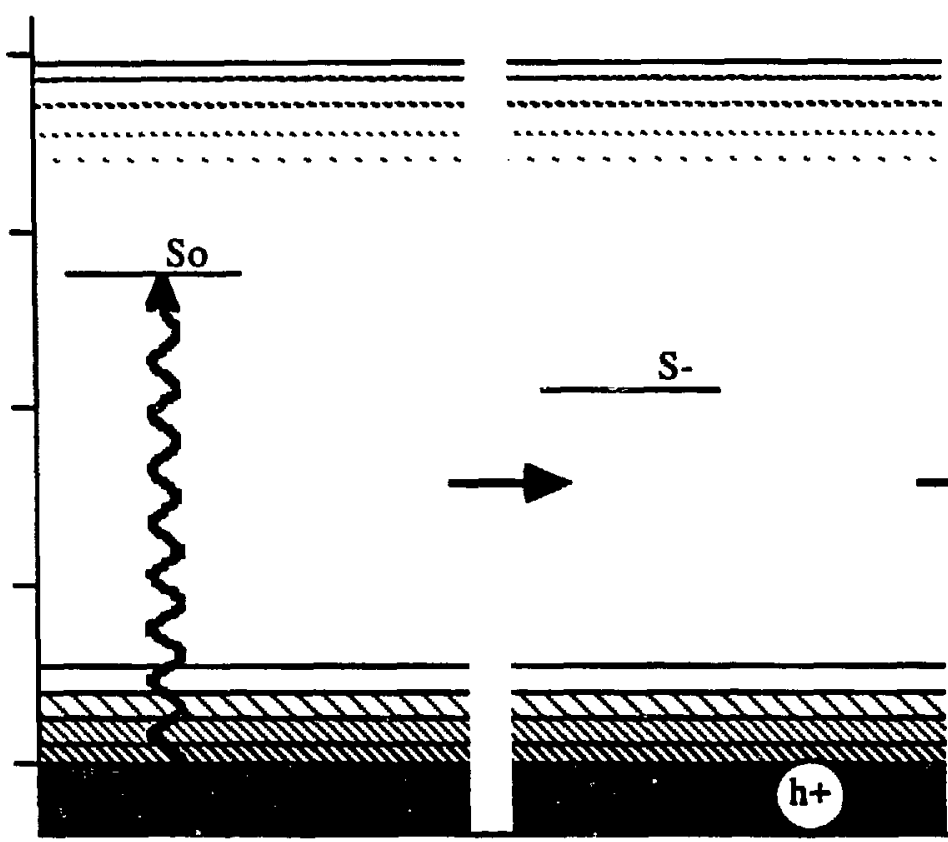

S-

$$
S^{0}+h v
$$

$S^{-}+h^{+}$

$\mathbf{S}^{-}+\mathbf{P}^{+}$
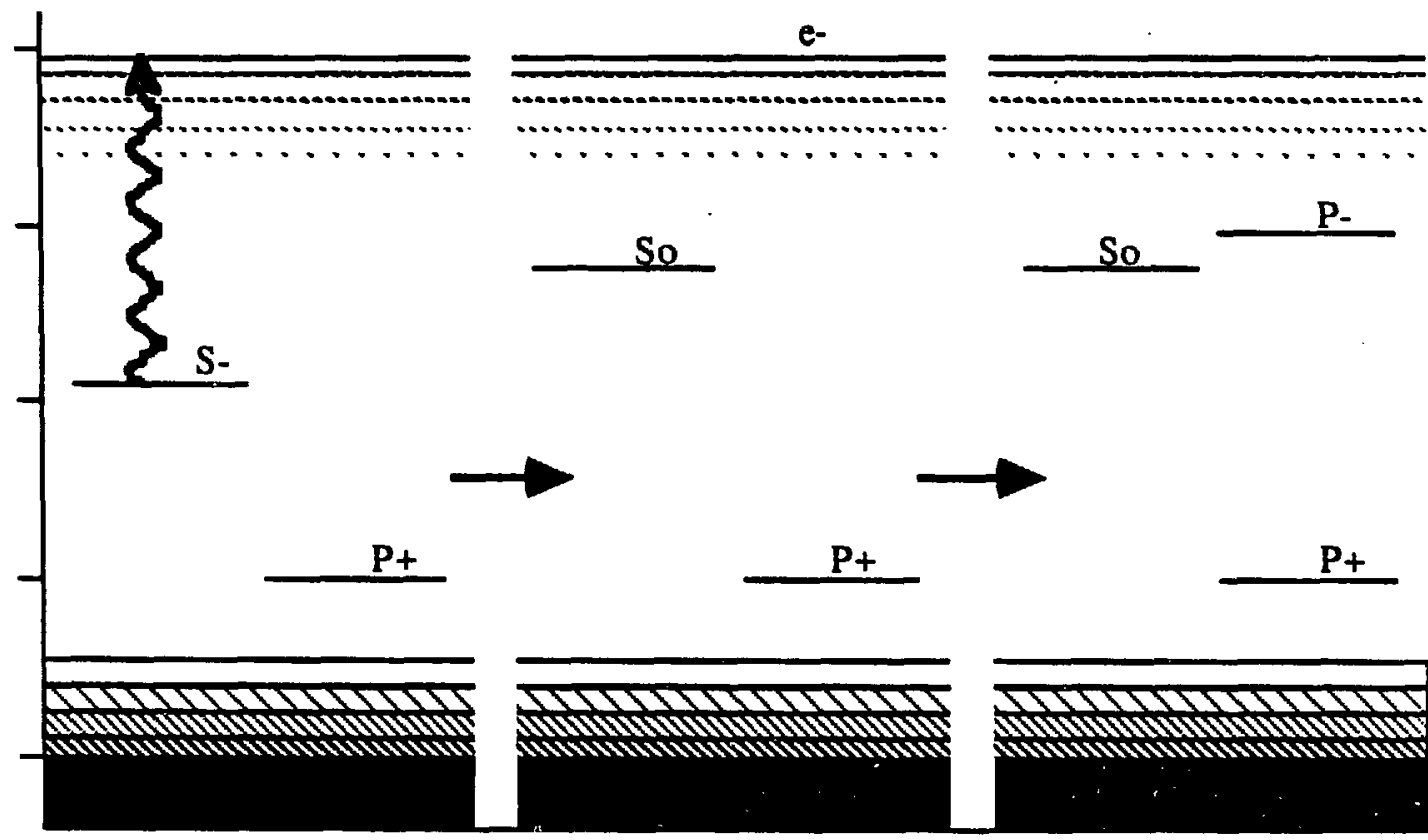

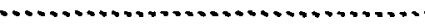

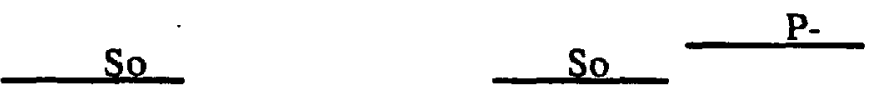

$\mathrm{S}^{\bullet}+\boldsymbol{h} v+\mathrm{P}+$

$\mathrm{SO}^{\mathrm{O}}+\mathrm{e}^{-}+\mathrm{P}+$

$\mathrm{S}^{\mathbf{0}}+\mathbf{P}^{-}+\mathbf{P}^{+}$

Figure IV-6 Cycle B

Two photon process producing a positively and a negatively charged polaron 


$$
S^{0}+h v \rightarrow S^{+}+e^{-}(C B) \rightarrow S^{+}+P^{-}
$$

$$
\mathrm{S}^{+}+h v \rightarrow \mathrm{S}^{\mathrm{O}}+\mathrm{h}^{+}(\mathrm{VB}) \rightarrow \mathrm{S}^{\mathrm{O}}+\mathrm{P}^{+}
$$

A second mechanism provides an alternate route for the formation of charged spincontaining defects with mid-gap photolysis, also catalyzed by neutral solitons. An electron can be promoted from the valence band to a neutral soliton, coriverting it to a negatively charged so" : in and leaving a hole in the valence band. The polymer accommodates the hole by fo ming a positively charged polaron. The negatively charged soliton can be converted back to a neutral soliton by the promotion of one of its electrons to the conduction band. Again, the polymer accommodates the conduction band electron with the formation of a polaron.

$$
\begin{aligned}
& \mathrm{S}^{0}+h v \rightarrow S^{-}+h^{+}(\mathrm{VB}) \rightarrow \mathrm{S}^{-}+\mathrm{P}^{+} \\
& \mathrm{S}^{-}+h v \rightarrow \mathrm{S}^{0}+\mathrm{e}^{-}(\mathrm{CB}) \rightarrow \mathrm{S}^{0}+\mathrm{P}^{-}
\end{aligned}
$$

The transitions involved in cycle (B) have absorption maxima at 11,000 and 4,000 $\mathrm{cm}^{-1}$. The minimum energy photons required for each step are $8,800 \mathrm{~cm}^{-1}$ for step (B1) and $1,800 \mathrm{~cm}^{-1}$ for step (B2). The higher energy photon must be present to complete the entire cycle. The excitation spectrum for this cycle, limited by step (B1), is the same as that observed for photoconductivity and photoproduction of transient defects. Cycle (A) involves the transition from neutral defects to the conduction band, a transition which has not been directly observed. Steps (A1) and (A2) require $2,500 \mathrm{~cm}^{-1}$ and $5,100 \mathrm{~cm}^{-1}$ photons, respectively. Thus, as observed in this work, persistent photoinduced defects can be produced with $5,100 \mathrm{~cm}^{-1}$ light. The observation of charged defects photoinduced by $v$ $>5,000 \mathrm{~cm}^{-1}$ provides indirect evidence of this transition. Because one of the transitions involved, the neutral defect to conduction band transition, is unusually weak cycle (A) would have a lower quantum yield than cycle (B). The observation of a greater rate of production at higher energy photolysis was probably moderated by experimental difficulties with the optical density of the sample.

Because these are catalytic processes, the maximum number of stable defects produced by either of the above mechanisms is not limited by the number of neutral defects. The persistent and steady-state 22 photoinduced signals "saturate" at a low defect 
density which is imposed by the density dependent decay of these defects. However, for catalytic processes the rate of defect photoproduction does depend on the neutral defect concentration. The kinetics for catalytic processes, like those observed for the photoproduction of persistent IR active defects (Chapter III), cannot be fit with a first order rate equation. Unfortunately the data for the photoproduction of persistent IR active defects is insufficient (too few points) and too inaccurate (because of the samples optical density) to warrant attempting to fit it to a more appropriate model. The information obtained from the kinetic modeling in Chapter III, that the onset for the transition responsible for photoproduction of $\mathbb{R}$ active defects is near $5,000 \mathrm{~cm}^{-1}$, and that its absorption increases to higher frequency at least as far as $9,000 \mathrm{~cm}^{-1}$, could also be obtained by simple inspection.

\section{E.2 Photodepletion of Presistent Defects}

Some fraction of the electrons and holes generated in cycles (A) and (B) find each other and combine before the charge separation is stabilized by the formation of oppositely charged defects. Likewise, when electrons are promoted from the valence band to cationic defects and from anionic defects to the conduction band, some of the electrons and holes produced can combine. Electrons and holes are more mobile, and possibly more inclined to combine, than oppositely charged bond-alternation defects. Thus, photoexcitation of these transitions opens a new route for the depletion of the photoinduced $\mathbb{R}$ activity. The extent to which the decay rate is enhanced should be proportional to the absorption intensities of the defect to band transitions. As discussed in Section IV-D.5, at low defect concentrations, the energy level of each bond-alternation defect is effected by the separation between defects. The statistical variation in the separation between defects creates a distribution of populations with distinct absorption spectra. These distinct absorption spectra lead to different rates of photodepletion, at any given photolysis frequency, for the various populations. As observed in Chapter III, this leads to very complicated kinetic behavior.

\section{E.3 New Assignments For Mid-Gap States}

The previous assignments for the mid-gap states at $11,000 \mathrm{~cm}^{-1}$ and $7,300 \mathrm{~cm}^{-1}$ are consistent with and essential to the above scenario. The $11,000 \mathrm{~cm}^{-1}$ peak is due to the promotion of an electron from the valence band to a neutral soliton. The $7,300 \mathrm{~cm}^{-1}$ peak is due to both the promotion of valence band electrons to positively charged solitons and the promotion of electrons from negatively charged solitons (at $8,400 \mathrm{~cm}^{-1}$ above the valence band) to the conduction band. The assignment of the photoinduced absorption at $4,000 \mathrm{~cm}^{-1}$ to charged solitons is not compatible with the present experimental results or 
the above scenario, based on those results. The work presented in this thesis suggests that the photoinduced $4,000 \mathrm{~cm}^{-1}$ absorption and associated $\mathrm{IR}$ absorptions are due to charged polarons, i.e. defects without bond alternation, extending over an even number of carbons, and possessing a charge.

It is not clear that the reassignment of the photoinduced $4,000 \mathrm{~cm}^{-1}$ and $\mathrm{R}$ absorptions has any bearing on the assignment of similar absorptions induced by heavy doping. The IR spectra of defects produced by chemical doping and photolysis differ significantly in the frequency of the broad absorption at $832 \mathrm{~cm}^{-1}$ and $532 \mathrm{~cm}^{-1}$, respectively. The isotope shifts observed for these absorptions are also significantly dissimilar, 1.24 and 1.05. In the past, the difference between the frequencies of the low energy IR absorptions for dopant induced and photoinduced defects has been attributed to the difference in the pinning potentials caused by the counter-ions and oppositely defects. The difference in isotope shifts has not been addressed. Chemically induced and photoinduced defects are in very different environments; the similarity in the electronic absorptions could be partially coincidental. The differences in their IR spectra are sufficient to warrant hesitation about attributing both to the same origin.

\section{F Persistent Photoinduced Mid-Gap Defects}

Mid-gap photolysis of polyacetylene, with $v>5,000 \mathrm{~cm}^{-1}$, has been observed to produce persistent $\mathbf{R}$ absorptions. These absorptions are similar to the transient IR absorptions previously observed during photoexcitation $v>9,000 \mathrm{~cm}^{-1} .35$ The fact that the persistent IR absorptions can be observed only if the probe beam is filtered, blocking $v$ $>2,400 \mathrm{~cm}^{-1}$, suggests that the previously observed transient absorptions may in fact be persistent absorptions, destroyed in a few milliseconds by the probe beam. Like the transient IR absorptions the persistent IR absorptions are attributed to charged bondalternation defects in polyacetylene.

$\mathrm{Su}^{26}$ had predicted that the above-gap photolysis of polyacetylene would result in charged bond-altemation defects; solitons for inter-chain excitation and polarons for intrachain excitation. The observed onset for the production of transient photoinduced defects $\left(v>9,000 \mathrm{~cm}^{-1}\right)$ is well below the onset of the inter-band absorption $\left(v>11,300 \mathrm{~cm}^{-1}\right) .16$ Alternate mechanisms were needed to explain the production of transient IR active defects since they could be produced by below-gap photolysis. The observed onset for the production of persistent photoinduced defects $\left(v>5,000 \mathrm{~cm}^{-1}\right)$ is lower still. Mechanisms have been proposed for the production of transient photoinduced defects. ${ }^{16,31,27}$ The additional mechanisms proposed in this work (Chapter IV-E.1) suggest that the low energy onset observed for the photoproduction of persistent IR active defects $\left(v>5,000 \mathrm{~cm}^{-1}\right)$ is 
indirect evidence for an oprical transition promoting an electron from a neutral defect to the conduction band. No observation of this transition has been reported previously.

Orenstein ${ }^{27}$ proposed that mid-gap photolysis could promote an electron from a neutral soliton to the conduction band or from the valence band to a neutral soliton. This would result in two charged defects, a charged defect from the relaxation of the photoinduced charge and a charged soliton from the removal of an electron from or addition of an electron to the neutral soliton. If the photoinduced charge were to relax to a charged polaron this process would have no effeci on the number of overall spins. The photoinduced charge was expected to relax to a charged soliton. This would result in a net loss of unpaired spins. Flood and Heeger have observed no change in the number of unpaired spins under conditions which produce transient IR active photoinduced defects. 32 This mechanism was proposed to explain the production of photoinduced transient defects. It should also be considered as a possible mechanism for the photoproduction of persistent defects, whether the transient and persistent defects are the same or not. This mechanism explains the saturation observed for the photoinduced IR signal22 since the number of possible IR active defects is limited by the number of neutral defects in the sample. Such a process is considered an extrinsic process.

The qualitative EPR experiments reported in Chapter III-F, suggest that an increase in the number of unpaired spins accompanies the production of $\mathbf{R}$ active defects. Thus the photointerconversion mechanism discussed above cannot apply to the photoproduction of persistent defects. The mechanism proposed herein is not extrinsic in the same sense as the proposed photointerconversion mechanism. The observed "saturation" limit is believed to be a steady-state value determined by the relative rates of a defect limited production process and a defect density dependent decay. The dark decay of the persistent photoinduced defects appears to be density dependent. The number of collisions or charge hops leading to defect recombination should increase with defect density. A faster rate of decay is observed in samples with a higher trans isomer fraction. Since greater defect mobility is expected in the trans isomer, the dark decay is atrributed to the collisional recombination. Thus the temperature dependence of the maximum observed signal should be indicative of the temperature dependence of the defect mobility.

Two cyclic processes have been proposed for the photoproduction of IR active defects in polyacerylene. Both of these proposed two photon cycles depend on the presence of neutral solitons. The first photon promotes an electron either from a neutral soliton to the conduction band or from the valence band to a neutral soliton. A second photon then converts the resultant charged soliton back to a neutral soliton by returning or removing an electron from the valence band or to the conduction band. The net result of 
either cycle is the production of an electron-hole pair. The polymer is expected to accommodate this electron-hole pair by the formation of two charged bond-alternation defects.

Because the EPR experiments in Chapter III-F suggest that an increase in the number of unpaired spins accompanies the observed increase in IR activity, the proposed cyclic mechanisms have been discussed in terms of polaron formation. Further work is required to substantiate this assignment because of the problems experienced with temperature stability during this experiment. Further, the temperature and frequency dependence of the change in the EPR signal must be determined to established a one to one correspondence between photoinduced charged defects and unpaired spins. Should future EPR work discount that reported herein, essentially the same mechanisms could be used to explain the photoproduction of charged solitons. The only difference would be that photogenerated electrons and holes would result in the formation of charged solitons, not charged polarons.

The rate at which the persistent photoinduced IR absorptions decay has been observed to increase during photoexcitation of an electronic transition near $4,000 \mathrm{~cm}^{-1}$. A similar electronic absorption has been associated with the transient photoinduced IR active defects. 22 The use of this electronic absorption to monitor the transient photoinduced may be responsible for the lifetimes previously observed for photoinduced defects. It is possible that the precautions taken to protect photoinduced defects from electronic excitation in the experiments described in Chapter III-E have allowed for the first accurate measurement of the actual lifetimes for photoinduced defects. If the transient photoinduced defects previously reported do in fact have intrinsic millisecond lifetimes, this work is the first reported observation of persistent photoinduced defects in polyacetylene. 


\section{References}

1 E. J. Mele, J. C. Hicks Phys. Rev. B32(4), 2703 (1985)

2 J. F. Rabolt, T. C. Clark, G. B. Street, J. Chem Phys. 71(11), 4614 (1979)

3 Y.-W. Park, A. J. Heeger, M. A. Druy, A. G. MacDiarmid, J. Phys. Chem. 73(2), 946 (1980)

4 A. J. Epstein, H. Rommelmann, M. Abkowitz, H. W. Gibson, Mol. Cryst. Liq. Cryst. 77, 81.(1981)

5 M. Audenaert, G. Gusman, R. Deltour, Phys. Rev. B24(12) 7380 (1981)

6 H. Fujimoto, J. Tanaka, M. Tanaka, Bull. Chem. Soc. Jpn. 56, 671 (1983)

7 A. Feldblum, J. H. Kaufman, S. Etemad, A.J. Heeger, T. C. Chung, A. G. MacDiarmid, Phys. Rev. B26(2),815 (1982)

8 A. G. MacDiarmid, A. J. Heeger, Synth. Metals 1, 101 (1979/1980)

9 T. C. Clark, G. B. Street, Synth. Metals 1, 119 (1979/1980)

10 T. C. Clark, R. H. Geiss, W. D. Gill, P. M. Grant, H. Morawitz, G. B. Street, Synth. Metals, 1, 21(1979/1980)

11 K. Watanabe, T. Nakayama, J. Mottl. J. Quant: Spectroscopy and Energy Transfer, 2, 369 (1962)

12 W. E. Wentworth, W. Ristan, J. Phys. Chem.73, 2126 (1969)

13 R. J. Cohen, A. J. Glick, Phys. Rev. B36(50), 2907 (1987)

14 Z. Vardeny, J. Orenstein, G. L. Baker, Phys. Rev. Lett. 50(25), 2032 (1983)

15 H. Shirakawa, S. Ikeda, Polymer J. 2(2), 231 (1971)

16 S. Etemad, A. J. Heeger, A.G. MacDiarmid, Ann. Rev. Phy. Chem., 33, 443 (1982)

17 H. Eckhardt, R. R. Chance, Phys. Chem. 79(11), 5698 (1983)

18 S. Etemad, G. L. Baker, C. B. Roxlo, B. R. Weinberger, J. Orenstein, Mol. Cryst. Liq. Cryst. 117, 275 (1985)

19 N. Suzuki, M. Ozaki, S. Etemad, A. J. Heeger, A. G. Macdiarmid, Phys. Rev. Lett. 45, 12.09 (1980)

20 G. L. Baker, J. A. Shelbume III, F. S. Bates, J. Am. Chem. Soc. 108, 7377 (1986)

21 B. R. Weinberger, Phys. Rev. Lett., 50(21) 1693, (1983) 
22 G. B. Blanchet, C. R. Fincher, A. J. Heeger, Phys. Rev. Lett. 51(23), 2132 (1983)

23 J. Orenstein, Chapter 36, T. Skotheim Ed.,The Handbook of Conducting Polymers;; (M. Dekker, New York 1986)

24 C. V. Schank, R. Yen, R. L. Fork, J. Orenstein, G. L. Baker, Phys. Rev. Lett. 49(22), 1660 (1982)

25 L. Rothberg, T. M. Jedju, S. Etemad, G. L. Baker, Phys. Rev. Lett. 57(25), 3229 (1986)

26 W. P. Su, J. R. Schrieffer, Proc. Natl. Acad. Sci. 77(10), 5626 (1980)

27 J. Orenstein, Z. Vardeny, G. L. Baker, G. Eagle, S. Etemad, Phys Rev. B30(2), 786 (1984)

28 Z. Verdeny, E. Ehrenfreund, O. Brafman, B. Horovitz, H. Fujimoto, J. Tanaka, M. Tanaka, Phys. Rev. Lett. 57(23), 2995 (1986)

29 Z. Verdeny, E. Ehrenfreund, O. Brafman, Mol. Cryst. Liq. Cryst. 117, 245 (1985)

30 G. B. Blanchet, C. R. Fincher, T. C. Chung, A. J. Heeger, Phys. Rev. Lett. 50(24), 1938 (1983)

31 S. Kivelson, W.-K. Wu, Phys. Rev. B34(8), 5423 (1986)

32 J. D. Flood, A. J. Heeger, Phys. Rev. B28(5), 2356 (1983)

33 J. C. W. Chien, Polyacetylene Chemistry, Physics, and Material Science, (Academic Press: Orlando, 1984) p. 202

34 H. E. Schaffer, R. H. Friend, A. J. Heeger, Phys. Rev. B36(14), 7537 (1987)

35 J. Orenstein, G. L. Baker, Phys. Rev. B49(14), 1043 (1982) 
Appendix 1 Thermodynamic Calculations (all values are in $\mathrm{kcal} / \mathrm{mole}$ )

n-doping

$$
\begin{gathered}
(\mathrm{CH})_{\mathrm{x}}+\left[\mathrm{Na}^{+} \text {Naphthalene }-\right] \rightarrow\left[(\mathrm{CH})_{\mathbf{x}}{ }^{-} \mathrm{Na}^{+}\right]+\text {Naphthalene } \\
\Delta \mathrm{H}=\mathrm{EA}(\text { Naphthalene })^{1}-\mathrm{EA}\left((\mathrm{CH})_{\mathrm{X}}\right)^{2} \\
-10.0=3.5-13.5
\end{gathered}
$$

p-doping

$$
\begin{gathered}
2(\mathrm{CH})_{\mathrm{x}}+3 \mathrm{X}_{2} \rightarrow 2\left[(\mathrm{CH})_{\mathrm{x}}+\mathrm{X}_{3^{-}}\right] \\
2(\mathrm{CH})_{\mathrm{x}}+\mathrm{X}_{2} \rightarrow 2\left[(\mathrm{CH})_{\mathrm{x}^{+}}+\mathrm{X}^{-}\right] \\
2\left\{\mathrm{X}^{-}+\mathrm{X}_{2} \rightarrow \mathrm{X}_{3}^{-}\right\}
\end{gathered}
$$

$$
\Delta \mathrm{H}_{i}=\mathrm{D}_{0}^{4}\left(\mathrm{X}_{2}\right)+2 \mathrm{IE}^{5}\left((\mathrm{CH})_{\mathrm{X}}\right)-2 \mathrm{EA}^{3}(\mathrm{X})+2 \mathrm{~V}(+/-)
$$

$\mathrm{Br}_{2}$

$$
-73.8=45.4+2(184)-2(77.6)+2(-166)
$$

$\mathbf{I}_{2}$

$$
-70.3=35.5+2(184)-2(70.9)+2(-166)
$$

$\Delta \mathrm{H}_{i i}$ (in aqueous solution) ${ }^{6.7}$

$\mathrm{Br}_{2}$

$\mathrm{I}_{2}$

$$
\begin{aligned}
& -3.0=2(-1.5) \\
& -8.4=2(-4.2)
\end{aligned}
$$

$$
\begin{aligned}
\Delta \mathrm{H}_{\mathrm{B}} & =\Delta \mathrm{H}_{i}+\Delta \mathrm{H}_{i i} \\
-76.8 & =-73.8+-3.0 \\
-78.7 & =-70.3+-8.4
\end{aligned}
$$

$\mathrm{Br}_{2}$

$\mathrm{I}_{2}$

(C)

$$
2(\mathrm{CH})_{\mathrm{x}}+4 \mathrm{HX} \rightarrow 2\left[(\mathrm{CH})_{\mathrm{x}}+\mathrm{HX}_{2}^{-}\right]+\mathrm{H}_{2}
$$$$
2\left\{(\mathrm{CH})_{\mathrm{x}}+\mathrm{HX} \rightarrow\left[(\mathrm{CH})_{\mathrm{x}}+\mathrm{X}^{-}\right]+\mathrm{H} \cdot\right\}
$$

$$
\begin{gathered}
2 \mathrm{H}^{-} \rightarrow \mathrm{H}_{2} \\
2\left(\mathrm{X}^{-}+\mathrm{HX} \rightarrow \mathrm{HXX}_{2}^{-}\right)
\end{gathered}
$$

$$
\Delta \mathrm{H}_{i}=2 \mathrm{D}_{0}(\mathrm{HX})+2 \mathrm{IE}\left((\mathrm{CH})_{\mathrm{X}}\right)-2 \mathrm{EA}(\mathrm{X})+2 \mathrm{~V}(+/-)
$$


$\mathrm{HCl}$

$\mathrm{HBr}$

$\mathrm{HCl}$

$\mathrm{HBr}$

$\mathrm{HCl}$

$\mathrm{HBr}$

(D)

$i$
$i i$
$i i i$
$i v$
$v$

$\mathrm{HCl}$

$\mathrm{HBr}$

$\mathrm{HCl}$

$\mathrm{HBr}$

$\mathrm{HCl}$

$$
\Delta \mathrm{H}_{\mathrm{D}}=\Delta \mathrm{H}_{i}+4 \mathrm{Do}(\mathrm{HX})-2 \mathrm{D}_{0}\left(\mathrm{X}_{2}\right)+\Delta \mathrm{H}_{i v}-3 \mathrm{D}_{0}\left(\mathrm{H}_{2}\right)
$$

$\mathrm{HBr}$

$$
73.8=2(102.2)+2(184)-2(83.3)+2(-166)
$$

$$
53.8=2(86.5)+2(184)-2(77.6)+2(-166)
$$

$\Delta \mathrm{H}_{i i i}$ (in Tetrahydrothiophene 1,1 dioxide) ${ }^{8}$

$$
\begin{aligned}
-16.2 & =2(-8.1) \\
-9.8 & =2(-4.9)
\end{aligned}
$$

$$
\begin{gathered}
\Delta \mathrm{H}_{\mathrm{C}}=\Delta \mathrm{H}_{i}-\mathrm{D}_{\mathrm{O}}\left(\mathrm{H}_{2}\right)+\Delta \mathrm{H}_{i i i} \\
-45.6=73.8+-103.2+-16.2 \\
-59.4=53.6+-103.2+-9.8
\end{gathered}
$$

$2(\mathrm{CH})_{x}+6 \mathrm{HX} \rightarrow 2\left[(\mathrm{CH})_{x}+\mathrm{X}_{3}^{-}\right]+3 \mathrm{H}_{2}$

$$
\begin{gathered}
2\left\{(\mathrm{CH})_{\mathrm{x}}+\mathrm{HX} \rightarrow\left[(\mathrm{CH})_{\mathrm{x}}+\mathrm{X}^{-}\right]+\mathrm{H} \cdot\right\} \\
4\{\mathrm{HX} \rightarrow \mathrm{X} \cdot+\mathrm{H} \cdot\} \\
2\left\{2 \mathrm{X} \cdot \rightarrow \mathrm{X}_{2}\right\} \\
2\left\{\mathrm{X}_{2}+\mathrm{X}^{-} \rightarrow \mathrm{X}_{3}{ }^{-}\right\} \\
3\left\{2 \mathrm{H} \cdot \rightarrow \mathrm{H}_{2}\right\}
\end{gathered}
$$

$$
\Delta \mathrm{H}_{\mathrm{i}}=2 \mathrm{D}_{\mathrm{o}}(\mathrm{HX})+2 \mathrm{IE}\left((\mathrm{CH})_{\mathrm{x}}\right)-2 \mathrm{EA}(\mathrm{X})+2 \mathrm{~V}(+/-)
$$

$$
73.8=2(102.2)+2(184)-2(83.3)+2(-166)
$$

$$
53.8=2(86.5)+2(184)-2(77.6)+2(-166)
$$

$$
\Delta \mathrm{H}_{i v} \text { (in aqueous solution) }
$$

$$
\begin{aligned}
<14 & =73.8+4(102.2)-2(79.5)+- \text { small }-3(103.2) \\
-1 & =53.6+4(86.5)-2(45.5)+-3.0-3(103.2)
\end{aligned}
$$

Chemical Stabilization of Photoinduced Charged Defects

$$
2 S^{-}+2 S^{+}+4 H X \rightarrow 2 S_{0}+2\left[S^{+} \mathrm{HX}_{2}^{-}\right]+\mathrm{H}_{2}
$$


$2\left[\mathrm{~S}^{-}+\mathrm{HX} \rightarrow \mathrm{S}_{0}+\mathrm{X}^{-}+\mathrm{H}^{-}\right\}$

$\ddot{u}$

$2 \mathrm{H} \cdot \rightarrow \mathrm{H}_{2}$

iii

iv

$$
\begin{gathered}
2\left\{\mathrm{X}^{-}+\mathrm{HX} \rightarrow \mathrm{HXX}_{2}^{-}\right\} \\
2\left\{\left[\mathrm{~S}_{\mathrm{O}} \mathrm{HX}_{2}^{-}\right]+\mathrm{S}^{+} \rightarrow\left[\mathrm{S}^{+} \mathrm{HX}_{2}^{-}\right]+\mathrm{S}_{\mathrm{o}}\right\}
\end{gathered}
$$

$$
\Delta \mathrm{H} i=2 \mathrm{D}_{0}(\mathrm{HX})-2 \mathrm{EA}(\mathrm{X})+2 \mathrm{EA}\left(\mathrm{S}^{\circ}\right)
$$

$\mathrm{HCl}$

$$
44.4=2(102.2)-2(83.3)+2(3.3)
$$

$\mathrm{HBr}$

$$
24.4=2(86.5)-2(77.6)+2(3.3)
$$

$$
\Delta \mathrm{H}_{\mathrm{E}}=\Delta \mathrm{H}_{i}-\mathrm{D}_{\mathrm{o}}\left(\mathrm{H}_{2}\right)+\Delta \mathrm{H}_{i i i}(\mathrm{C})+2 \mathrm{~V}(+/-)
$$

$\mathrm{HCl}$

$$
-407.0=44.4-103.2+-16.2-2(166)
$$

$\mathrm{HBr}$

$$
-420.6=24.4-103.2+-9.8-2(166)
$$

$$
2 \mathrm{P}^{-}+2 \mathrm{P}^{+}+4 \mathrm{HX} \rightarrow 2\left[\mathrm{P}^{+} \mathrm{HX}_{2}^{-}\right]+\mathrm{H}_{2}+2(\mathrm{CH})_{\mathrm{X}}
$$

$$
2\left\{\mathrm{P}^{-}+\mathrm{HX} \rightarrow(\mathrm{CH})_{\mathrm{X}}+\mathrm{X}^{-}+\mathrm{H}^{-}\right\}
$$

$$
2 \mathrm{H} \cdot \rightarrow \mathrm{H}_{2}
$$$$
2\left\{\mathrm{X}^{-}+\mathrm{HX} \rightarrow \mathrm{HX}_{2}^{-}\right\}
$$$$
2\left\{\mathrm{HX}_{2}^{-}+\mathrm{P}^{+} \rightarrow\left[\mathrm{P}^{+} \mathrm{HX}_{2}^{-}\right]\right\}
$$

$$
\Delta \mathrm{H}_{i}=2 \mathrm{D}_{0}(\mathrm{HX})-2 \mathrm{EA}(\mathrm{X})+2 \mathrm{EA}\left((\mathrm{CH})_{\mathrm{x}}\right)
$$

$\mathrm{HCl}$

$$
64.8=2(102.2)-2(83.3)+2(13.5)
$$

$\mathrm{HBr}$

$$
44.8=2(86.5)-2(77.6)+2(13.5)
$$

$$
\Delta \mathrm{H}_{\mathrm{F}}=\Delta \mathrm{F}_{i}-\mathrm{D}_{\mathrm{c}}\left(\mathrm{H}_{2}\right)+\Delta \mathrm{H}_{i i i}(\mathrm{C})+2 \mathrm{~V}(+/-)
$$

$\mathrm{HCl}$

$$
-386.6=64.8-103.2+-16.2-2(166)
$$

$\mathrm{HBr}$

$$
-400.2=44.8-103.2+-9.8-2(166)
$$

l W. E. Wentworth, W. Ristan, J. Phys. Chem.73, 2126 (1969)

2 W. E. Wentworth, E. Chen, J. E. Lovelock, J. Phys. Chem.70, 445 (1966)

3 C. E. Moore, Atomic Energy Levels, vol. III, (Circular of the National Bureau of Standards 467,1958 )

4 G. Herzberg, Molecular Spectra and Molecular Structure I. Specta of Diaromic Molecules, (Van Nostrand Reinhold Bo. 1950) 
5 K. Watanabe, T. Nakayama, J. Mottl. J. Quant. Spectroscopy and Energy Transfer, 2, 369 (1962)

6 R. W. Ramette, D. A. Palmer, J. Soln. Chem., 15(5), 387 (1986)

7 D. A. Palmer, R. W. Ramette, R. E. Mesmer, J. Soln. Chem., 13(9), 673 (1984)

8 R. L. Beniot, M. Rinfret, R. Domain, Inorg. Chem., 11, 2603 (1972) 


\section{Appendix 2 Kinetic Calculations}

Predicted relative rates for the chemical doping and stablization of photoinduced defects reactions at 50 and $30 \mathrm{~K}$. Where $A_{p}=2.5 \times 10^{-5} A_{s}$

\begin{tabular}{|c|c|c|c|}
\hline & $\begin{array}{c}\text { Chemical } \\
\mathrm{E}_{\mathrm{a}} \\
\text { (kcal/mole) }\end{array}$ & $\begin{array}{c}\text { Soliton Stablization } \\
\mathrm{E}_{\mathrm{a}} \mathrm{s}= \\
\mathrm{Ea}\left(\Delta \mathrm{H}_{i}(\mathrm{E}) / \Delta \mathrm{H}_{i}(\mathrm{C})\right)\end{array}$ & $\begin{array}{c}\text { Polaron Stablization } \\
E_{a} \mathrm{p}= \\
\mathrm{Ea}\left(\Delta \mathrm{H}_{i}(\mathrm{~F}) / \Delta \mathrm{H}_{i}(\mathrm{C})\right)\end{array}$ \\
\hline \multirow{2}{*}{ Temperature } & $A_{c} \times \exp \left(-E_{a} / R T\right)$ & $A_{p} \times \exp \left(-E_{a} / R T\right)$ & $A_{p} \times \exp \left(-E_{a} / R T\right)$ \\
\hline & $A_{c} \times \exp \left(-E_{a} / R 60\right)$ & $A_{c} \times \exp \left(-E_{a} / R 60\right)$ & $A_{c} \times \exp \left(-E_{a} / R 60\right)$ \\
\hline $\mathrm{E}_{\mathrm{a}}(\mathrm{kcal} / \mathrm{mole})=$ & 3 & 1.8 & 2.6 \\
\hline $50 \mathrm{~K}$ & 1 & 4.1 & $1.4 \times 10^{-3}$ \\
\hline $30 \mathrm{~K}$ & $2.1 \times 10^{-9}$ & $2.5 \times 10^{-5}$ & $4.0 \times 10^{-11}$ \\
\hline $\mathrm{E}_{\mathrm{a}}(\mathrm{kcal} / \mathrm{mole})=$ & 6 & 3.6 & 5.3 \\
\hline $50 \mathrm{~K}$ & 1 & $6.6 \times 10^{5}$ & $2.7 \times 10^{-2}$ \\
\hline $30 \mathrm{~K}$ & $4.2 \times 10^{-18}$ & $2.5 \times 10^{-5}$ & $1.2 \times 10^{-17}$ \\
\hline $\mathrm{E}_{\mathrm{a}}(\mathrm{kcal} / \mathrm{mole})=$ & 9 & 5.4 & 7.9 \\
\hline $50 \mathrm{~K}$ & 1 & $1.1 \times 10^{11}$ & 1.5 \\
\hline $30 \mathrm{~K}$ & $7.8 \times 10^{-27}$ & $2.5 \times 10^{-5}$ & $2.0 \times 10^{-23}$ \\
\hline $\mathrm{E}_{\mathrm{a}}(\mathrm{kcal} / \mathrm{mole})=$ & 12 & 7.2 & 10.5 \\
\hline $50 \mathrm{~K}$ & 1 & $1.7 \times 10^{16}$ & $8.2 \times 10^{10}$ \\
\hline $30 \mathrm{~K}$ & $1.8 \times 10^{-35}$ & $2.5 \times 10^{-5}$ & $3.2 \times 10^{-29}$ \\
\hline
\end{tabular}

